\title{
Synthesis and stability evaluation of novel peptidomimetic Caspase-1 inhibitors for topical application
}

\author{
Sandrine Chambon ${ }^{a}$, Sandrine Talano a, Corinne Millois a , Laurence Dumais a , \\ Romain Pierre ${ }^{a}$, Loic Tomas ${ }^{a}$, Céline Mathieu ${ }^{a}$, Anne-Laurence Ghilini ${ }^{a}$, \\ Nicolas Vanthuyne ${ }^{\mathrm{b}}$, Kevin Reverse ${ }^{\mathrm{a}}$, Anne Brethon ${ }^{\mathrm{c}}$, Vincent Rodeschini ${ }^{\mathrm{c}}$, \\ Catherine Comino ${ }^{a}$, Grégoire Mouis ${ }^{a}$, Ghizlane El-Bazbouz ${ }^{a}$, Laurence Clary ${ }^{a}$, \\ Jean-François Fournier ${ }^{\text {a }}$, Claire Bouix-Peter ${ }^{a}$, Craig S. Harris ${ }^{\text {a, }}{ }^{*}$, Laurent F. Hennequin ${ }^{a}$ \\ a Nestlé Skin Health R\&D, 2400 Route de Colles, 06410 Biot, France \\ ${ }^{\mathrm{b}}$ Aix-Marseille Univ., CNRS, Centrale Marseille, iSm2, Marseille, France \\ ${ }^{c}$ Edelris, 115 Avenue Lacassagne, 69003 Lyon, France
}

\begin{abstract}
A B S T R A C T
During our search for topically-active Caspase-1 inhibitors, we identified a novel class of potent inhibitors based on a 1,3,5-trisubstituted uracil motif equipped with an L-aspartate semi-aldehyde derived warhead. In the literature, the majority of Caspase-1 inhibitors possessing the same warhead have been designed and evaluated for oral administration as the ethyl acetal pro-drug form. For our topical program, the pro-drug acetal form was not fully hydrolysed in the skin and was unstable in many of our standard topical excipients, therefore, we were obliged to focus on the actual hemiacetal drug form of the molecule during our drug discovery program. Our work focuses on both the synthesis and achiral and chiral stability of the final drug molecules in topical excipients.
\end{abstract}

\section{Introduction}

Caspases are a family of protease enzymes called Cysteine Aspartic Proteinases. There are 12 known Caspases in humans. Caspases 1, 4, 5, 11 and 12 are all inflammatory caspases and 2, 3, 6, 7, 8, 9 and 10 are all associated with apoptosis [1]. All caspase substrates are cleaved from the C-terminus of an aspartic acid residue $\mathrm{C}$-terminal in the $\mathrm{P} 1$ region of the binding site. Interleukin$1 \beta$ converting enzyme (ICE), also known as Caspase- 1 , is the principal enzyme responsible for cleavage and activation of pro-Interleukin-1 $\beta$ (pro-IL-1 $\beta$ ) to its active form IL-1 $\beta$, which in turn is involved in the pathogenesis of several inflammatory disorders including acne vulgaris [2]. The cleavage of pro-IL- $1 \beta$ to IL- $1 \beta$ occurs through deprotonation of a cysteine residue via a neighbouring histidine. Cytokine IL-1 $\beta$ plays an important role in local and

\footnotetext{
* Corresponding author.

E-mail address: craig.harris@galderma.com (C.S. Harris).
}

systemic inflammation and has been reported to target numerous cells involved in inflammatory acne [3]. In a recent study, we reported a comparison of lesional versus non-lesional biopsies of patients with inflammatory acne and through transcriptomic and proteomic studies, we showed a strong induction of IL-1 $\beta$ mRNA and IL-1 $\beta$ protein in lesional biopsies [4]. Consequently, the treatment of inflammatory acne with a topical agent targeting Caspase1 presented an exciting and novel opportunity for the treatment of moderate to severe acne.

Medicinal chemists have developed potent inhibitors of the catalytic site principally by using masked aldehydes [5]. These form a reversible covalent linkage with the cysteine residue to block the enzyme from functioning. In 1992, Thornberry et al., inspired from the actual sequence of Caspase I substrate pro-IL-1 $\beta$, discovered a tetrapeptide motif, Ac-YVAD-CHO with nanomolar potency against Caspase I. The C-terminus of the aspartic acid residue was modified to an aldehyde resulting in reversible covalent inhibition of the enzyme through a hemithioacetal linkage (Fig. 1). 


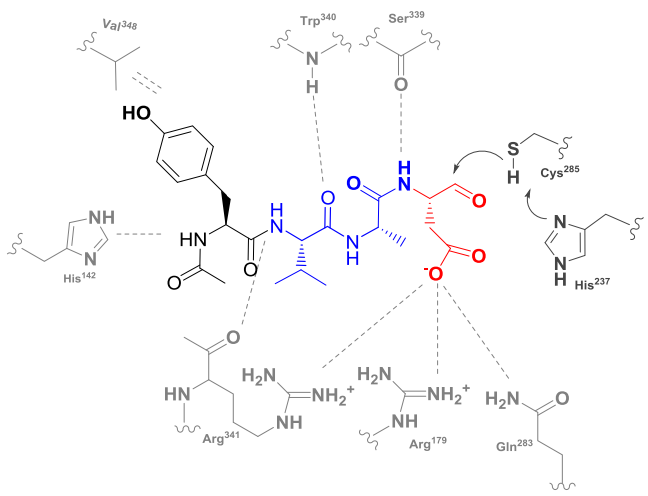

Fig. 1. Binding mode of Ac-YVAD-CHO in Caspase I (Black, P4 pocket; blue P3/P2 region; red, P1 warhead region).

To overcome the poor ADMET of the early peptidic leads, potent peptidomimetic inhibitors of Capsase-1 [6], Pralnacasan (VX740) and Belnacasan (VX765) were developed and have been progressed to clinical studies for the treatment of rheumatoid arthritis and treatment-resistant epilepsy. Inspired by their approach, we set about designing our own cyclic peptidomimetic inhibitors based on scaffolds while preserving the 3 point $\mathrm{H}$-bonding network and the aspartaldehyde warhead (Fig. 2) [7].

\section{Results and discussion}

We developed several synthetic strategies to deliver the piperidin-2,6-dione and pyrrolidin-2,5-dione based inhibitors that we have already detailed in a recent communication [8], focusing principally on: a) P1 warhead exploration; and b) probing the P4 region both exemplified in Schemes 1 and 2, respectively.

For topical medicinal chemistry, activity is always an important driver but the solution stability of our actives is another major consideration as the API is not formulated in solid form but in wet form such as creams, lotions or gels [9]. The topical excipient selected for screening purposes was a $96 \% \mathrm{w} / \mathrm{w}$ aqueous ethanol. Solutions at $0.01 \%(\mathrm{w} / \mathrm{w})$ were monitored by HPLC during 3 months. Ideally, we would expect our developable candidate to have $>90 \%$ of its original peak area remaining after 3 months at $40^{\circ} \mathrm{C}$. This can be extrapolated to a shelf-life over $>1 \mathrm{yr}$ in the final topical formulation at room temperature [10].

Early on in our investigations, we established that the ethyl acetal pro-drug form (eg., 8) of our final compounds was actually less stable than the hemiacetal drug form (eg., $\mathbf{1}$ and $\mathbf{2}$ ) in all of our topical excipients. Moreover, the pro-drug was also not cleaved sufficiently quickly enough to the drug form in our in vitro and

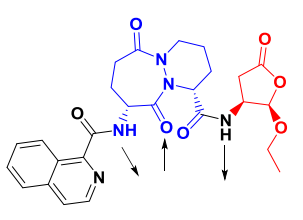

Pralnacasan (VX-740)

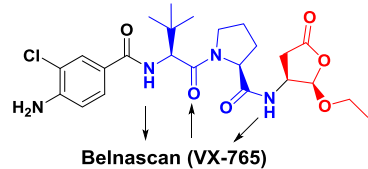

Belnascan (VX-765)

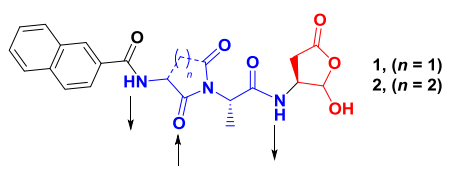

Fig. 2. Our design rationale for a new classes of imide-based peptidomimetic Caspase1 inhibitors.

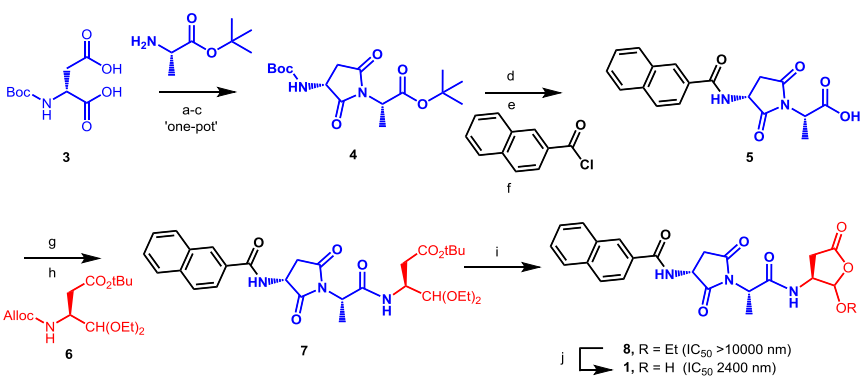

Scheme 1. A representative route designed for P1 exploration and used to deliver eg., 1. Reagents and conditions: (a) EDCI, DCM, 16 h, r.t.; (b) H-Ala-OtBu. $\mathrm{HCl}$, DIPEA, 2 h, r.t.; (c) EDCl, HOBt (1.5 eq.), 16 h, r.t., $58 \%$; (d) $4 \mathrm{M} \mathrm{HCl}$ in dioxane, EtOAc, $5^{\circ} \mathrm{C}, 16$ h, quant.; (e) 2-naphthoyl chloride, DIPEA, THF, $0^{\circ} \mathrm{C}$-r.t., $4 \mathrm{~h}$, 59\%; (f) TFA-DCM (1:3), r.t., 6 h, quant. (g) $\mathrm{Pd}\left(\mathrm{PPh}_{3}\right)_{4}, \mathrm{DMBA}, \mathrm{DCM}$, r.t., $15 \mathrm{~min}$; (h) 5 pre-activated EDCI, HOBt, DCM, DMF, $0^{\circ} \mathrm{C}, 15 \mathrm{~min}$, amine from step (g) added, r.t., 16 h, 72\%; (i) TFA, DCM, r.t., 59\%; (j) $2 \mathrm{~N} \mathrm{HCl}$ (aq), $\mathrm{MeCN}, 0^{\circ} \mathrm{C}$-r.t., $32 \%$.

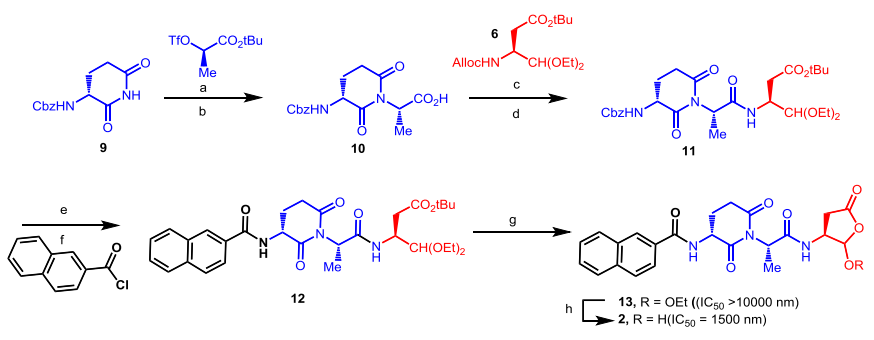

Scheme 2. A representative route designed for $\mathrm{P} 4$ exploration and used to deliver eg., 2. Reagents and conditions: (a) $\mathrm{K}_{2} \mathrm{CO}_{3}$, MeCN, r.t., $53 \%$; (b) TFA, $0{ }^{\circ} \mathrm{C}$-r.t., quant.; (c) $\mathrm{Pd}\left(\mathrm{PPh}_{3}\right)_{4}, \mathrm{DMBA}, \mathrm{DCM}$, r.t., 15 min; (d) 10 pre-activated EDCI, HOBt, DCM, DMF, $0^{\circ} \mathrm{C}$, 15 min, amine from step (c) added, r.t., 16 h, $62 \%$; (e) $\mathrm{H}_{2}, \mathrm{Pd}(\mathrm{OH})_{2}-\mathrm{C}$, EtOAc, r.t., 78\%; (f) TEA, DCM or 43\%; (g) TFA, DCM, r.t., $75 \%$; (h) $2 \mathrm{~N} \mathrm{HCl} \mathrm{(aq),} \mathrm{MeCN}, 0^{\circ} \mathrm{C}$-r.t., $52 \%$.

in vivo assays. Therefore, we targeted the preparation of the drug form of our final compounds for evaluation in our testing cascade.

Both pyrrolidin-2,5-dione and piperin-2,6-dione scaffolds 1 and 2 provided our first lead compounds with moderate activity $2400 \mathrm{nM}$ and $1500 \mathrm{nM}$, respectively, against Caspase-1 (Schemes 1 and 2). However, the stability studies were very disappointing with all of our early designs. Both drug $(\mathbf{1}, \mathbf{2})$ and pro-drug $(\mathbf{8})$ did not achieve an acceptable level of stability ( $>90 \%)$ at $40^{\circ} \mathrm{C}$ after just $30 \mathrm{~d}$ and only 2 had acceptable stability with just $9 \%$ degradation at $23^{\circ} \mathrm{C}$ after 30 days (Fig. 2). It is also interesting to note that the drug form 1 was more stable at $40^{\circ} \mathrm{C}$ than the pro-drug form 8 with $70 \%$ versus $58 \%$ of the original peak remaining, respectively (Fig. 3 ).

By HRMS, we managed to identify the principal degradation products of our lead piperidin-2,6-dione series being due to ringopening and loss of the warhead, not through hydrolysis of the amide bond but through tautomerisation followed by alcoholysis of

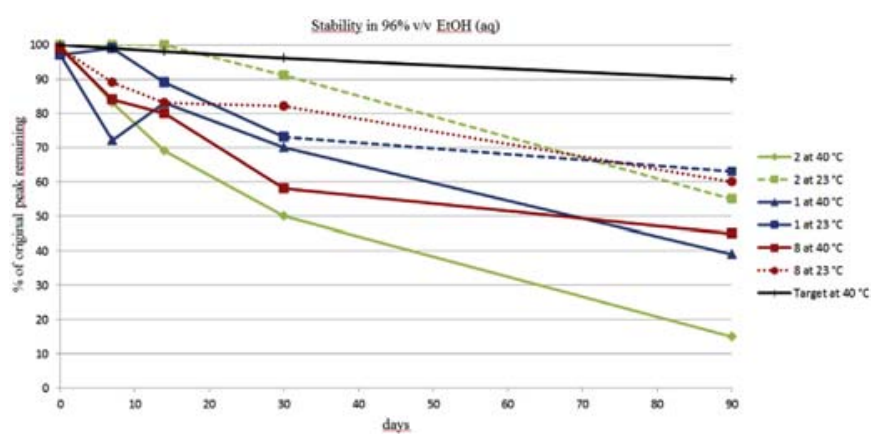

Fig. 3. Comparative stability evaluation of $\mathbf{1}, \mathbf{2}$ and $\mathbf{8}$. 
the intermediate enamine or by a retro-Michael reaction (Fig. 4).

To overcome this stability issue of the piperidin-2,6-dione series, we decided to transpose our SAR to a 1,3,5-trisubstituted uracil scaffold [7,11]. We anticipated that the stability issues would be addressed by preventing ring opening and replacing the sensitive chiral center at $\mathrm{C} 3-\mathrm{H}$ of the piperidin-2,6-dione unit that we had great difficulty in controlling during the assembly of our final products (eg., 1 and 14) [8]. The first match pair (14 v 15), incorporating Sunesis' quinoxaline P4 fragment [12], showed an impressive $\sim 40$ fold gain in potency and solution stability improved significantly, $55 \%$ v $84 \%$, respectively. However, during the assembly of our final molecules we encountered great difficulty to control the stereogenic center off $\mathrm{N}-3$, finishing routinely with a racemate. To overcome this epimerization issue, we introduced a cyclopropyl group [13]. Indeed, as discussed previously by us [7], we suspected that it should be accommodated in the limited space in the binding cleft between $\mathrm{Val}^{338}$ and $\operatorname{Trp}^{340}$. Moreover, after further SAR (structure activity relationship) and SPR (structure property relationship) investigation, we identified $\mathbf{1 6}$ as our new lead candidate with much improved potency, stability, photosafety and synthetic feasibility (Fig. 5) [7].

The key step for our uracil fragments relied on a novel $3 \mathrm{MCR}$ (multi-component reaction) construction of the 5-nitrouracil fragment (21) with a cyclopropyl moiety at $N-1$ described in a recent letter [13]. From 21, alkylation at $N$-3 followed by reduction using dissolving metal conditions [14] afforded the amino P2/P3 fragment 23, coupling with the optimized P4 fragment (24) using COMU ((1-cyano-2-ethoxy-2-oxoethylidenaminooxy)dimethylamino-morpholino-carbenium hexafluorophosphate) [15], followed by Alloc deprotection afforded $\mathbf{2 5}$. The synthesis terminated with installation of the precyclised P1 warhead fragment 26

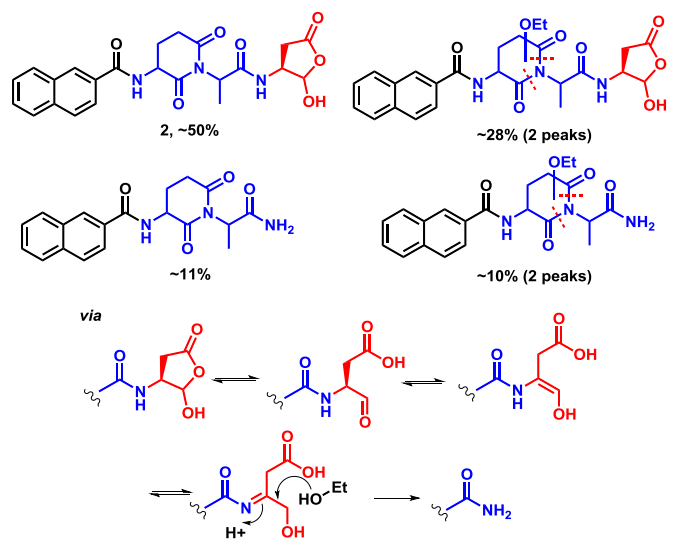

Fig. 4. Major product distribution of 2 determined by LC-HRMS in $96 \% \mathrm{w} / \mathrm{w} \mathrm{EtOH}(\mathrm{aq})$ after heating at $40^{\circ} \mathrm{C}$ for 30 days.

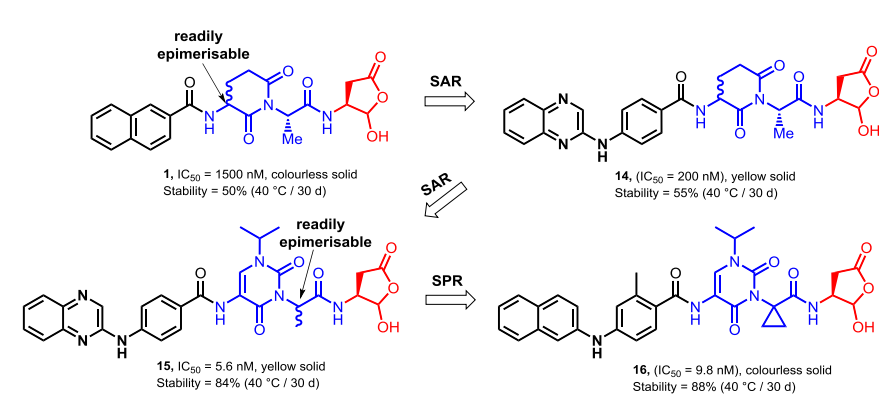

Fig. 5. A simplified SAR and SPR evolution of the early lead based on a piperidin-2,6dione P2/P3 peptidomimetic scaffold to the optimized 1,3,5-trisubstituted uracil scaffold. through a one-pot Alloc deprotection/amide coupling step followed by hydrolysis of the pro-drug ethyl acetal $\mathbf{2 7}$ to afford hemiacetal 16 (Scheme 3).

Our new lead compound $\mathbf{1 6}$ was not only more than 150 times more potent but also much more stable than our early designs $\mathbf{1}$ and 2 (Fig. 6) [7]. After $30 \mathrm{~d}$ at $40^{\circ} \mathrm{C}$, only $17 \%$ degradation was observed with compared to $50 \%$ with our first lead $\mathbf{1}$. Although we had not achieved $<10 \%$ degradation after 3 months at $40^{\circ} \mathrm{C}$, we were confident that we could achieve our target value by exploring other topical excipients. As illustrated previously (Fig. 3), the prodrug form (27) was also less stable than the drug form (16) with $60 \%$ versus $40 \%$ degradation observed after 3 months at $40^{\circ} \mathrm{C}$, respectively.

As we moved further into lead optimisation, we prepared the enantiomer (28) of our lead compound (16) to test its activity and use as a standards to monitor potential chiral degradation (epimerization at C-3) of the P1 warhead fragment. Although the furanone warhead of compound $\mathbf{1 6}$ has two chiral centers, the $\mathrm{C}-2-\mathrm{OH}$ epimers are rapidly interconverting through ring opening and closing of 16-aldehyde. Consequently, at best, immediately after

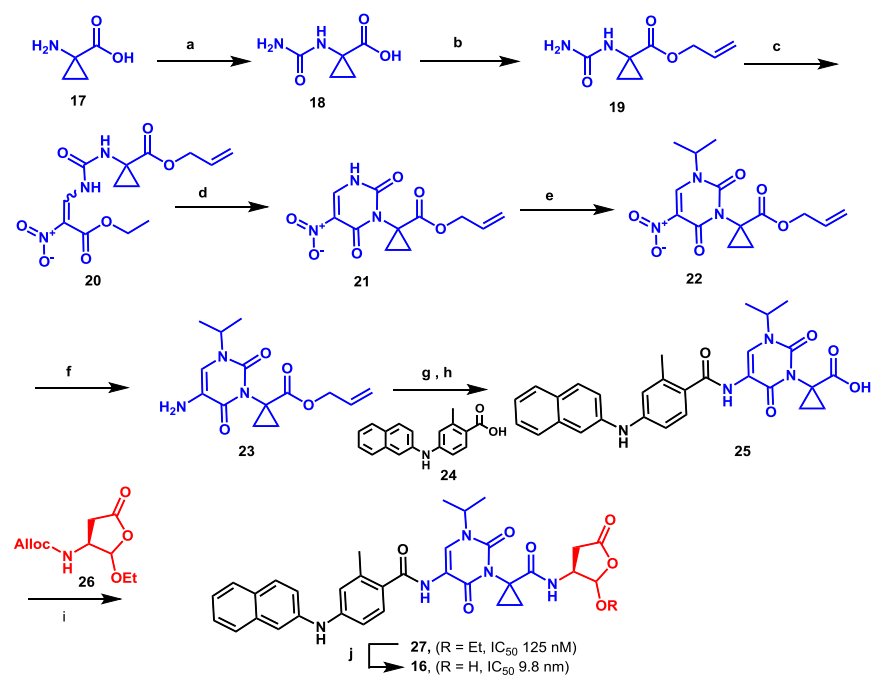

Scheme 3. Principal route employed for the preparation $\mathbf{1 6}$ and its analogues. Reagents and conditions: (a) KOCN, water, reflux, yield 78\%; (b) Allyl bromide, DIPEA, $\mathrm{THF} / \mathrm{H}_{2} \mathrm{O}(10: 1), 60^{\circ} \mathrm{C}, 18 \mathrm{~h}$, yield $68 \%$; (c) ethyl nitroacetate, triethyl orthoformate, toluene, reflux, yield 79\%; (d) $\mathrm{Cs}_{2} \mathrm{CO}_{3}, \mathrm{MeCN}$, reflux, 78\%; (e) 2-iodopropane, $\mathrm{K}_{2} \mathrm{CO}_{3}$, DMF, r.t., $24 \mathrm{~h}, 46 \%$; (f) $\mathrm{Fe}(\mathrm{s}), \mathrm{NH}_{4} \mathrm{Cl}$ (aq), THF, EtOH- ${ }_{2} \mathrm{O}, 70^{\circ} \mathrm{C}, 1 \mathrm{~h}, 98 \%$; (g) 24, COMU, NMM, DMF, $50{ }^{\circ} \mathrm{C}, 48 \mathrm{~h}, 90 \%$; (h) $\mathrm{Pd}\left(\mathrm{PPh}_{3}\right)_{4}, \mathrm{DMBA}, \mathrm{DCM}$, r.t., $45 \mathrm{~min}, 60 \%$; (i) $i$. deprotection of 26, $\mathrm{Pd}\left(\mathrm{PPh}_{3}\right)_{4}, \mathrm{DMBA}, \mathrm{DCM}$, r.t., $15 \mathrm{~min}$; ii. pre-activation of 25 with HATU, DIPEA, DCM-DMF, r.t., $16 \mathrm{~h}$; iii. products from step $i$ added dropwise to ii and reaction mixture stirred at r.t., 16 h, quantitative; (j) $2 \mathrm{M} \mathrm{HCl}$ (aq), $\mathrm{MeCN}$, r.t., 2 h, $65 \%$.

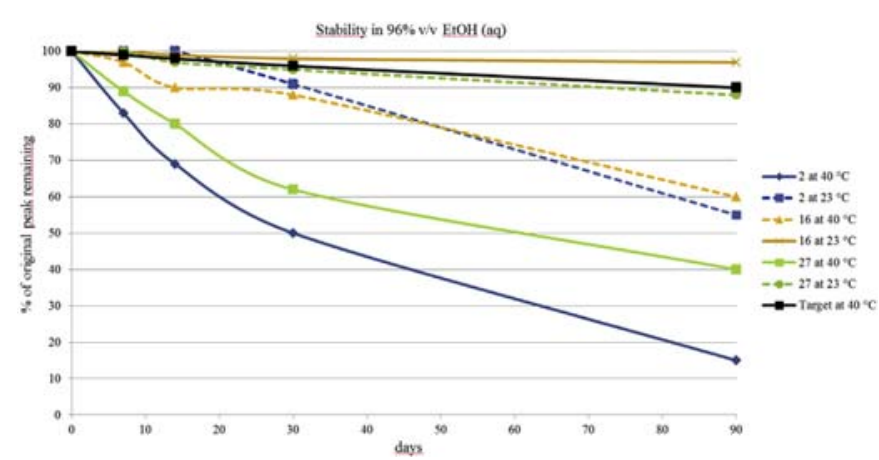

Fig. 6. Comparative stability evaluation of $\mathbf{1 6}$ versus its prodrug $\mathbf{2 5}$ and early piperidin-2,6-dione lead 2. 
the hydrolysis of the pro-drug, we obtain $\mathbf{1 6}$ as a $1: 1$ mixture of diastereomers $(3 S, 2 R S)$, racemic at the hydroxyl-bearing carbon C2 (Scheme 4).

Both 16 and 28 were prepared separately and assayed. They were equipotent which was surprising looking at what had been reported in the literature, raising the question of whether epimerization at C-3 was occurring during hydrolysis of the pro-drug or during storage in DMSO solution. We confirmed that epimerization was not occurring at the hydrolysis stage either by reduction of the aldehyde $\mathbf{1 6}$ to the alcohol $\mathbf{2 9}$ or by condensation to the benzyloxime 30 immediately after isolation. Therefore, epimerization must be occurring during dissolution and storage of $\mathbf{1 6}$ in DMSO or during the in vitro assay (Scheme 5).

Next, we decided to study the chiral stability of $\mathbf{1 6}$ in our model excipient (96\% EtOH (aq)). While we could have employed the derivatisation methods shown in Fig. 7 to monitor chiral degradation at selected time points, we wanted to eliminate all possible contributory factors and focused on the development of a chiral chromatographic method.

The separation of racemic $\mathbf{1 6}$ was tested by a preliminary screening of 8 chiral columns (Chiralpak IA, IB, IC, ID, IE, IF, Whelk-O1 and Ulmo) with a mixture heptane/ethanol/chloroform (2/4/4) as the mobile phase. Chiralpak IC, cellulose tris(3,5-dichlorophenylcarbamate) polymer immobilized on silica, proved to be able to partially separate the enantiomers as shown on the reported UV chromatogram (Fig. 7a). The analytical conditions on this column were then optimized. The use of less polar eluent gave broad peaks (Fig. 7b), heptane/ ethanol binary mixtures improved the enantioselectivity but with fronting of the 2 peaks (Fig. 7c), separation was lost with heptane/2propanol mixtures and enantioselectivity decreased rapidly as temperature was increased. Finally, addition of $0.5 \%$ of triethylamine in the mobile phase allowed a baseline separation of the enantiomers with an enantioselectivity of 1.9 and a resolution of 1.8 (Fig. 7e). We retained these conditions to follow the chiral degradation of our final products.

The appearance of an additional peak in the presence of TFA (Fig. 7d) was interesting and prompted us to invest more time in

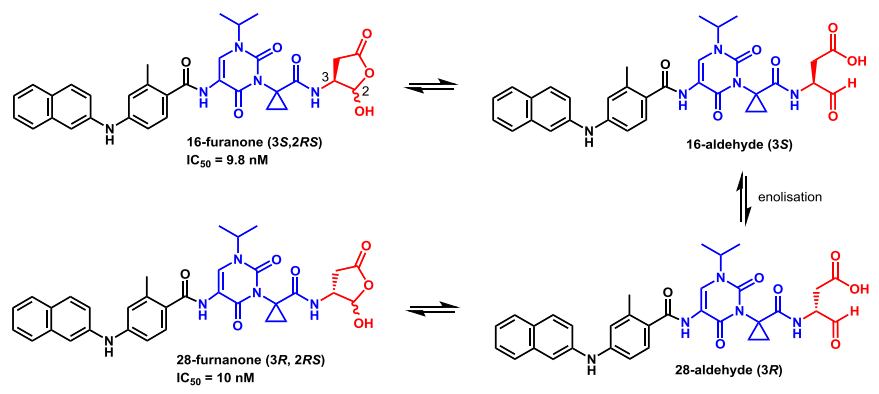

Scheme 4. Proposed mechanism of epimerization of $\mathbf{1 6}$.

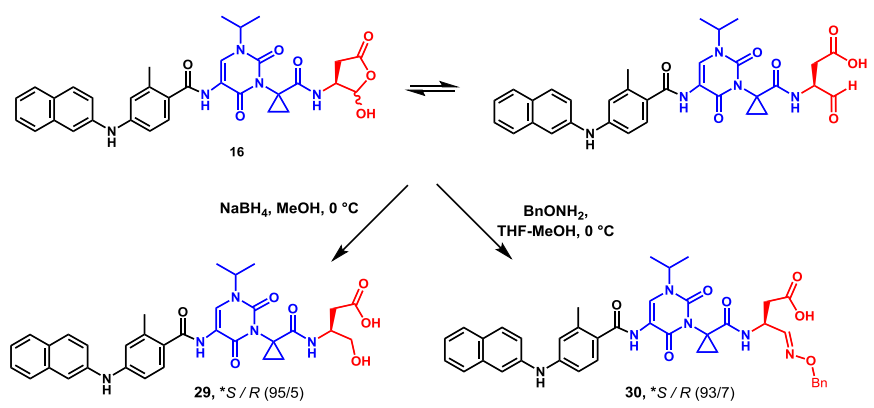

Scheme 5. Methods developed to test for epimerization during hydrolysis step.
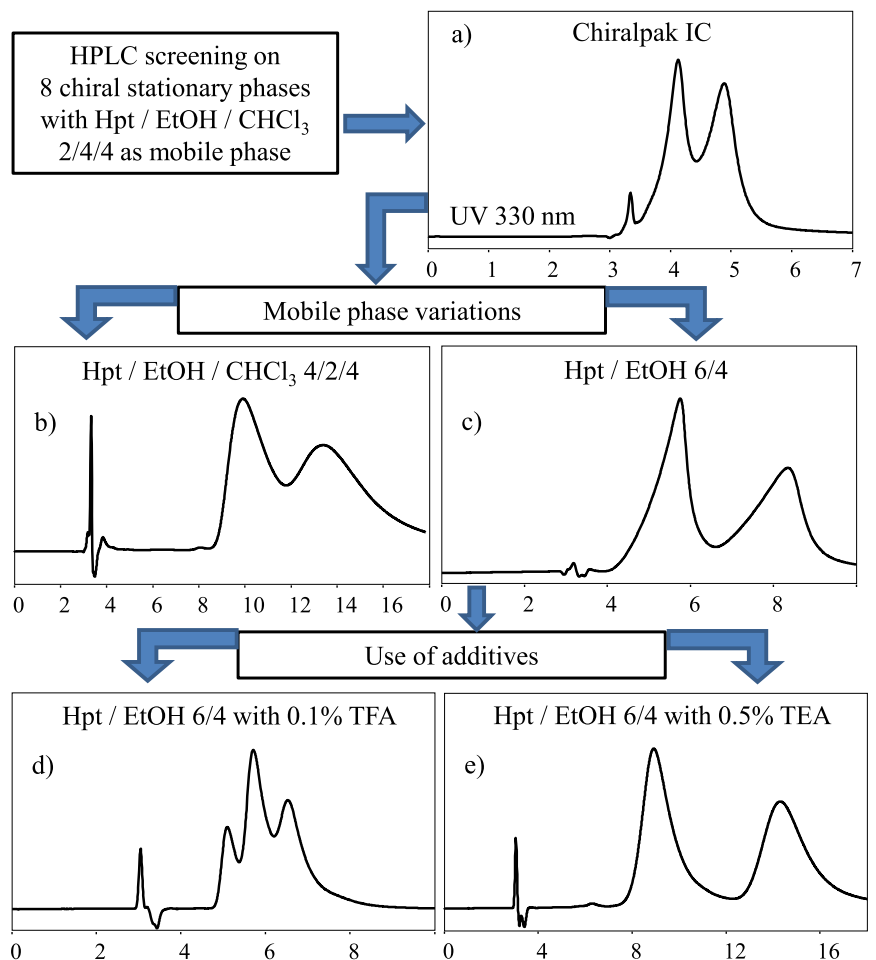

Fig. 7. Chiral HPLC method development for the separation of the enantiomer of $\mathbf{1 6}$ Chiralpak IC, $1 \mathrm{~mL} / \mathrm{min}, 25^{\circ} \mathrm{C}$, detection UV at $330 \mathrm{~nm}$.

method development. We hypothesized that under this condition, the extra peak arose from the start of the separation of the diastereomeric warheads (ie., in the case of $16,3 \mathrm{~S} / 2 \mathrm{~S}$ from $3 \mathrm{~S} / 2 \mathrm{R}$ ). Further screening of different columns and a new optimisation of the analytical conditions highlighted an exchange between different species, as indicated by the shape of the chromatograms (Fig. 8). In this acidic condition, at $40^{\circ} \mathrm{C}$, the opened aldehyde forms are in equilibrium with the hydroxy-furanone ring forms and all 4 diastereomers could be distinguished along with later running peaks that we assumed to be the open aldehyde forms assigned on the basis of mass spectrometry alone. These observations confirmed the configurational stability of the carbon bearing the nitrogen atom and the fast epimerization of the carbon bearing the hydroxyl group.

With our optimized chiral chromatographic conditions in hand (Fig. 7e), we monitored the chiral degradation of $\mathbf{1 6}$ in different excipients. Routinely, in the lead optimisation phase of our drug discovery programs, we would be expecting to achieve an e.r. of

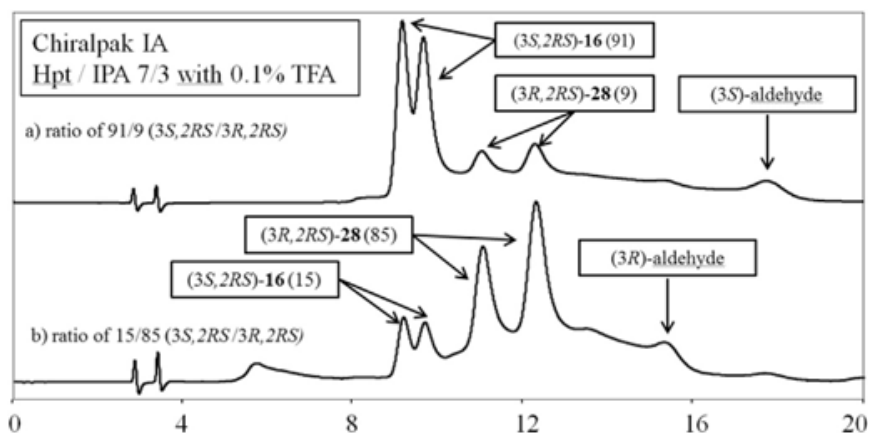

Fig. 8. Equilibria between opened and closed warhead forms of $\mathbf{1 6} \& \mathbf{2 8}$ evidenced by chromatographic profile on Chiralpak IA, $1 \mathrm{~mL} / \mathrm{min}, 40^{\circ} \mathrm{C}$, detection UV at $330 \mathrm{~nm}$. 
$\geq 95: 5$. As attested by the results in Table 1 , racemisation was practically complete at $40^{\circ} \mathrm{C}$ after just $1 \mathrm{~d}$ in $96 \% \mathrm{v} / \mathrm{v}$ EtOH (aq). Even after $30 \mathrm{~d}$ of storage at $0{ }^{\circ} \mathrm{C}$, an unacceptable e.r. of $89 / 11$ was obtained (entry 1 ). Similar results were also obtained as a $0.1 \% \mathrm{w} / \mathrm{w}$ solution in other commonly employed topical excipients from our screening panel. In summary, with $\mathbf{1 6}$ in solution, we were unable to significantly alter the rate of chiral degradation despite screening a wide variety of topically-acceptable solvents under various conditions (temperature, concentration, pH) [16]. Therefore, we switched to a suspension formulation by micronizing 16 and suspending the micronized solid in different media as a $1 \% \mathrm{w} / \mathrm{v}$ suspension (entries 2 and 3). While there was little improvement in chiral and achiral degradation in water compared to $96 \% \mathrm{EtOH}$ (aq) (entry $2 \mathrm{v}$ entry 1 ), only minor degradation was observed in oil at $23^{\circ} \mathrm{C}$ after 3 months (entry 3 ) (Table 1 ).

With these results in hand we switched our focus back on chemical modifications to prevent chiral degradation altogether. The synthetic routes for the novel six-membered warheads 37-39 were very similar to those employed for the preparation of our aspartaldehyde warhead $\mathbf{6}$ (Table 1) [17]. However, we were unable to transpose the same methodology for the preparation of the quaternary warhead $\mathbf{3 5}$ and had to change our strategy. Briefly, Fmoc protection of commercially-available (S)-2-amino-4-(tertbutoxy)-2-methyl-4-oxobutanoic acid (31) followed by reduction of the carboxylic acid $\mathbf{3 2}$ to the alcohol through reduction of the mixed anhydride using $\mathrm{NaBH}_{4}$. Fmoc deprotection afforded amine 33 followed by amide coupling with $\mathbf{3 4}$ using HATU afforded 35. Finally, oxidation of the alcohol using Dess-Martin periodinane (DMP) to the aldehyde followed by deprotection of the tert-butyl protecting group using TFA afforded $\mathbf{3 6}$ albeit in poor yield (Scheme 6).

Evaluation of the P1 warheads is shown in Table 2. The nonepimerisble quaternary warhead $\mathbf{3 6}$ (entry 3 ) was inactive despite encouraging docking studies. It is interesting to note that there is 18 fold loss of activity by switching the configuration from $R(37)$ to $S$ (38) for the 6-membered non-epimerisable warhead match pairs (entries 4 and 5). While the $R$ warhead 37 was moderately potent at $130 \mathrm{nM}$, it was unfortunately much less stable than 16 with only $64 \%$ of the compound remaining after $30 \mathrm{~d}$ at $40^{\circ} \mathrm{C}$. As the activity of our both 5-membered warhead enantiomers ( $\mathbf{1 6}$ and $\mathbf{3 6}$ ) was identical, was can safely assume that epimerization must be happening readily during our assaying of $\mathbf{1 6}$ in the enzymatic screen (entries $1 \mathrm{v}$ 2 ). Finally, the direct 6-membered cyclic analogue of our lead warhead (39) was only moderately active at $560 \mathrm{nM}$.

\section{Conclusion}

In conclusion, we have described the synthesis and stability evaluation of a novel class of potent peptidomimetic Caspase-1 inhibitors based around cyclic imidic central cores with the hemiacetal drug-form of the warhead. Our SAR and SPR exploration

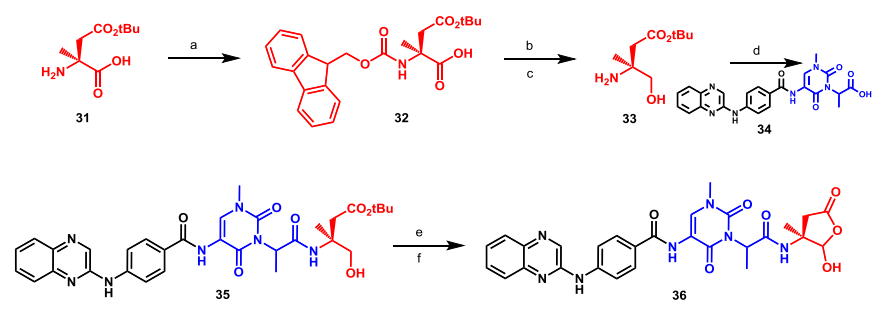

Scheme 6. Route employed for the preparation 36. Reagents and conditions: (a) FmocOSuc, $10 \% \mathrm{Na}_{2} \mathrm{CO}_{3}$ (aq), dioxane, r.t., $12 \mathrm{~h}, 85 \%$; (b) IBCF, NMM, DME, $0{ }^{\circ} \mathrm{C}, 1 \mathrm{~h}$ then add $\mathrm{NaBH}_{4}$, rt, 18 h, 98\%; (c) Pyrrolidine-MeCN (10\% v/v), r.t., 16 h, quant.; (d) HATU, NMM, DMF, $80^{\circ} \mathrm{C}, 4 \mathrm{~h}, 45 \%$; (e) Dess-Martin periodinane, DCM, $0^{\circ} \mathrm{C}$ to r.t., $2 \mathrm{~h}$, quant.; (f) 2 . TFA, DCM, rt, $1 \mathrm{~h}, 35 \%$.

Table 2

Comparison of the hemiacetal P1 Warheads.

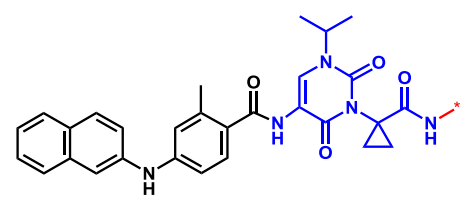

\begin{tabular}{|c|c|c|c|c|}
\hline Entry & Compound & Warhead & $\mathrm{IC}_{50}(\mathrm{nM})$ & Stability ${ }^{\mathrm{b}}$ \\
\hline 1 & 16 & & 10 & 84 \\
\hline 2 & 28 & & 10 & 88 \\
\hline $3^{a}$ & 36 & & 5900 & 79 \\
\hline 4 & 37 & & 130 & 64 \\
\hline 5 & 38 & & 2400 & 68 \\
\hline $6^{\mathrm{a}}$ & 39 & & 560 & 85 \\
\hline
\end{tabular}

\footnotetext{
${ }^{a}$ Not a direct match pair.

b Compounds were tested as $1 \% \mathrm{w} / \mathrm{w}$ solutions in $96 \% \mathrm{w} / \mathrm{w}$ EtOH (aq) at $40{ }^{\circ} \mathrm{C}$ for
} $30 \mathrm{~d}$.

Table 1

Chiral and achiral integrity study of $\mathbf{1 6}$ in different excipients.

\begin{tabular}{|c|c|c|c|c|}
\hline Entry & Excipient & Conditions & Chiral stability $(3 S, 2 R S / 3 R, 2 R S)$ & Achiral stability \\
\hline \multirow[t]{3}{*}{1} & $0.01 \% \mathrm{w} / \mathrm{w}$ in $96 \% \mathrm{EtOH}(\mathrm{aq})^{\mathrm{a}}$ & $0^{\circ} \mathrm{C}$ & $89 / 11$ after $30 \mathrm{~d}$ & $\mathrm{~N} / \mathrm{A}$ \\
\hline & & $23^{\circ} \mathrm{C}$ & $83 / 17$ after $1 \mathrm{~d}$ & $>95 \% / 6$ mo. \\
\hline & & $40^{\circ} \mathrm{C}$ & $55 / 45$ after $1 \mathrm{~d}$ & $84 \% / 30 \mathrm{~d}$ \\
\hline \multirow[t]{3}{*}{2} & $1 \% \mathrm{w} / \mathrm{w}^{\mathrm{b}}$ in water & $0^{\circ} \mathrm{C}$ & $>99 / 1$ after $30 \mathrm{~d}$ & $\mathrm{~N} / \mathrm{A}$ \\
\hline & & $23^{\circ} \mathrm{C}$ & $88 / 12$ after $1 \mathrm{~d}$ & $83 \% / 1 \mathrm{mo.}$ \\
\hline & & $40^{\circ} \mathrm{C}$ & $55 / 45$ after $1 \mathrm{~d}$ & $\mathrm{~N} / \mathrm{A}$ \\
\hline \multirow[t]{3}{*}{3} & $1 \% \mathrm{w} / \mathrm{w}^{\mathrm{b}}$ in oil Primol 352 & $0^{\circ} \mathrm{C}$ & $\mathrm{N} / \mathrm{A}$ & $\mathrm{N} / \mathrm{A}$ \\
\hline & & $23^{\circ} \mathrm{C}$ & $95 / 5$ after 3 mo. & $>95 \% / 6$ mo. \\
\hline & & $40^{\circ} \mathrm{C}$ & $92 / 8$ after 1 mo. & $\mathrm{N} / \mathrm{A}$ \\
\hline
\end{tabular}

a Similar results were obtained in other common topical excipient medium including $\mathrm{H}_{2} \mathrm{O}$, 2-propanol, propylene glycol, schercemol.

b $\mathbf{1 6}$ was micronized and suspended in the medium; N/A = not assayed. 
drove us to identify a uracil-based P2/P3 peptidiomimetic scaffold showing excellent potency and much improved achiral stability in solution in our standard excipients. The chiral and achiral stability of the hemiacetal warhead series proved to be the most challenging aspect of our exploration. This was studied in solution and was, to some extent, controlled through micronisation of the API and suspension in an oil medium.

\section{Experimental}

${ }^{1} \mathrm{H}$ NMR spectra were recorded on a Bruker Biospin Avance 400 spectrometer unless otherwise stated. Chemical shifts are reported as $\delta$ values downfield from internal TMS in appropriate organic solutions. The abbreviations used for explaining the multiplicities were as follows: $\mathrm{s}$ =singlet, $\mathrm{d}=$ doublet, $\mathrm{t}=$ triplet, $\mathrm{q}=$ quartet, $\mathrm{m}=$ multiplet, $\mathrm{br}=$ broad. For compounds isolated as mixtures of isomers, the ${ }^{1} \mathrm{H}$ integration is described as fractional integration according to the ratio of isomers. High resolution mass (ESI HRMS) was recorded on a Thermofisher Q Exactive ${ }^{\mathrm{TM}}$ Hybrid QuadrupoleOrbitrap ${ }^{\mathrm{TM}}$ Mass Spectrometer. The relative purity and the mass of the products were confirmed by LC/MS (220 nm-420 nm) on a Waters acquity uplc photodiode array detector system using the following conditions: Column, BEH C18 50*2.1 mm; $1.8 \mu \mathrm{m}$; Solvent A, water $0.1 \%$ formic acid or water ammonium carbonate $2 \mathrm{~g} / \mathrm{L}$; Solvent $\mathrm{B}, \mathrm{CH}_{3} \mathrm{CN}$; flow rate, $0.8 \mathrm{~mL} / \mathrm{min}$; run time, $2.2 \mathrm{~min}$; gradient, from 5 to $95 \%$ solvent B; mass detector, Waters SQ detector. All compounds were purified by LC/MS on a waters Autopurification system using the following conditions: Column, Xbridge C18 150*30 mm, $5 \mu \mathrm{m}$; Solvent A, water $0.1 \%$ formic acid or water ammonium carbonate $2 \mathrm{~g} / \mathrm{L}$; Solvent $\mathrm{B}, \mathrm{CH}_{3} \mathrm{CN}$; flow rate, $50 \mathrm{~mL} / \mathrm{min}$; run time, 10 or $15 \mathrm{~min}$; with adapted isocratic elution mode; mass detector, Waters ZQ detector.

4.1. $\mathrm{N}-((3 R)-1-((2 S)-1-(((3 S)-2-h y d r o x y-5-o x o t e t r a h y d r o f u r a n-3-$ yl)amino)-1-oxopropan-2-yl)-2,5-dioxopyrrolidin-3-yl)-2naphthamide (1) was prepared in 7 steps

\subsection{1. tert-Butyl (S)-2-((R)-3-((tert-butoxycarbonyl)amino)-2,5-} dioxopyrrolidin-1-yl)propanoate (4)

To a suspension of Boc-D-Asp-OH (3, $2.0 \mathrm{~g}, 8.58 \mathrm{mmol})$ in DCM $(60 \mathrm{~mL})$ at $0{ }^{\circ} \mathrm{C}$ was added EDCI $(1.98 \mathrm{~g}, 10.2 \mathrm{mmol})$. The reaction mixture was stirred at room temperature for $1 \mathrm{~h}$, cooled at $0{ }^{\circ} \mathrm{C}$ then L-Ala-OtBu.HCl (1.48 g, $8.15 \mathrm{mmol})$ and DIPEA ( $3.0 \mathrm{~mL}, 17.1 \mathrm{mmol})$ were added successively. The reaction mixture was stirred at room temperature for $1 \mathrm{~h} 30 \mathrm{~min}$, cooled to $0^{\circ} \mathrm{C}$ then EDCI $(1.98 \mathrm{~g}$, $10.2 \mathrm{mmol}$ ) and HOBt ( $1.28 \mathrm{~g}, 9.43 \mathrm{mmol}$ ) were added. The reaction mixture was stirred at room temperature for $16 \mathrm{~h} 1 \mathrm{~N} \mathrm{HCl}$ (aq) was added and the mixture extracted using DCM. The organics were washed sequentially with a saturated solution of $\mathrm{NaHCO}_{3}(\mathrm{aq}), 1 \mathrm{~N}$ $\mathrm{HCl}$ (aq), brine, dried over $\mathrm{Na}_{2} \mathrm{SO}_{4}$, filtered and concentrated under vacuum. The residue was purified by silica gel flash chromatography (cyclohexane/EtOAc, gradient of $1 / 0$ to $1 / 1$ ) to give expected product $(1.72 \mathrm{~g}, 58 \%)$ as a white solid: $\mathrm{LCMS} \mathrm{ESI}^{+} \mathrm{m} / \mathrm{z} 343(\mathrm{M}+\mathrm{H})^{+}$, $360\left(\mathrm{M}+\mathrm{NH}_{4}\right)^{+} ;{ }^{1} \mathrm{H}$ NMR $\left.(500 \mathrm{MHz} \text {, DMSO-d })_{6}\right) 7.55(\mathrm{~d}, J=8.0 \mathrm{~Hz}$, $1 \mathrm{H}), 4.62(\mathrm{q}, J=7.2 \mathrm{~Hz}, 1 \mathrm{H}), 4.38-4.30(\mathrm{~m}, 1 \mathrm{H}), 2.97$ (dd, $J=17.5$, $9.5 \mathrm{~Hz}, 1 \mathrm{H}), 2.60(\mathrm{dd}, J=17.5,6.0 \mathrm{~Hz}, 1 \mathrm{H}), 1.38(\mathrm{~s}, 9 \mathrm{H}), 1.37(\mathrm{~s}, 9 \mathrm{H})$, $1.30(\mathrm{~d}, J=7.0 \mathrm{~Hz}, 3 \mathrm{H})$.

\subsection{2. tert-Butyl (S)-2-((R)-3-amino-2,5-dioxopyrrolidin-1-yl)} propanoate hydrochloride

To a solution of tert-butyl $(S)-2-((R)-3-(($ tert-butoxy-carbonyl) amino)-2,5-dioxopyrrolidin-1-yl)propanoate $(1.0 \mathrm{~g}, 2.92 \mathrm{mmol})$ in EtOAc $(20 \mathrm{~mL})$ at $0{ }^{\circ} \mathrm{C}$ was added $\mathrm{HCl}(4 \mathrm{~N}$ in dioxane, $7.3 \mathrm{~mL}$, $29.2 \mathrm{mmol}, 10$ eq.). The solution was stirred at room temperature for $5 \mathrm{~h}$ then at $4{ }^{\circ} \mathrm{C}$ during $15 \mathrm{~h}$. The solvent and excess $\mathrm{HCl}$ were removed under reduced pressure at room temperature. The residue was taken-up in EtOAc and the solvent evaporated again to give expected product $(850 \mathrm{mg}$ ) as beige solid. The product was engaged directly in the following step without further purification: LCMS $\mathrm{ESI}^{+} \mathrm{m} / z 243(\mathrm{M}+\mathrm{H})^{+}$.

\subsection{3. tert-Butyl (S)-2-((R)-3-(2-naphthamido)-2,5-dioxo- pyrrolidin-1-yl)propanoate}

To a solution of tert-butyl (S)-2-((R)-3-amino-2,5-dioxopyrrolidin1-yl)propanoate hydrochloride ( $810 \mathrm{mg}, 2.91 \mathrm{mmol})$ in THF $(20 \mathrm{~mL})$ at $0{ }^{\circ} \mathrm{C}$ was added 2-naphthoyl chloride $(665 \mathrm{mg}, 3.48 \mathrm{mmol})$ followed with DIPEA $(1.78 \mathrm{~mL}, 10.2 \mathrm{mmol})$. The reaction mixture was stirred at room temperature for $4 \mathrm{~h}$ then hydrolysed with $\mathrm{H}_{2} \mathrm{O}$ and extracted with EtOAc. The organic phase was washed with aq. $1 \mathrm{~N} \mathrm{HCl}$ and brine, dried over $\mathrm{Na}_{2} \mathrm{SO}_{4}$, filtered and concentrated under vacuum. The residue was purified by silica gel flash chromatography (cyclohexane/ EtOAc, gradient of $1 / 0$ to $3 / 7$ ) to give expected product ( $800 \mathrm{mg}, 59 \%$ ) as a beige solid: LCMS $\left(t_{R}=3.53 \mathrm{~min}\right.$, purity $\left.=96 \%\right) \mathrm{ESI}^{+} \mathrm{m} / z 397$ $(\mathrm{M}+\mathrm{H})^{+}, 414\left(\mathrm{M}+\mathrm{NH}_{4}\right)^{+} ;{ }^{1} \mathrm{H}$ NMR $(500 \mathrm{MHz}$, DMSO-d 6 ) $\delta 9.38(\mathrm{~d}$, $J=7.5 \mathrm{~Hz}, 1 \mathrm{H}), 8.47(\mathrm{~s}, 1 \mathrm{H}), 8.06-7.96(\mathrm{~m}, 3 \mathrm{H}), 7.92(\mathrm{dd}, J=8.5,2.0 \mathrm{~Hz}$, 1H), 7.68-7.58 (m, 2H), $4.76(\mathrm{~m}, 1 \mathrm{H}), 4.69(\mathrm{q}, J=7.0 \mathrm{~Hz}, 1 \mathrm{H}), 3.12(\mathrm{dd}$, $J=17.5,9.2, \mathrm{~Hz}, 1 \mathrm{H}), 2.81(\mathrm{dd}, J=17.5,5.2 \mathrm{~Hz}, 1 \mathrm{H}), 1.41(\mathrm{~s}, 9 \mathrm{H}), 1.39(\mathrm{t}$, $J=7.0 \mathrm{~Hz}, 3 \mathrm{H}$ ); Chiral HPLC (Chiralpak AD, $n$-heptane/2-propanol (80/ $20), 1 \mathrm{~mL} / \mathrm{min} ., t_{\mathrm{R}}=19.46 \mathrm{~min}$, purity $=100 \%$ ).

\subsection{4. (S)-2-((R)-3-(2-Naphthamido)-2,5-dioxopyrrolidin-1-yl) propanoic acid $(\mathbf{5})$}

To a solution of tert-butyl $(S)-2-((R)-3-(2-n a p h t h a m i d o)-2,5-$ dioxopyrrolidin-1-yl)propanoate $(800 \mathrm{mg}, 2.02 \mathrm{mmol})$ in DCM $(5 \mathrm{~mL})$ at $0^{\circ} \mathrm{C}$ was added TFA $(1.55 \mathrm{~mL}, 20.2 \mathrm{mmol})$. The solution was stirred at room temperature for $6 \mathrm{~h}$. Then the solvent and excess TFA were concentrated under vacuum. The residue was coevaporated with DCM, then with EtOAc, and dried under vacuum. Expected product was isolated as a beige solid (690 mg, quant.) and was used without further purification.

\subsection{5. tert-Butyl (S)-3-(((allyloxy)carbonyl)amino)-4,4-diethoxy-}

butanoate (6) was prepared in 4 steps

Step 1: To a cooled $\left(4^{\circ} \mathrm{C}\right)$ solution of L-aspartic acid 4-tert-butyl ester $(25.0 \mathrm{~g}, 132.1 \mathrm{mmol})$ in $\mathrm{THF} / \mathrm{H}_{2} \mathrm{O}(80 \mathrm{~mL} / 240 \mathrm{~mL})$ were added sodium bicarbonate $(44.4 \mathrm{~g}, 528.5 \mathrm{mmol})$ and allyl chloroformate $(25.3 \mathrm{~mL}, 237.8 \mathrm{mmol})$. After $2 \mathrm{~h} 30$ of stirring at room temperature, the medium was extracted with EtOAc (three times). The aqueous layer was acidified with aqueous $\mathrm{HCl}(6 \mathrm{~N})$ until obtaining $\mathrm{pH} 2$. The aqueous layer was extracted (EtOAc, three times). The combined organic layer was dried over sodium sulphate, filtered and concentrated in vacuo to afford (S)-2-(((allyloxy)-carbonyl)amino)4-(tert-butoxy)-4-oxobutanoic acid $(29.2 \mathrm{~g}, 80 \%)$ as a pale yellow oil: LCMS ESI ${ }^{-} \mathrm{m} / \mathrm{z} 272.4(\mathrm{M}-\mathrm{H})^{-} ;{ }^{1} \mathrm{H}$ NMR $\left(\mathrm{CDCl}_{3}, 500 \mathrm{MHz}\right)$ $\delta 5.87-5.96(\mathrm{~m}, 1 \mathrm{H}), 5.74(\mathrm{bd}, J=8.5 \mathrm{~Hz}, 1 \mathrm{H}), 5.31(\mathrm{~m}, 1 \mathrm{H}), 5.22(\mathrm{~m}$, $1 \mathrm{H}), 4.55-4.65(\mathrm{~m}, 3 \mathrm{H}), 2.97(\mathrm{dd}, J=17.0 \mathrm{~Hz}$ and $4.0 \mathrm{~Hz}, 1 \mathrm{H}), 2.76$ (dd, $J=17.0 \mathrm{~Hz}$ and $5.0 \mathrm{~Hz}, 1 \mathrm{H}), 1.44(\mathrm{~s}, 9 \mathrm{H})$.

Step 2: To a cooled $\left(4^{\circ} \mathrm{C}\right)$ solution of $(S)-2-((($ allyloxy $)$ carbonyl $)$ amino)-4-(tert-butoxy)-4-oxobutanoic acid (29.1 g, $106.4 \mathrm{mmol})$ in DCM $(600 \mathrm{~mL})$ were added N,O-dimethylhydroxylamine hydrochloride $(12.4 \mathrm{~g}, 127.8 \mathrm{mmol}), 4$-methylmorpholine $(14.05 \mathrm{~mL}$, $127.8 \mathrm{mmol}$ ) and 1,(3-dimethylaminopropyl)-3-ethylcarbodiimide hydrochloride ( $24.5 \mathrm{~g}, 127.8 \mathrm{mmol})$. After $3 \mathrm{~h}$ of stirring at room temperature, the medium was washed with aqueous $\mathrm{HCl}(0.2 \mathrm{~N})$, with water, with brine, dried over sodium sulphate, filtered and concentrated in vacuo to afford tert-butyl (S)-3-(((allyloxy) carbonyl)amino)-4-(methoxy(methyl)amino)-4-oxo-butanoate $(29.2 \mathrm{~g}, 86 \%)$ as a yellow oil: $\mathrm{LCMS} \mathrm{ESI}^{+} \mathrm{m} / \mathrm{z} 317.3(\mathrm{M}+\mathrm{H})^{+} ;{ }^{1} \mathrm{H}$ NMR $\left(\mathrm{CDCl}_{3}, 500 \mathrm{MHz}\right) \delta 5.85-5.93(\mathrm{~m}, 1 \mathrm{H}), 5.59(\mathrm{br} \mathrm{d}, J=8.5 \mathrm{~Hz}, 1 \mathrm{H})$, $5.30(\mathrm{~m}, 1 \mathrm{H}), 5.19(\mathrm{~m}, 1 \mathrm{H}), 5.00(\mathrm{~m}, 1 \mathrm{H}), 4.56(\mathrm{~m}, 2 \mathrm{H}), 3.78(\mathrm{~s}, 3 \mathrm{H})$, 
$3.21(\mathrm{~s}, 3 \mathrm{H}), 2.70(\mathrm{dd}, J=15.0 \mathrm{~Hz}$ and $5.5 \mathrm{~Hz}, 1 \mathrm{H}), 2.54$ (dd, $J=15.0 \mathrm{~Hz}$ and $6.5 \mathrm{~Hz}, 1 \mathrm{H}), 1.43(\mathrm{~s}, 9 \mathrm{H})$.

Step 3: A solution of lithium aluminium hydride ( $2 \mathrm{~N}$ in THF, $5.93 \mathrm{~mL}, 11.8 \mathrm{mmol})$ was added dropwise to a cooled $\left(-78^{\circ} \mathrm{C}\right)$ solution of tert-butyl (S)-3-(((allyloxy)carbonyl)amino)-4-(methox$\mathrm{y}$ (methyl)amino)-4-oxobutanoate $(5.0 \mathrm{~g}, 15.8 \mathrm{mmol})$ in anhydrous THF $(65 \mathrm{~mL})$. The mixture was stirred at $-78^{\circ} \mathrm{C}$ for $2 \mathrm{~h}$, then aqueous $\mathrm{HCl}(1 \mathrm{~N})$ was slowly added to the medium and the temperature was allowed to warm to $0^{\circ} \mathrm{C}$. The mixture was diluted with EtOAc. The aqueous layer was extracted (EtOAc, two times). The combined organic layer was washed with aqueous $\mathrm{HCl}(0.5 \mathrm{~N})$, with brine, dried over sodium sulphate, filtered and concentrated in vacuo to afford tert-butyl (S)-3-(((allyloxy)carbonyl)amino)-4oxobutanoate $(3.4 \mathrm{~g}, 83 \%)$ as a pale pink oil: ${ }^{1} \mathrm{H}$ NMR $\left(\mathrm{CDCl}_{3}\right)$ $\delta 9.66(\mathrm{~s}, 1 \mathrm{H}), 5.83(\mathrm{br} \mathrm{d}, 1 \mathrm{H}), 5.99-5.82(\mathrm{~m}, 1 \mathrm{H}), 5.33(\mathrm{~m}, 1 \mathrm{H}), 5.24$ $(\mathrm{m}, 1 \mathrm{H}), 4.61(\mathrm{~m}, 2 \mathrm{H}), 4.36(\mathrm{~m}, 1 \mathrm{H}), 2.96(\mathrm{dd}, J=17.2 \mathrm{~Hz}$ and $4.5 \mathrm{~Hz}$, $1 \mathrm{H}), 2.76(\mathrm{dd}, J=17.2 \mathrm{~Hz}$ and $5.1 \mathrm{~Hz}, 1 \mathrm{H}), 1.43(\mathrm{~s}, 9 \mathrm{H})$.

Step 4: To a freshly prepared solution of tert-butyl (S)-3-(((allyloxy)carbonyl)amino)-4-oxobutanoate $(3.40 \mathrm{~g}, 13.23 \mathrm{mmol})$ in absolute ethanol $(11 \mathrm{~mL})$ were added, under Argon atmosphere, triethyl orthoformate $(5.62 \mathrm{~mL}, 33.09 \mathrm{mmol}), p$-toluenesulfonic acid ( $45 \mathrm{mg}, 0.26 \mathrm{mmol}$ ) and $3 \AA$ molecular sieves. After stirring at room temperature overnight, the mixture was filtered over a pad of Celite and rinsed with $\mathrm{EtOH}$. The filtrate was concentrated under reduced pressure and co-evaporated with toluene (three times). Purification of the residue by flash chromatography on silica gel (cyclohexane-EtOAc 9/1 to 8/2) afforded the title compound (6, $4.05 \mathrm{~g}, 92 \%)$ as a colorless oil $):{ }^{1} \mathrm{H}$ NMR $\left(\mathrm{CDCl}_{3}\right) \delta 5.83-6.00(\mathrm{~m}, 1 \mathrm{H})$, $5.30(\mathrm{~d}, J=17.4 \mathrm{~Hz}, 1 \mathrm{H}), 5.23(\mathrm{br} \mathrm{d}, J=8.5 \mathrm{~Hz}, 1 \mathrm{H}), 5.21(\mathrm{~m}, 1 \mathrm{H}), 4.56$ $(\mathrm{m}, 2 \mathrm{H}), 4.49(\mathrm{~m}, 1 \mathrm{H}), 4.22-4.11(\mathrm{~m}, 1 \mathrm{H}), 3.78-3.65(\mathrm{~m}, 2 \mathrm{H})$, 3.61-3.48 (m, 2H), 2.56 (dd, $J=15.6 \mathrm{~Hz}$ and $5.7 \mathrm{~Hz}, 1 \mathrm{H}), 2.45$ (dd, $J=15.6 \mathrm{~Hz}$ and $7.2 \mathrm{~Hz}, 1 \mathrm{H}), 1.44(\mathrm{~s}, 9 \mathrm{H}), 1.21(\mathrm{~m}, 6 \mathrm{H}) ;{ }^{13} \mathrm{C} \mathrm{NMR}$ $\left(101 \mathrm{MHz}\right.$, DMSO- $\left.d_{6}\right) \delta 170.12,155.44,133.78,116.66,102.57,79.66$, 64.16, 63.39, 62.22, 50.44, 36.09, 27.69, 15.15; $[\alpha]_{\mathrm{D}}^{20}=-18.3^{\circ}$ (c = $16 \mathrm{~g} / \mathrm{L}, \mathrm{MeCN})$; HRMS: $(\mathrm{M}+\mathrm{H})^{+}$calculated for $\mathrm{C}_{16} \mathrm{H}_{29} \mathrm{NO}_{6}$ 331.1995; found $354.1886(\mathrm{M}+\mathrm{Na})^{+}, 685.3883(2 \mathrm{M}+\mathrm{Na})^{+}, 230.1022$ cyclized warhead eg., $(\mathrm{M}(-\mathrm{OEt},-\mathrm{BBu})+\mathrm{H})^{+}$.

4.1.6. $N-((3 R)-1-((2 S)-1-(((3 S)-2-E t h o x y-5-o x o t e t r a h y d r o-f u r a n-3-$ yl)amino)-1-oxopropan-2-yl)-2,5-dioxopyrrolidin-3-yl)-2naphthamide (7)

To a degassed solution (argon sparging, $5 \mathrm{~min}$ ) of $\mathrm{N}$-Alloc protected warhead $\mathbf{6}(803 \mathrm{mg}, 2.42 \mathrm{mmol}$ ) and dimethylbarbituric acid (315 $\mathrm{mg}, 2.02 \mathrm{mmol})$ in DCM $(15 \mathrm{~mL})$ at room temperature was added $\mathrm{Pd}\left(\mathrm{PPh}_{3}\right)_{4}(42 \mathrm{mg}, 36 \mu \mathrm{mol}, 0.015 \mathrm{eq})$. The solution was stirred at room temperature for $15 \mathrm{~min}$ (TLC (cyclohexane/EtOAc, 7/ 3 ) showed deprotection was complete). At the same time, the acid derivative obtained above $(687 \mathrm{mg}, 2.02 \mathrm{mmol}$ ) in solution in a mixture DCM/DMF $(8 \mathrm{~mL} / 7 \mathrm{~mL})$ at $0{ }^{\circ} \mathrm{C}$ was treated with EDCI (580 mg, $3.03 \mathrm{mmol}$ ) and HOBt (273 $\mathrm{mg}, 2.02 \mathrm{mmol}$ ). The reaction mixture was stirred for $5 \mathrm{~min}$. Then, the solution of the amine prepared above was added dropwise. The reaction mixture was stirred at room temperature for $16 \mathrm{~h}$. After removal of solvents, the residue was taken-up in EtOAc and aq. saturated $\mathrm{NH}_{4} \mathrm{Cl}$. After separation, aqueous phase was extracted with EtOAc. The combined organics were washed sequentially with aq. sat. $\mathrm{NaHCO}_{3}, 1 \mathrm{~N}$ $\mathrm{HCl}$ and brine, then dried over $\mathrm{Na}_{2} \mathrm{SO}_{4}$, filtered and concentrated under vacuum. The residue was purified by silica gel flash chromatography (cyclohexane/EtOAc, gradient of $1 / 0$ to $1 / 4$ ). The expected product was isolated as a beige solid ( $830 \mathrm{mg}, 72 \%): R_{\mathrm{f}}=0.3$ (cyclohexane/EtOAc 2/3); LCMS $\left(t_{\mathrm{R}}=3.67 \mathrm{~min}\right.$, purity $\left.=100 \%\right) \mathrm{ESI}^{+}$ $\mathrm{m} / \mathrm{z} 570(\mathrm{M}+\mathrm{H})^{+} ;{ }^{1} \mathrm{H}$ NMR $\left(500 \mathrm{MHz}, \mathrm{CDCl}_{3}\right) \delta 8.31(\mathrm{~s}, 1 \mathrm{H})$, $7.84-7.72(\mathrm{~m}, 5 \mathrm{H}), 7.54-7.46(\mathrm{~m}, 2 \mathrm{H}), 7.16(\mathrm{~d}, J=8.5 \mathrm{~Hz}, 1 \mathrm{H}), 4.92(\mathrm{q}$, $J=7.3 \mathrm{~Hz}, 1 \mathrm{H}), 4.68(\mathrm{~m}, 1 \mathrm{H}), 4.60-4.52(\mathrm{~m}, 2 \mathrm{H}), 3.83-3.70(\mathrm{~m}, 2 \mathrm{H})$, $3.65-3.55(\mathrm{~m}, 2 \mathrm{H}), 3.24$ (dd, $J=18.5,9.5 \mathrm{~Hz}, 1 \mathrm{H}), 2.99$ (dd, $J=18.5$,
$5.5 \mathrm{~Hz}, 1 \mathrm{H}), 2.70-2.55(\mathrm{~m}, 2 \mathrm{H}), 1.71(\mathrm{~d}, J=7.0 \mathrm{~Hz}, 3 \mathrm{H}), 1.39(\mathrm{~s}, 9 \mathrm{H})$, $1.24(\mathrm{t}, J=7.0 \mathrm{~Hz}, 3 \mathrm{H}), 1.23(\mathrm{t}, J=7.0 \mathrm{~Hz}, 3 \mathrm{H})$.

4.1.7. $N-((3 R)-1-((2 S)-1-(((3 S)-2-E t h o x y-5-o x o t e t r a h y d r o-f u r a n-3-$ yl)amino)-1-oxopropan-2-yl)-2,5-dioxopyrrolidin-3-yl)-2naphthamide (8)

To a solution of $N-((3 R)-1-((2 S)-1-(((3 S)-2-e t h o x y-5-$ oxotetrahydrofuran-3-yl)amino)-1-oxopropan-2-yl)-2,5-dioxopyrrolidin-3-yl)-2-naphthamide $(830 \mathrm{mg}, 1.46 \mathrm{mmol})$ in DCM $(15 \mathrm{~mL})$ at $0^{\circ} \mathrm{C}$ was added TFA $(1.12 \mathrm{~mL}, 14.6 \mathrm{mmol}, 10 \mathrm{eq})$. The mixture was stirred for $45 \mathrm{~min}$ at $0^{\circ} \mathrm{C}$, then the solvent and excess TFA were removed. The residue was co-evaporated with DCM, then purified by flash chromatography ( $40 \mathrm{~g}$ silica column, cyclohexane/ AcOEt, gradient of $1 / 0$ to $0 / 1$ ) and then on preparative TLC (cyclohexane/AcOEt $1 / 4$ ) to give the desired product as a white solid (405 mg, 59\%): LCMS $\left(t_{\mathrm{R}}=3.18 \mathrm{~min}\right.$, purity $\left.=93 \%\right) \mathrm{ESI}^{+} \mathrm{m} / \mathrm{z} 468.1$ $(\mathrm{M}+\mathrm{H})^{+} ;{ }^{1} \mathrm{H} \mathrm{NMR}\left(\mathrm{CDCl}_{3}, 500 \mathrm{MHz}\right) \delta 8.38(\mathrm{~s}, 1 \mathrm{H}), 7.90(\mathrm{~d}, J=8.0 \mathrm{~Hz}$, 1H) $7.85(\mathrm{~d}, J=7.0 \mathrm{~Hz}, 1 \mathrm{H}), 7.80-7.65(\mathrm{~m}, 4 \mathrm{H}), 7.60-7.40(\mathrm{~m}, 2 \mathrm{H})$, $5.50(\mathrm{~s}, 1 \mathrm{H}), 4.95(\mathrm{q}, J=7.3 \mathrm{~Hz}, 1 \mathrm{H}), 4.44(\mathrm{t}, J=6.7 \mathrm{~Hz}, 1 \mathrm{H}), 4.29(\mathrm{~m}$, $1 \mathrm{H}), 3.85(\mathrm{~m}, 1 \mathrm{H}), 3.66(\mathrm{~m}, 1 \mathrm{H}), 3.18(\mathrm{dd}, J=18.5,9.5 \mathrm{~Hz}, 1 \mathrm{H}), 3.02$ (dd, $J=18.5,5.0 \mathrm{~Hz}, 1 \mathrm{H}$ ), 2.91 (dd, $J=18.0,8.0 \mathrm{~Hz}, 1 \mathrm{H}$ ), 2.60 (dd, $J=18.0,2.0 \mathrm{~Hz}, 1 \mathrm{H}), 1.63(\mathrm{~d}, J=7.0 \mathrm{~Hz}, 3 \mathrm{H}), 1.22(\mathrm{t}, J=7.2 \mathrm{~Hz}, 3 \mathrm{H})$.

4.1.8. $N-((3 R)-1-((2 S)-1-(((3 S)-2-H y d r o x y-5-o x o t e t r a h y d r o-f u r a n-$ 3-yl)amino)-1-oxopropan-2-yl)-2,5-dioxopyrrolidin-3-yl)-2naphthamide (1)

To a solution of $N-((3 \mathrm{R})-1-((2 S)-1-(((3 S)-2-e t h o x y-5-$ oxotetrahydrofuran-3-yl)amino)-1-oxopropan-2-yl)-2,5-dioxopyrrolidin-3-yl)-2-naphthamide (330 $\mathrm{mg}, 0.706 \mathrm{mmol}$ ) in $\mathrm{MeCN}$ ( $3.5 \mathrm{~mL}$ ) at $0{ }^{\circ} \mathrm{C}$ was added aq. $2 \mathrm{~N} \mathrm{HCl}(3.5 \mathrm{~mL}, 7.1 \mathrm{mmol}, 10 \mathrm{eq})$. The reaction mixture was stirred at room temperature for $4 \mathrm{~h}$, then extracted with EtOAc. The organic phase was then dried over $\mathrm{Na}_{2} \mathrm{SO}_{4}$, filtered and concentrated under vacuum. The residue was purified by preparative $\mathrm{HPLC}$ (eluent $\mathrm{MeCN} / \mathrm{H}_{2} \mathrm{O} / \mathrm{HCO}_{2} \mathrm{H}$ ). The pure fractions were frozen and lyophilised to yield expected product $(100 \mathrm{mg}, 32 \%)$ as a white solid: $\mathrm{LCMS}\left(t_{\mathrm{R}}=3.97 \mathrm{~min}\right.$, purity $\left.=100 \%\right)$ $\mathrm{ESI}^{+} \mathrm{m} / z 440(\mathrm{M}+\mathrm{H}){ }^{+} ;{ }^{1} \mathrm{H}$ NMR $(500 \mathrm{MHz}, \mathrm{MeOD}) \delta 8.45(\mathrm{~s}, 1 \mathrm{H})$, 8.03-7.90 (m, 4H), 7.63-7.55 (m, 2H), 4.83 (overlap with HDO signal, $1 \mathrm{H}), 4.65(\mathrm{dd}, J=10.0,4.0 \mathrm{~Hz}, 1 \mathrm{H}), 4.63(\mathrm{~m}, 1 \mathrm{H}), 4.36(\mathrm{~m}, 1 \mathrm{H})$, $3.18(\mathrm{dd}, J=18.0,9.5 \mathrm{~Hz}, 1 \mathrm{H}), 2.89 \& 2.86(2 \mathrm{t}, J=4.8 \mathrm{~Hz}, 1 \mathrm{H}), 2.67 \&$ $2.64(2 \mathrm{dd}, J=6.0,4.0 \mathrm{~Hz}, 1 \mathrm{H}), 2.59-2.51(\mathrm{~m}, 1 \mathrm{H}), 1.60 \& 1.61(2 \mathrm{~d}$, $J=7.5 \mathrm{~Hz}, 3 \mathrm{H}) ;{ }^{13} \mathrm{C}$ NMR (DMSO- $\left.d_{6}\right) \delta 175.43,174.80,168.71,166.81$, 134.49, 132.13, 129.01, 128.20, 128.19, 128.10, 127.73, 127.01, 123.80, 102.69, 52.93, 49.20, 48.13, 35.24, 32.79, 13.57; HRMS: $(\mathrm{M}+\mathrm{H})^{+}$ calculated for $\mathrm{C}_{22} \mathrm{H}_{21} \mathrm{~N}_{3} \mathrm{O}_{7} 440.1379$; found 440.14505 .

4.2. $N-((3 R)-1-((2 S)-1-(((3 S)-2-h y d r o x y-5-o x o t e t r a h y d r o f u r a n-3-$ yl)amino)-1-oxopropan-2-yl)-2,6-dioxopiperidin-3-yl)-2naphthamide (2) was prepared in 7 steps

\subsection{1. tert-Butyl (S)-2-((R)-3-(((benzyloxy)carbonyl)amino)-2,6- dioxopiperidin-1-yl)propanoate}

$\mathrm{K}_{2} \mathrm{CO}_{3}$ (55.3 mg, $0.400 \mathrm{mmol}$ ) was added to a solution of $(R)-3-$ $\mathrm{N}$-Cbz-amino-2,6-dioxo-piperidine $(\mathbf{9}, 100 \mathrm{mg}, 0.381 \mathrm{mmol})$ and tert-butyl (R)-2-(((trifluoromethyl)-sulfonyl)oxy)propanoate $(117 \mathrm{mg}, 0.419 \mathrm{mmol})$ in anhydrous $\mathrm{MeCN}(2.0 \mathrm{~mL})$ at $0^{\circ} \mathrm{C}$. The reaction mixture was allowed to stir at r.t. for $30 \mathrm{~h}$. The mixture was diluted with EtOAc, washed with $1 \mathrm{~N} \mathrm{HCl} \mathrm{(aq)} \mathrm{and} \mathrm{the} \mathrm{organic} \mathrm{phase}$ was dried over $\mathrm{Na}_{2} \mathrm{SO}_{4}$ and concentrated under reduce pressure. The residue was purified by preparative TLC (cyclohexane/EtOAc, 1/ 1) to give the title compound as yellow oil (79 mg, 53\%): LCMS $\left(t_{\mathrm{R}}=3.47 \mathrm{~min}\right.$, purity $=100 \%(\mathrm{ESLD}) \mathrm{m} / z\left(\mathrm{ESI}^{+}\right) 408.2\left(\mathrm{M}+\mathrm{NH}_{4}^{+}\right)^{+}$, $391.1(\mathrm{M}+\mathrm{H})^{+}, \quad 335.1\left(\mathrm{M}-\mathrm{C}_{4} \mathrm{H}_{9}\right) ;{ }^{1} \mathrm{H} \quad \mathrm{NMR}\left(\mathrm{CDCl}_{3}, 500 \mathrm{MHz}\right)$ $\delta 7.28-7.40(\mathrm{~m}, 5 \mathrm{H}), 5.70(\mathrm{br} \mathrm{s}, 1 \mathrm{H}), 5.09-5.24(\mathrm{~m}, 1 \mathrm{H}$, superposition with benzylic $\left.\mathrm{CH}_{2}\right), 5.13(\mathrm{~s}, 2 \mathrm{H}), 4.24-4.40(\mathrm{~m}, 1 \mathrm{H}), 2.62-2.93(\mathrm{~m}$, 
$2 \mathrm{H}), 2.44-2.59(\mathrm{~m}, 1 \mathrm{H}), 1.74-1.92(\mathrm{~m}, 1 \mathrm{H}), 1.43(\mathrm{~m}, 3 \mathrm{H}$, superposition avec $t-\mathrm{Bu}), 1.40(\mathrm{~s}, 9 \mathrm{H})$; Chiral HPLC (Chiralpak AD, $n-$ heptane/2-propanol $(80 / 20), 1 \mathrm{~mL} / \mathrm{min} . t_{\mathrm{R}}=9.91$ (minor) \& 14.27 (major), ratio $2 / 98$, e.e. $=96 \%$.

\subsection{2. (S)-2-((R)-3-(((Benzyloxy)carbonyl)amino)-2,6-dioxo-}

piperidin-1-yl)propanoic acid (10)

Under argon, to a solution of tert-butyl $(S)-2-((R)-3-((($ benzyloxy)carbonyl)amino)-2,6-dioxopiperidin-1-yl)propan-oate $(412 \mathrm{mg}, 1.06 \mathrm{mmol})$ in $\mathrm{DCM}(10 \mathrm{~mL})$ at $0{ }^{\circ} \mathrm{C}$ was added TFA $(2.44 \mathrm{~mL}, 31.66 \mathrm{mmol})$. The reaction mixture was stirred at room temperature for $4 \mathrm{~h}$. TLC monitoring (cyclohexane/EtOAc, 1/1) showed complete conversion. The reaction mixture was concentrated in vacuum. The residue was co-evaporated with DCM 4 times to remove residual TFA. The residue was taken-up in EtOAc and precipitated in pentane. The off-white solid was filtered, rinsed with pentane and then evaporated to dryness to give expected product (416 $\mathrm{mg}$, quant) that was used without further purification: LCMS $\left(t_{\mathrm{R}}=2.79 \mathrm{~min}\right.$, purity $\left.=100 \%\right) \mathrm{ESI}^{-} \mathrm{m} / z 333.1(\mathrm{M}-\mathrm{H})^{-} ;{ }^{1} \mathrm{H}$ NMR $\left(300 \mathrm{MHz}, \mathrm{DMSO}-d_{6}\right) \delta 12.54(\mathrm{br} \mathrm{s}, 1 \mathrm{H}), 7.70(\mathrm{~d}, J=8.7 \mathrm{~Hz}, 1 \mathrm{H})$, 7.28-7.42 (m, 5H), $5.07(\mathrm{~s}, 2 \mathrm{H}), 5.02-5.14(\mathrm{~m}, 1 \mathrm{H}), 4.35-4.55(\mathrm{~m}$, $1 \mathrm{H}), 2.82-3.00(\mathrm{~m}, 1 \mathrm{H}), 2.60-2.77(\mathrm{~m}, 1 \mathrm{H}), 1.90-2.02(\mathrm{~m}, 2 \mathrm{H}), 1.29$ $(\mathrm{d}, J=6.9 \mathrm{~Hz}, 3 \mathrm{H})$.

\subsection{3. tert-Butyl (S)-3-((S)-2-((R)-3-(((benzyloxy)carbonyl)-} amino)-2,6-dioxopiperidin-1-yl)propanamido)-4,4diethoxybutanoate (11)

To a degassed solution (argon bubbling, $5 \mathrm{~min}$ ) of Warhead 6 (419 mg, $1.27 \mathrm{mmol}, 1.2$ eq.) and dimethylbarbituric acid (132 mg, $0.845 \mathrm{mmol}, 0.8$ eq.) in DCM $(10 \mathrm{~mL})$ at room temperature was added $\mathrm{Pd}\left(\mathrm{PPh}_{3}\right)_{4}(12 \mathrm{mg}, 10.6 \mu \mathrm{mol}, 0.01$ eq.). The solution was stirred at room temperature for $10 \mathrm{~min}$ (TLC monitoring (cyclohexane/EtOAc, 7/3) showed deprotection was complete). The acid derivative (353 $\mathrm{mg}, 1.06 \mathrm{mmol}$ ) in solution in a mixture DCM/DMF $(5 \mathrm{~mL} / 1 \mathrm{~mL})$ was then added followed by HOBt ( $143 \mathrm{mg}, 1.06 \mathrm{mmol}$ ) and $\mathrm{EDCl}$ (243 $\mathrm{mg}, 1.27 \mathrm{mmol})$. The reaction mixture was stirred at room temperature for $20 \mathrm{~h}$. After removal of solvents, the residue was taken-up in EtOAc and aq. saturated $\mathrm{NH}_{4} \mathrm{Cl}$. After separation, aqueous phase was extracted with EtOAc. The combined organics were washed sequentially with aq. sat. $\mathrm{NaHCO}_{3}, 1 \mathrm{~N} \mathrm{HCl}$, and brine, then dried over $\mathrm{Na}_{2} \mathrm{SO}_{4}$, filtered and concentrated under vacuum. The residue was purified by silica gel flash chromatography (cyclohexane/EtOAc, gradient from $1 / 0$ to $1 / 4$ ). Expected product was isolated as a white solid ( $371 \mathrm{mg}, 62 \%)$ : LCMS $\left(t_{\mathrm{R}}=3.75 \mathrm{~min}\right.$, purity $=90 \%) \mathrm{ESI}^{+} \mathrm{m} / z$ 564.4 $(\mathrm{M}+\mathrm{H})^{+} ;{ }^{1} \mathrm{H}$ NMR $\left(300 \mathrm{MHz}, \mathrm{CDCl}_{3}\right)$ $\delta 7.30-7.40(\mathrm{~m}, 5 \mathrm{H}), 6.73 \& 6.58(2 \mathrm{~d}, J=8.6 \mathrm{~Hz}, 1 \mathrm{H}), 5.70(\mathrm{br} \mathrm{s}, 1 \mathrm{H})$, $5.15-5.28(\mathrm{~m}, 1 \mathrm{H}), 5.13(\mathrm{~s}, 2 \mathrm{H}), 4.25-4.50(\mathrm{~m}, 3 \mathrm{H}), 3.60-3.80(\mathrm{~m}$, $2 \mathrm{H}), 3.48-3.60(\mathrm{~m}, 2 \mathrm{H}), 2.67-2.95(\mathrm{~m}, 2 \mathrm{H}), 2.37-2.58(\mathrm{~m}, 3 \mathrm{H})$, $1.90-2.07(\mathrm{~m}, 1 \mathrm{H}), 1.50(\mathrm{~d}, J=6.9 \mathrm{~Hz}, 3 \mathrm{H}), 1.41(\mathrm{~s}, 9 \mathrm{H}), 1.13-1.23(\mathrm{~m}$, $6 \mathrm{H})$.

4.2.4. tert-Butyl (S)-3-((S)-2-((R)-3-amino-2,6-dioxopiperidin-1yl)propanamido)-4,4-diethoxybutanoate

To a degassed solution of tert-butyl $(S)-3-((S)-2-((R)-3-((($ benzyloxy)carbonyl)amino)-2,6-dioxopiperidin-1-yl)propan-amido)4,4-diethoxybutanoate ( $371 \mathrm{mg}, 0.658 \mathrm{mmol}$ ) in EtOH $(20 \mathrm{~mL}) 20 \%$ $\mathrm{Pd}(\mathrm{OH})_{2} / \mathrm{C}(100 \mathrm{mg})$ was added. The reaction mixture was hydrogenated under $1 \mathrm{~atm}$ of $\mathrm{H}_{2}$ at room temperature for $6 \mathrm{~h}$. The reaction mixture was then filled with argon. TLC monitoring of the reaction (cyclohexane/EtOAc, 3/7) showed a complete conversion. The medium was filtered on Celite and rinsed with EtOH. After evaporation to dryness, the brown solid obtained ( $290 \mathrm{mg}$, quant.) was used without further purification in the next step: LCMS $\left(t_{\mathrm{R}}=2.56 \mathrm{~min}\right.$, purity $\left.=90 \%\right), \mathrm{ESI}^{+} \mathrm{m} / z 430.3(\mathrm{M}+\mathrm{H})^{+} ;{ }^{1} \mathrm{H}$ NMR $\left(300 \mathrm{MHz}, \mathrm{CDCl}_{3}\right) \delta 6.68(\mathrm{~d}, J=8.5 \mathrm{~Hz}, 1 \mathrm{H}), 5.16-5.28(\mathrm{~m}, 1 \mathrm{H})$,
4.46-4.54 (m, 1H), 4.27-4.38 (m, $1 \mathrm{H}), 3.42-3.80(\mathrm{~m}, 5 \mathrm{H})$, 2.80-2.93 (m, 1H), 2.60-2.77 (m, 1H), 2.38-2.56 (m, 2H), $1.19(\mathrm{t}$, $J=7.0 \mathrm{~Hz}, 6 \mathrm{H}), 1.43(\mathrm{~s}, 9 \mathrm{H}), 1.50(\mathrm{br} \mathrm{d}, 3 \mathrm{H}), 1.64-1.77(\mathrm{~m}, 2 \mathrm{H})$, $1.80-1.98(\mathrm{~m}, 1 \mathrm{H}), 2.16-2.28(\mathrm{~m}, 1 \mathrm{H})$.

4.2.5. tert-Butyl (S)-3-((S)-2-((R)-3-(2-naphthamido)-2,6dioxopiperidin-1-yl)propanamido)-4,4-diethoxybutanoate (12)

To a solution of the tert-butyl $(S)-3-((S)-2-((R)-3-((($ benzyloxy) carbonyl)amino)-2,6-dioxopiperidin-1-yl)propan-amido)-4,4diethoxybutanoate obtained above $(283 \mathrm{mg}, 0.659 \mathrm{mmol})$ in THF $(5 \mathrm{~mL})$ cooled to $0^{\circ} \mathrm{C}$, were successively added DIPEA $(0.207 \mathrm{~mL}$, $1.19 \mathrm{mmol}$ ) and 2-naphthoyl chloride $(163 \mathrm{mg}, 0.856 \mathrm{mmol})$. The reaction mixture was stirred at room temperature for $4 \mathrm{~h}$. LCMS monitoring showed disappearance of starting material. The reaction mixture was then directly concentrated under reduced pressure with silica. The residue was purified by silica gel flash chromatography (cyclohexane/EtOAc, gradient of 9/1 to 3/7) to give the expected product ( $318 \mathrm{mg}, 83 \%$ ) as a pink solid: LCMS $\left(t_{\mathrm{R}}=3.15 \mathrm{~min}\right.$, purity $\left.=99 \%\right) \mathrm{ESI}^{+} \mathrm{m} / \mathrm{z} 584.2(\mathrm{M}+\mathrm{H})^{+} ;{ }^{1} \mathrm{H}$ NMR $\left(300 \mathrm{MHz}, \mathrm{DMSO}-d_{6}\right) \delta 9.01 \& 9.13(2 \mathrm{~d}, J=8.5 \mathrm{~Hz}, 1 \mathrm{H}), 8.50(\mathrm{~s}, 1 \mathrm{H})$, $7.92-8.10(\mathrm{~m}, 4 \mathrm{H}), 7.58-7.68(\mathrm{~m}, 2 \mathrm{H}), 7.48 \& 7.57(2 \mathrm{~d}, J=8.4 \mathrm{~Hz}$, $1 \mathrm{H}), 4.92-5.12(\mathrm{~m}, 1.5 \mathrm{H}), 4.80-4.92(\mathrm{~m}, 0.2 \mathrm{H}), 4.42(\mathrm{~d}, J=5.1 \mathrm{~Hz}$, $1 \mathrm{H}), 4.36-4.26(\mathrm{~m}, 0.3 \mathrm{H}), 4.25-4.12(\mathrm{~m}, 1 \mathrm{H}), 3.68-3.39(\mathrm{~m}, 4 \mathrm{H})$, 2.54-2.95 (m, 2H), 2.00-2.50 (m, 4H), 1.30-1.41 (s and $\mathrm{m}, 9 \mathrm{H}$ and $3 \mathrm{H}), 1.04-1.13(\mathrm{~m}, 6 \mathrm{H})$.

4.2.6. $N-((3 R)-1-((2 S)-1-(((3 S)-2-e t h o x y-5-o x o t e t r a h y d r o f u r a n-3-$ yl)amino)-1-oxopropan-2-yl)-2,6-dioxopiperidin-3-yl)-2naphthamide

To tert-butyl (S)-3-((S)-2-((R)-3-(2-naphthamido)-2,6-dioxopiperidin-1-yl)propanamido)-4,4-diethoxybutanoate $(12,314 \mathrm{mg}$, $0.534 \mathrm{mmol})$ suspended in DCM $(4 \mathrm{~mL})$ at $0{ }^{\circ} \mathrm{C}$ was added TFA $(414.5 \mu \mathrm{L}, 5.38 \mathrm{mmol})$. After $1 \mathrm{~h}$ of stirring at $0^{\circ} \mathrm{C}$, the solvent and excess TFA were evaporated. The residue was taken-up in EtOAc and washed with aq. sat $\mathrm{NaHCO}_{3}$ solution. The aqueous phase was extracted 5 times with EtOAc. The combined organics were dried over $\mathrm{Na}_{2} \mathrm{SO}_{4}$, filtered and concentrated under vacuum. The residue was purified by flash chromatography on silica gel (cyclohexane/ EtOA, gradient of $1 / 1$ to $0 / 1$ ) to yield expected product ( $195 \mathrm{mg}$, $75 \%)$ as a white solid: $\mathrm{LCMS}\left(t_{\mathrm{R}}=3.10 \mathrm{~min}\right.$, purity $\left.=93 \%\right) \mathrm{ESI}^{+} \mathrm{m} / \mathrm{z}$ $482.2(\mathrm{M}+\mathrm{H})^{+} ;{ }^{1} \mathrm{H}$ NMR $\left(300 \mathrm{MHz}\right.$, DMSO- $\left.d_{6}\right) \delta 9.02-9.14(\mathrm{~m}, 1 \mathrm{H})$. $8.50(\mathrm{~s}, 1 \mathrm{H}), 8.09-8.15(\mathrm{~m}, 0.6 \mathrm{H}) 7.90-8.09(\mathrm{~m}, 4 \mathrm{H}), 7.56-7.69(\mathrm{~m}$, $2 \mathrm{H}), 5.53(\mathrm{~d}, J=5.1 \mathrm{~Hz}, 0.3 \mathrm{H}), 5.30-5.35(\mathrm{~m}, 0.5 \mathrm{H}), 4.80-5.12(\mathrm{~m}$, $1.7 \mathrm{H}), 4.50-4.65(\mathrm{~m}, 0.3 \mathrm{H}), 4.05-4.15(\mathrm{~m}, 1 \mathrm{H}), 3.52-3.80(\mathrm{~m}, 2 \mathrm{H})$, $2.54-3.06(\mathrm{~m}, 3 \mathrm{H}), 2.00-2.50(\mathrm{~m}, 3 \mathrm{H}), 1.24-1.39(\mathrm{~m}, 3 \mathrm{H}), 1.10-1.20$ $(\mathrm{m}, 3 \mathrm{H})$.

4.2.7. $\mathrm{N}-((3 R)-1-((2 S)-1-(((3 S)-2-h y d r o x y-5-o x o t e t r a h y d r o-f u r a n-$ 3-yl)amino)-1-oxopropan-2-yl)-2,6-dioxopiperidin-3-yl)-2naphthamide (2)

To a suspension of $N-((3 R)-1-((2 S)-1-(((3 S)-2-$ ethoxy-5oxotetrahydrofuran-3-yl)amino)-1-oxopropan-2-yl)-2,6-dioxopiperidin-3-yl)-2-naphthamide (190 mg, $394.6 \mu \mathrm{mol})$ in acetonitrile $(2.5 \mathrm{~mL})$ was added aq. $2 \mathrm{~N} \mathrm{HCl}(1.97 \mathrm{~mL}, 3.95 \mathrm{mmol}, 10$ eq. $)$. After $6 \mathrm{~h}$ of stirring at room temperature, LCMS monitoring showed complete conversion. The reaction mixture was diluted with EtOAc. The phases were separated and the aqueous phase was extracted with EtOAc $(5 \times 6 \mathrm{~mL})$. The combined organics were then dried over $\mathrm{Na}_{2} \mathrm{SO}_{4}$, filtered and concentrated under reduced pressure. The residue $(173 \mathrm{mg}$ ) was purified by preparative HPLC (MeCN/ $\mathrm{H}_{2} \mathrm{O} / \mathrm{HCO}_{2} \mathrm{H} 10 \mathrm{mM}$ ). The pure fractions were frozen and lyophilised to yield expected product $2(93 \mathrm{mg}, 52 \%)$ as a white solid: LCMS $\left(t_{\mathrm{R}}=4.06 \mathrm{~min}\right.$, purity $\left.=97 \%\right) \mathrm{ESI}^{+} \mathrm{m} / z \quad 454.5(\mathrm{M}+\mathrm{H})^{+}:{ }^{1} \mathrm{H}$ NMR $\left(300 \mathrm{MHz}\right.$, DMSO- $\left.d_{6}\right) \delta 8.95-9.15(\mathrm{~m}, 1 \mathrm{H}), 8.51(\mathrm{~s}, 1 \mathrm{H})$, 7.93-8.10 (m, 4H), $7.72(\mathrm{~m}, 1 \mathrm{H}), 7.56-7.68(\mathrm{~m}, 2 \mathrm{H}), 5.50(\mathrm{~m}, 1 \mathrm{H})$, 
4.76-5.15 (m, 2H), 3.95-4.25 (m, 1H), 2.67-3.02 (m, 3H), $2.38-2.50(\mathrm{~m}, 1 \mathrm{H}), 2.15-2.37(\mathrm{~m}, 1 \mathrm{H}), 2.20-2.50(\mathrm{~m}, 1 \mathrm{H}), 1.95-2.10$ $(\mathrm{m}, 1 \mathrm{H}), 1.31 \& 1.36(2 \mathrm{~d}, J=6.9 \mathrm{~Hz}, 3 \mathrm{H})$.

\section{3. $N-(3-(1-(((3 S)-2-H y d r o x y-5-o x o t e t r a h y d r o f u r a n-3-y l)$} carbamoyl)cyclopropyl)-1-isopropyl-2,4-dioxo-1,2,3,4tetrahydropyrimidin-5-yl)-2-methyl-4-(naphthalen-2-ylamino) benzamide (16) was prepared in 12 steps

\subsubsection{1-Ureidocyclopropane-1-carboxylic acid (18)}

A suspension of 1-aminocyclopropane-1-carboxylic acid (17, $120 \mathrm{~g}, 1.19 \mathrm{~mol}, 1.0 \mathrm{eq}$.) and potassium cyanate ( $150 \mathrm{~g}, 1.85 \mathrm{~mol}, 1.56$ eq.) in water $(500 \mathrm{~mL})$ was heated to reflux temperature during $3 \mathrm{~h}$. Upon completion the mixture was cooled to $3{ }^{\circ} \mathrm{C}$ and aqueous hydrochloric acid 37\% (200 mL, 2.03 eq.) was slowly added (CARE: vigorous $\mathrm{CO}_{2}$ gas release) to adjust to $\mathrm{pH} 2$ and a white precipitate appeared. The white precipitate was filtered, washed with iced water $(250 \mathrm{~mL})$ acetone $(3 \times 255 \mathrm{~mL})$ and dried. 1Ureidocyclopropane-1-carboxylic acid (18, 133.7 g, 78\%) was obtained as a white solid: LCMS $\left(t_{\mathrm{R}}=0.22 \mathrm{~min}\right.$, purity $\left.=100 \%\right) \mathrm{ESI}^{+} \mathrm{m} /$ $z 144.94(\mathrm{M}+\mathrm{H})^{+}, 166.99(\mathrm{M}+\mathrm{Na})^{+} ;{ }^{1} \mathrm{H}$ NMR $\left(400 \mathrm{MHz}\right.$, DMSO-d $\left.d_{6}\right)$ $\delta 6.61(\mathrm{~s}, 1 \mathrm{H}), 5.55(\mathrm{~s}, 2 \mathrm{H}), 1.26(\mathrm{q}, J=4.2 \mathrm{~Hz}, 2 \mathrm{H}), 0.93(\mathrm{q}, J=4.2 \mathrm{~Hz}$, $2 \mathrm{H}) ;{ }^{13} \mathrm{C}$ NMR $\left(101 \mathrm{MHz}, \mathrm{DMSO}-d_{6}\right) \delta 173.80,158.71,51.96,17.47$.

\subsubsection{Allyl 1-ureidocyclopropane-1-carboxylate (19)}

To a suspension of 1-ureidocyclopropane-1-carboxylic acid (18, $122 \mathrm{~g}, 0.85 \mathrm{~mol}, 1$ eq.) in a tetrahydrofuran $(1 \mathrm{~L}) /$ water $(100 \mathrm{~mL})$ mixture was added $N, N$-diisopropylethylamine $(175 \mathrm{~mL}, 1.02 \mathrm{~mol}$, 1.2 eq.) and allyl bromide ( $88 \mathrm{~mL}, 1.02 \mathrm{~mol}, 1.2$ eq.). The reaction mixture was heated to reflux during $18 \mathrm{~h}$. Upon completion mixture was cooled to room temperature then saturated aqueous ammonium chloride solution $(300 \mathrm{~mL})$ and ethyl acetate $(1 \mathrm{~L})$ were added. The aqueous phase was extracted with ethyl acetate $(2 \times 1 \mathrm{~L})$ and 2 methyltetrahydrofuran $(5 \times 1 \mathrm{~L})$. The organic phases were gathered and concentrated under reduced pressure to $1.5 \mathrm{~L}$ liter. The organic phase was washed with saturated aqueous ammonium chloride solution $(2 \times 100 \mathrm{~mL})$, dried over $\mathrm{MgSO}_{4}$, filtered, and concentrated under reduced pressure. The white solid obtained was triturated in diisopropyl ether $(1.25 \mathrm{~L})$, filtered, washed with diisopropyl ether and dried. Allyl 1-ureidocyclopropane-1-carboxylate $(19,67.8 \mathrm{~g}$, $68 \%$ ) was obtained as a white solid: LCMS $\left(t_{R}=0.50 \mathrm{~min}\right.$, purity $=90 \%) \mathrm{ESI}^{+} \mathrm{m} / \mathrm{z} 184.98(\mathrm{M}+\mathrm{H})^{+} ;{ }^{1} \mathrm{H}$ NMR (400 MHz, DMSO- $\left.d_{6}\right)$ $\delta 6.66(\mathrm{~s}, 1 \mathrm{H}), 5.88(\mathrm{ddt}, J=17.3,10.3,5.0 \mathrm{~Hz}, 1 \mathrm{H}), 5.58(\mathrm{~s}, 2 \mathrm{H})$, 5.39-5.11 (m, 2H), $4.53(\mathrm{dt}, J=5.0,1.7 \mathrm{~Hz}, 2 \mathrm{H}), 1.33(\mathrm{q}, J=4.3 \mathrm{~Hz}$, $2 \mathrm{H}), 1.02(\mathrm{q}, J=4.4 \mathrm{~Hz}, 2 \mathrm{H}) ;{ }^{13} \mathrm{C}$ NMR (101 MHz, DMSO-d $\left.d_{6}\right) \delta 172.97$, 158.73, 132.65, 117.10, 64.66, 33.49, 17.51; HRMS: $(\mathrm{M}+\mathrm{H})^{+}$calculated for $\mathrm{C}_{8} \mathrm{H}_{12} \mathrm{~N}_{2} \mathrm{O}_{3}$ 185.0848; found 185.0921 .

\subsubsection{Allyl (E/Z) 1-(3-(3-ethoxy-2-nitro-3-oxoprop-1-en-1-yl) ureido)cyclopropane-1-carboxylate (20)}

To a suspension of allyl 1-ureidocyclopropane-1-carboxylate $(19,95.0 \mathrm{~g}, 0.52 \mathrm{~mol}, 1 \mathrm{eq}$.) in toluene $(500 \mathrm{~mL})$ were successively added ethyl nitroacetate $(69 \mathrm{~mL}, 0.62 \mathrm{~mol}, 1.2 \mathrm{eq})$ and triethyl orthoformate ( $103 \mathrm{~mL}, 0.62 \mathrm{~mol}, 1.2 \mathrm{eq}$.). The reaction mixture was heated to reflux during $15 \mathrm{~h}$. To complete the reaction 2 further additions of ethyl nitroacetate $(2 \times 12 \mathrm{~mL}, 2 \times 0.2 \mathrm{eq})$ and triethyl orthoformate $(2 \times 17 \mathrm{~mL}, 2 \times 0.2$ eq. $)$ were added. Upon reaction completion heptane $(1 \mathrm{~L})$ was slowly added at reflux temperature. The heating was stopped and the reaction mixture slowly crystallised. The yellow solid was filtered, washed with heptane and dried. Allyl 1-(3-(3-ethoxy-2-nitro-3-oxoprop-1-en-1-yl)ureido)-cyclopropane-1-carboxylate $(\mathbf{2 0}, 135 \mathrm{~g}, 79 \%)$ was obtained as a yellow solid as $\sim 7 / 3$ mixture of $E / Z$ isomers: LCMS $\left(t_{\mathrm{R}}=1.04 \mathrm{~min}\right.$, purity $=100 \%) \mathrm{ESI}^{+} \mathrm{m} / \mathrm{z} 328.00(\mathrm{M}+\mathrm{H})^{+} ;{ }^{1} \mathrm{H} \quad \mathrm{NMR}(400 \mathrm{MHz}$, DMSO- $\left._{6}\right) \delta 10.81(\mathrm{~d}, J=13.2 \mathrm{~Hz}, 0.7 \mathrm{H}), 10.29(\mathrm{~d}, J=12.7 \mathrm{~Hz}, 0.3 \mathrm{H})$, $8.93(\mathrm{~s}, 0.3 \mathrm{H}), 8.87(\mathrm{~d}, J=12.7 \mathrm{~Hz}, 0.3 \mathrm{H}), 8.76(\mathrm{~s}, 0.7 \mathrm{H}), 8.50(\mathrm{~d}$, $J=13.3 \mathrm{~Hz}, 0.7 \mathrm{H}), 5.89(\mathrm{~m}, 1 \mathrm{H}), 5.28(\mathrm{dq}, J=17.2,1.7 \mathrm{~Hz}, 1 \mathrm{H}), 5.21$ $(\mathrm{dq}, J=10.5,1.5 \mathrm{~Hz}, 1 \mathrm{H}), 4.62-4.50(\mathrm{~m}, 2 \mathrm{H}), 4.33(\mathrm{q}, J=7.1 \mathrm{~Hz}$, $0.8 \mathrm{H}), 4.23(\mathrm{q}, J=7.1 \mathrm{~Hz}, 1.2 \mathrm{H}), 1.48(\mathrm{q}, J=4.7 \mathrm{~Hz}, 2 \mathrm{H}), 1.37-1.16(\mathrm{~m}$, $5 \mathrm{H}) ;{ }^{13} \mathrm{C}$ NMR (101 MHz, DMSO- $\left.d_{6}\right) \delta 171.24,159.93,152.08,141.43$, 132.42, 119.58, 117.53, 65.24, 61.21, 33.60, 17.11, 14.07; HRMS: $(\mathrm{M}+\mathrm{H})^{+}$calculated for $\mathrm{C}_{13} \mathrm{H}_{17} \mathrm{~N}_{3} \mathrm{O}_{7} 328.1066$; found 328.1137.

\subsubsection{Allyl 1-(5-nitro-2,6-dioxo-3,6-dihydropyrimidin-1(2H)-yl) cyclopropane-1-carboxylate (21)}

A suspension of allyl 1-(3-(3-ethoxy-2-nitro-3-oxoprop-1-en-1yl)ureido)cyclopropane-1-carboxylate $(\mathbf{2 0}, 135 \mathrm{~g}, 0.41 \mathrm{~mol}, 1$ eq.) and cesium carbonate $(296 \mathrm{~g}, 0.91 \mathrm{~mol}, 2.2 \mathrm{eq}$.) in acetonitrile $(1.35 \mathrm{~L})$ was heated under reflux for $30 \mathrm{~min}$. The reaction mixture was cooled to room temperature and diisopropyl ether $(500 \mathrm{~mL})$ was added. The organic phase was discarded and the basic aqueous phase was cooled to $5{ }^{\circ} \mathrm{C}$ and $\mathrm{pH}$ was adjusted to 2 with aqueous hydrochloric acid 37\% [CARE: Vigorous release of $\mathrm{CO}_{2}(\mathrm{~g})$ ]. The acidic aqueous phase was extracted with dichloromethane $(2 \times 1 \mathrm{~L})$ and the organic phase was dried over magnesium sulphate and evaporated under reduce pressure. A brown oil was obtained and precipitated in an isopropanol/diisopropyl ether mixture. The solid was filtered, washed with diisopropyl ether $(500 \mathrm{~mL})$ and dried. Allyl 1-(5-nitro-2,6-dioxo-3,6-dihydropyrimidin-1(2H)-yl)cyclopropane-1-carboxylate $(\mathbf{2 1}, 83.1 \mathrm{~g}, 73 \%)$ was obtained as an orange solid: LCMS $\left(t_{\mathrm{R}}=0.83 \mathrm{~min}\right.$, purity $\left.=100 \%\right) \mathrm{ESI}^{+} \mathrm{m} / z 280.07(\mathrm{M}-\mathrm{H})^{-}$; ${ }^{1} \mathrm{H}$ NMR (400 MHz, DMSO- $\left.d_{6}\right) \delta 12.65(\mathrm{~s}, 1 \mathrm{H}), 8.97(\mathrm{~s}, 1 \mathrm{H}), 5.85(\mathrm{~m}$, $1 \mathrm{H}), 5.28-5.13(\mathrm{~m}, 2 \mathrm{H}), 4.58(\mathrm{~m}, 2 \mathrm{H}), 1.83-1.72(\mathrm{~m}, 2 \mathrm{H}), 1.41(\mathrm{~m}, 2 \mathrm{H})$.

\subsubsection{Allyl 1-(3-isopropyl-5-nitro-2,6-dioxo-3,6-dihydro-}

pyrimidin-1(2H)-yl)cyclopropane-1-carboxylate (22)

To a stirred suspension of 1-(5-nitro-2,6-dioxo-3,6-dihydro-2Hpyrimidin-1-yl)-cyclopropanecarboxylic acid allyl ester (21, $4.58 \mathrm{~g}$, $16.3 \mathrm{mmol}, 1.00$ eq.) and $\mathrm{K}_{2} \mathrm{CO}_{3}(3.38 \mathrm{~g}, 0.02 \mathrm{~mol}, 1.50$ eq.) in $\mathrm{DMF}$ $(91.6 \mathrm{~mL})$ at r.t., was added 2-iodopropane $(8.15 \mathrm{~mL}, 81.5 \mathrm{mmol}, 5.00$ eq.) and the suspension was stirred at r.t. for $24 \mathrm{~h}$. The reaction mixture was slowly added to water and the product was extracted in EtOAc $(3 \times 100 \mathrm{~mL})$. The organic phases were combined, washed with water $(3 \times 50 \mathrm{~mL})$, dried over $\mathrm{MgSO}_{4}$ and concentrated to dryness. The residue was purified by flash chromatography (silica gel, gradient 0-20\% EtOAc in heptane) to afford 1-(3-isopropyl-5-nitro2,6-dioxo-3,6-dihydro-2H-pyrimidin-1-yl)-cyclopropanecarboxylic acid allyl ester $(\mathbf{2 2}, 2.41 \mathrm{~g}, 46 \%)$ as a white solid: $\mathrm{LCMS}\left(t_{\mathrm{R}}=1.04 \mathrm{~min}\right.$, purity $=99 \%), \mathrm{ESI}^{+} \mathrm{m} / z 324.5(\mathrm{M}+\mathrm{H})^{+} ;{ }^{1} \mathrm{H}$ NMR $\left(400 \mathrm{MHz}\right.$, DMSO- $\left.d_{6}\right)$ $\delta 9.13(\mathrm{~s}, 1 \mathrm{H}), 5.97-5.69(\mathrm{~m}, 1 \mathrm{H}), 5.31-5.05(\mathrm{~m}, 2 \mathrm{H}), 4.79-4.68(\mathrm{~m}$, $1 \mathrm{H}), 4.58(\mathrm{ddt}, J=8.5,4.9,1.7 \mathrm{~Hz}, 2 \mathrm{H}), 1.91-1.59(\mathrm{~m}, 2 \mathrm{H}), 1.50-1.10(\mathrm{~m}$, $8 \mathrm{H})$.

\subsubsection{Allyl 1-(5-amino-3-isopropyl-2,6-dioxo-3,6-dihydro- pyrimidin-1(2H)-yl)cyclopropane-1-carboxylate (23)}

A stirred suspension of 1-(3-isopropyl-5-nitro-2,6-dioxo-3,6dihydro-2H-pyrimidin-1-yl)-cyclopropanecarboxylic acid allyl ester (22, $2.41 \mathrm{~g}, 7.45 \mathrm{mmol}, 1.00$ eq.), ammonium chloride $(0.20 \mathrm{~g}$, $3.73 \mathrm{mmol}, 0.50 \mathrm{eq}$.), iron ( $1.67 \mathrm{~g}, 29.8 \mathrm{mmol}, 4.00 \mathrm{eq}$.) in a mixture of EtOH $(14.5 \mathrm{~mL})$, THF $(14.5 \mathrm{~mL})$ and water $(14.5 \mathrm{~mL})$ was heated at $70{ }^{\circ} \mathrm{C}$ for $1 \mathrm{~h}$. The reaction mixture was cooled to r.t., filtered through a pad of Celite and washed with EtOH $(5 \times 5 \mathrm{~mL})$. The filtrate was concentrated to dryness to afford 1-(5-amino-3-isopropyl-2,6dioxo-3,6-dihydro-2H-pyrimidin-1-yl)-cyclopropanecarboxylic acid allyl ester $(23,2.31 \mathrm{~g}, 98 \%)$ as an orange gum: LCMS $\left(t_{\mathrm{R}}=0.99 \mathrm{~min}\right.$, purity $=100 \%), \mathrm{ESI}^{+} \mathrm{m} / \mathrm{z} 294.16(\mathrm{M}+\mathrm{H})^{+} ;{ }^{1} \mathrm{H}$ NMR $(400 \mathrm{MHz}$, DMSO- $\left.d_{6}\right) \delta 6.96(\mathrm{~s}, 1 \mathrm{H}), 5.92-5.75(\mathrm{~m}, 1 \mathrm{H}), 5.28-5.08(\mathrm{~m}, 2 \mathrm{H})$, 4.79-4.63 (m,1H), 4.61-4.42 (m, 2H), 4.09 (s, 2H), 1.83-1.61 (m, 2H), $1.41-1.30(\mathrm{~m}, 2 \mathrm{H}), 1.25-1.15(\mathrm{~m}, 6 \mathrm{H}) ;{ }^{13} \mathrm{C}$ NMR (101 MHz, DMSO-d $\left.\mathrm{d}_{6}\right)$ $\delta$ 170.51, 160.39, 149.39, 132.32, 122.42, 116.63, 115.19, 64.86, 47.04, 
$39.05,35.46,20.92,20.48,19.53,19.33$; HRMS $(\mathrm{M}+\mathrm{H})^{+}$calculated for $\mathrm{C}_{14} \mathrm{H}_{19} \mathrm{~N}_{3} \mathrm{O}_{4}$ 294.1375; found 294.1448.

\subsubsection{2-Methyl-4-(naphthalen-2-ylamino)benzoic acid (24)}

Step 1: A stirred solution of 2-aminonaphthalene (2.38 g, $16.63 \mathrm{mmol}, 1.00$ eq.), 4-bromo-2-methyl-benzoic acid methyl ester ( $4.00 \mathrm{~g}, 17.5 \mathrm{mmol}, 1.05 \mathrm{eq}$.), palladium(II) acetate (373 mg, $1.66 \mathrm{mmol}$, 0.10 eq.), 4,5-bis(diphenylphosphino)-9,9-dimethylxanthene (1.92 g, $3.33 \mathrm{mmol}, 0.20$ eq.) and cesium carbonate $(7.04 \mathrm{~g}, 21.6 \mathrm{mmol}, 1.30$ eq.) in 1,4-dioxanne (38.1 mL) was degased and purged with nitrogen then heated at $120^{\circ} \mathrm{C}$ under microwave irradiation for $30 \mathrm{~min}$. The reaction was concentrated to dryness and partitioned between DCM $(50 \mathrm{~mL})$ and water $(5 \mathrm{~mL})$. The organic phase was retained and the aqueous phase was washed with $\mathrm{DCM}(3 \times 5 \mathrm{~mL})$. The organic phases were combined, washed with water $(10 \mathrm{~mL})$, dried over $\mathrm{MgSO}_{4}$ and concentrated to dryness to afford a pale yellow residue. The residue was triturated with a mixture of DCM and heptane to afford a solid that was collected by filtration and dried to a constant weight to afford 2-methyl-4-(naphthalen-2-ylamino)-benzoic acid methyl ester $(4.30 \mathrm{~g}, 89 \%)$ as a pale brown solid: LCMS $\left(t_{\mathrm{R}}=1.35 \mathrm{~min}\right.$, purity $=100 \%), \mathrm{ESI}^{+} \mathrm{m} / \mathrm{z} 292.01(\mathrm{M}+\mathrm{H})^{+} ;{ }^{1} \mathrm{H} \mathrm{NMR}\left(400 \mathrm{MHz}\right.$, DMSO- $\left.d_{6}\right)$ $\delta 8.89(\mathrm{~s}, 1 \mathrm{H}), 7.93-7.75(\mathrm{~m}, 5 \mathrm{H}), 7.63(\mathrm{~d}, J=2.2 \mathrm{~Hz}, 1 \mathrm{H}), 7.44$ (ddd, $J=8.2,6.8,1.3 \mathrm{~Hz}, 1 \mathrm{H}), 7.40-7.29(\mathrm{~m}, 2 \mathrm{H}), 7.05(\mathrm{dd}, J=8.6,2.4 \mathrm{~Hz}, 1 \mathrm{H})$ $7.00(\mathrm{~d}, J=2.3 \mathrm{~Hz}, 1 \mathrm{H}), 3.78$ (s, 3H), 2.50 (s, 3H under DMSO signal).

Step 2: To a stirred solution of 2-methyl-4-(naphthalen-2ylamino)-benzoic acid methyl ester $(4.00 \mathrm{~g}, 13.7 \mathrm{mmol}, 1.00 \mathrm{eq}$.) in a mixture of THF $(80.0 \mathrm{~mL})$ and $\mathrm{MeOH}(20.0 \mathrm{~mL})$, was added lithium hydroxide $(1.0 \mathrm{M}, 41.2 \mathrm{~mL}, 41.2 \mathrm{mmol}, 3.00$ eq.). The reaction mixture was stirred at $50{ }^{\circ} \mathrm{C}$ overnight, concentrated to a minimum volume and the residue was partitioned between DCM $(30 \mathrm{~mL})$ and water $(5 \mathrm{~mL})$. The $\mathrm{pH}$ of the aqueous phase was adjusted to 1 by the addition of $\mathrm{HCl}(\mathrm{aq})(1.0 \mathrm{M})$ resulting in a thick precipitate that was collected by filtration and dried to a constant weight to afford 2-methyl-4-(naphthalen-2-ylamino)benzoic acid $(24,3.50 \mathrm{~g}, 92 \%)$ as a beige solid: LCMS $\left(t_{\mathrm{R}}=1.20 \mathrm{~min}\right.$, purity $=100 \%), \mathrm{ESI}^{+} \mathrm{m} / \mathrm{z} 278.02(\mathrm{M}+\mathrm{H})^{+} ;{ }^{1} \mathrm{H}$ NMR $(400 \mathrm{MHz}$, DMSO-d $\left.d_{6}\right) \delta 12.19(\mathrm{br} \mathrm{s}, 1 \mathrm{H}), 8.82(\mathrm{~s}, 1 \mathrm{H}), 7.98-7.72(\mathrm{~m}, 4 \mathrm{H}), 7.61(\mathrm{~d}$, $J=2.3 \mathrm{~Hz}, 1 \mathrm{H}), 7.50-7.25(\mathrm{~m}, 3 \mathrm{H}), 7.10-6.90(\mathrm{~m}, 2 \mathrm{H}), 2.51(\mathrm{~s}, 3 \mathrm{H}$ hidden by DMSO signal); ${ }^{13} \mathrm{C}$ NMR $\left(101 \mathrm{MHz}\right.$, DMSO- $\left.d_{6}\right) \delta 168.07$, 146.95, 141.82, 139.60, 134.19, 132.88, 129.01, 127.51, 128.96, 126.66, 126.43, 123.72, 120.18, 117.95, 112.68, 112.31, 22.25; HRMS: $(\mathrm{M}+\mathrm{H})^{+}$ calculated for $\mathrm{C}_{18} \mathrm{H}_{15} \mathrm{NO}_{2} 278.1102$; found 278.1172 .

\subsubsection{Allyl 1-(3-isopropyl-5-(2-methyl-4-(naphthalen-2-ylamino) benzamido)-2,6-dioxo-3,6-dihydro-pyrimidin-1(2H)-yl) cyclopropane-1-carboxylate}

To a stirred solution of 2-methyl-4-(naphthalen-2-ylamino)benzoic acid (24, $520 \mathrm{mg}, 1.88 \mathrm{mmol}, 1.10$ eq.) and $\mathrm{N}$-methylmorpholine ( $375 \mu \mathrm{L}, 3.41 \mathrm{mmol}, 2.00$ eq.) in DMF ( $10.0 \mathrm{~mL})$ at r.t., was added COMU (\{[1-cyano-1-ethoxycarbonyl-meth-( $Z$ )-ylideneaminooxy]-morpholin-4-yl-methylene\}-dimethyl-ammonium hexafluorophosphate) ( $876 \mathrm{mg}, 2.05 \mathrm{mmol}, 1.20$ eq.) and the reaction mixture was stirred at r.t. for $30 \mathrm{~min}$ 1-(5-amino-3-isopropyl2,6-dioxo-3,6-dihydro-2H-pyrimidin-1-yl)-cyclo-

propanecarboxylic acid allyl ester (500 $\mathrm{mg}, 1.70 \mathrm{mmol}, 1.00$ eq.) in DMF $(5.00 \mathrm{~mL})$ was added and the reaction mixture was heated at $50^{\circ} \mathrm{C}$ for $48 \mathrm{~h}$. The reaction mixture was treated with a saturated solution of $\mathrm{NaHCO}_{3}(\mathrm{aq})$ and the product extracted with EtOAc $(3 \times 30 \mathrm{~mL})$. The organic phases were combined and washed with water $\left(3 \times 10 \mathrm{~mL}\right.$ ), dried over $\mathrm{MgSO}_{4}$ and concentrated to dryness. The crude product was purified by flash chromatography (silica gel, gradient of $0-100 \%$ EtOAc in heptane) to afford 1-\{3-Isopropyl-5[2-methyl-4-(naphthalen-2-ylamino)-benzoylamino]-2,6-dioxo3,6-dihydro-2H-pyrimidin-1-yl\}-cyclopropanecarboxylic acid allyl ester ( $850 \mathrm{mg}, 90 \%)$ as a pale yellow solid: LCMS $\left(t_{\mathrm{R}}=1.43 \mathrm{~min}\right.$, purity $=100 \%), \mathrm{ESI}^{+} \mathrm{m} / \mathrm{z} 533.22(\mathrm{M}+\mathrm{H})^{+} ;{ }^{1} \mathrm{H}$ NMR $(400 \mathrm{MHz}$, DMSO-d $\left.d_{6}\right) \delta 8.98(\mathrm{~s}, 1 \mathrm{H}), 8.69(\mathrm{~s}, 1 \mathrm{H}), 8.30(\mathrm{~s}, 1 \mathrm{H}), 7.93-7.68(\mathrm{~m}, 4 \mathrm{H})$, $7.57(\mathrm{~d}, J=2.2 \mathrm{~Hz}, 1 \mathrm{H}), 7.51(\mathrm{~d}, J=8.4 \mathrm{~Hz}, 1 \mathrm{H}), 7.42(\mathrm{ddd}, J=8.2,6.8$, $1.4 \mathrm{~Hz}, 1 \mathrm{H}$ ), 7.37-7.28 ( $\mathrm{m}, 2 \mathrm{H}), 7.10-6.99$ ( $\mathrm{m}, 2 \mathrm{H}), 5.85$ (ddt, $J=17.3$, $10.7,4.8 \mathrm{~Hz}, 1 \mathrm{H}), 5.33-5.01(\mathrm{~m}, 2 \mathrm{H}), 4.77(\mathrm{~h}, J=6.7 \mathrm{~Hz}, 1 \mathrm{H})$, 4.70-4.43 (m, 2H), $2.43(\mathrm{~s}, 3 \mathrm{H}), 1.84-1.64(\mathrm{~m}, 2 \mathrm{H}), 1.55-1.20(\mathrm{~m}$, $8 \mathrm{H}) ;{ }^{13} \mathrm{C}$ NMR (DMSO- $\left.d_{6}\right) \delta 170.24,159.94,149.89,145.30,140.18$, $138.35,134.26,132.27,131.18,129.61,128.98,128.70,127.50,126.50$, 126.40, 126.19, 123.44, 120.58, 118.16, 116.88, 113.03, 112.76, 111.48, $65.04,54.95,48.38,35.67,20.93,20.58,19.48,19.22$; HRMS: $(\mathrm{M}+\mathrm{H})^{+}$calculated for $\mathrm{C}_{32} \mathrm{H}_{32} \mathrm{~N}_{4} \mathrm{O}_{5}$ 553.2372; found 553.2443.

4.3.9. 1-(3-Isopropyl-5-(2-methyl-4-(naphthalen-2-ylamino)benzamido)-2,6-dioxo-3,6-dihydro-pyrimidin-1(2H)-yl) cyclopropane-1-carboxylic acid (25)

A stirred solution of 1-\{3-isopropyl-5-[2-methyl-4-(naphthalen-2-ylamino)-benzoylamino]-2,6-dioxo-3,6-dihydro-2H-pyrimidin-1-yl\}-cyclopropanecarboxylic acid allyl ester $(840 \mathrm{mg}$, $1.52 \mathrm{mmol}, \quad 1.00$ eq.), 1,3-dimethylbarbituric acid $(380 \mathrm{mg}$, $2.43 \mathrm{mmol}, 1.60$ eq.) in DCM ( $42 \mathrm{~mL})$ was degassed and purged with nitrogen and tetrakis(triphenylphosphine)palladium $(0)(70.3 \mathrm{mg}$, $0.06 \mathrm{mmol}, 0.04$ eq.) was added. The reaction mixture was stirred at r.t. for $45 \mathrm{~min}$, concentrated and purified by flash chromatography (silica gel, gradient of $0-10 \% \mathrm{MeOH}$ in DCM) to afford $1-\{3-$ isopropyl-5-[2-methyl-4-(naphthalen-2-ylamino)-benzoylamino]2,6-dioxo-3,6-dihydro-2H-pyrimidin-1-yl\}-cyclopropane-carboxylic acid $(\mathbf{2 5}, 470 \mathrm{mg}, 60 \%)$ as a beige solid: $\mathrm{LCMS}\left(t_{\mathrm{R}}=1.23 \mathrm{~min}\right.$, purity $=99 \%), \mathrm{ESI}^{+} \mathrm{m} / \mathrm{z} 513.14(\mathrm{M}+\mathrm{H})^{+} ;{ }^{1} \mathrm{H} \quad \mathrm{NMR}(400 \mathrm{MHz}$, DMSO- $\left.d_{6}\right) \delta{ }^{1} \mathrm{H}$ NMR $\left(400 \mathrm{MHz}\right.$, DMSO- $\left.d_{6}\right) \delta 12.6$ (br s, $\left.1 \mathrm{H}\right), 8.93(\mathrm{~s}$, $1 \mathrm{H}), 8.69(\mathrm{~s}, 1 \mathrm{H}), 8.28(\mathrm{~s}, 1 \mathrm{H}), 7.92-7.70(\mathrm{~m}, 3 \mathrm{H}), 7.57(\mathrm{~d}, J=2.2 \mathrm{~Hz}$, $1 \mathrm{H}), 7.51$ (d, $J=8.3 \mathrm{~Hz}, 1 \mathrm{H}), 7.42$ (ddd, $J=8.2,6.8,1.3 \mathrm{~Hz}, 1 \mathrm{H}$ ), 7.37-7.28 (m, 2H), 7.09-7.00 (m, 2H), 4.96-4.49 (m, 1H), $2.44(\mathrm{~s}$, $3 \mathrm{H}), 1.67(\mathrm{~m}, 2 \mathrm{H}), 1.45-1.20(\mathrm{~m}, 8 \mathrm{H})$.

\subsubsection{Allyl ((3S)-2-ethoxy-5-oxotetrahydrofuran-3-yl)-carbamate (26)}

To a cooled $\left(4^{\circ} \mathrm{C}\right)$ solution of tert-butyl (S)-3-(((allyloxy) carbonyl)amino)-4,4-diethoxybutanoate $(6,5.0 \mathrm{~g}, 15.1 \mathrm{mmol})$ in DCM $(20 \mathrm{~mL})$ was added trifluoroacetic acid $(11.6 \mathrm{~mL}, 151 \mathrm{mmol})$. After $30 \mathrm{~min}$ of stirring at room temperature, the mixture was concentrated under vacuum, then co-evaporated with EtOAc. Purification of the residue by flash chromatography on silica gel (cyclohexane-EtOAc, gradient of 95/5 to 6/4) afforded the title compound $(2.45 \mathrm{~g}, 71 \%)$ as a pink oil as a $1: 1.2$ mixture of two diastereoisomers): $\mathrm{LCMS} \mathrm{ESI}^{+} \mathrm{m} / z 229.9(\mathrm{M}+\mathrm{H})^{+} ;{ }^{1} \mathrm{H}$ NMR $\left(\mathrm{CDCl}_{3}\right.$, $500 \mathrm{MHz}) \delta 5.97-5.85(\mathrm{~m}, 1 \mathrm{H}), 5.44(\mathrm{~d}, J=5.0 \mathrm{~Hz}, 0.5 \mathrm{H}), 5.40(\mathrm{br} \mathrm{s}$, $0.5 \mathrm{H}), 5.36-5.22(\mathrm{~m}, 2.5 \mathrm{H}), 5.00(\mathrm{br} \mathrm{s}, 0.5 \mathrm{H}), 4.62-4.57(\mathrm{~m}, 2 \mathrm{H})$, $4.57-4.52$ (br s, $0.5 \mathrm{H}), 4.20$ (br t, $J=6.5 \mathrm{~Hz}, 0.5 \mathrm{H}), 3.95-3.83(\mathrm{~m}$, $1 \mathrm{H}), 3.69-3.62(\mathrm{~m}, 1 \mathrm{H}), 3.01(\mathrm{dd}, J=18.0 \mathrm{~Hz}$ and $7.5 \mathrm{~Hz}, 0.5 \mathrm{H}), 2.84$ (dd, $J=17.0 \mathrm{~Hz}$ and $8.5 \mathrm{~Hz}, 0.5 \mathrm{H}), 2.46(\mathrm{dd}, J=17.0 \mathrm{~Hz}$ and $10.5 \mathrm{~Hz}$, $0.5 \mathrm{H}), 2.39(\mathrm{dd}, J=18.0 \mathrm{~Hz}$ and $1.4 \mathrm{~Hz}, 0.5 \mathrm{H}), 1.26(\mathrm{t}, J=7.0 \mathrm{~Hz}, 1.5 \mathrm{H})$, $1.24(\mathrm{t}, J=7.0 \mathrm{~Hz}, 1.5 \mathrm{H}) ;{ }^{13} \mathrm{C}$ NMR $\left(101 \mathrm{MHz}\right.$, DMSO- $\left.d_{6}\right) \delta 174.71$, 173.78, 155.73, 155.56, 133.58, 117.00, 107.23, 102.14, 65.16, 64.60, 52.85, 49.47, 32.89, 31.30, 14.90; HRMS: $(\mathrm{M}+\mathrm{H})^{+}$calculated for $\mathrm{C}_{10} \mathrm{H}_{15} \mathrm{NO}_{5}$ 229.0950; found 230.1023.

4.3.11. N-(3-(1-(((3S)-2-Ethoxy-5-oxotetrahydrofuran-3-yl)carbamoyl)cyclopropyl)-1-isopropyl-2,4-dioxo-1,2,3,4tetrahydropyrimidin-5-yl)-2-methyl-4-(naphthalen-2-ylamino) benzamide (27)

First Step: To a stirred solution of 1-\{3-isopropyl-5-[2-methyl-4(naphthalen-2-ylamino)-benzoylamino]-2,6-dioxo-3,6-dihydro- $2 \mathrm{H}$ pyrimidin-1-yl\}-cyclopropanecarboxylic acid, (1.39 g, $2.71 \mathrm{mmol}, 1.00$ eq.) and $N$-methyl morpholine ( $358 \mu \mathrm{L}, 3.25 \mathrm{mmol}, 1.20$ eq.) in a mixture of DCM $(13.90 \mathrm{~mL})$ and DMF $(13.90 \mathrm{~mL})$, HATU $(1.24 \mathrm{~g}$, 
$3.25 \mathrm{mmol}, 1.20$ eq.) was added and the reaction mixture was stirred at r.t. for $40 \mathrm{~min}$.

Second Step: In parallel, a solution of ((S)-2-ethoxy-5-oxo-tetrahydro-furan-3-yl)-carbamic acid allyl ester, $(870 \mathrm{mg}, 3.80 \mathrm{mmol}$, 1.40 eq.) et 1,3-dimethylbarbituric acid ( $593 \mathrm{mg}, 3.80 \mathrm{mmol}, 1.40$ eq.) in a mixture of DCM $(9.73 \mathrm{~mL})$ and $\operatorname{DMF}(6.95 \mathrm{~mL})$ at r.t. was degassed and purged with nitrogen then tetrakis(triphenylphosphine)palla$\operatorname{dium}(0)(125 \mathrm{mg} ; 0.11 \mathrm{mmol} ; 0.04$ eq.) was added. The reaction mixture was stirred at room temperature for $1 \mathrm{~h}$.

Third step: The product, (4S)-4-amino-5-ethoxydihydrofuran$2(3 \mathrm{H})$-one, from the Alloc deprotection step was added to the preactivated ester for the first step and the reaction mixture was stirred at room temperature overnight. The reaction mixture was treated with a saturated solution of $\mathrm{NaHCO}_{3}(\mathrm{aq})$ and the product extracted with EtOAc $(2 \times 50 \mathrm{~mL})$. The organic phases were combined and washed with water $(3 \times 10 \mathrm{~mL})$, dried over $\mathrm{MgSO}_{4}$ and concentrated to dryness. The crude product was purified by flash chromatography (silica gel, gradient of $0-5 \% \mathrm{MeOH}$ in DCM) to afford $N-\{3-[1-((S)-2-$ ethoxy-5-oxo-tetrahydro-furan-3-ylcarbamoyl)-cyclopropyl]-1isopropyl-2,4-dioxo-1,2,3,4-tetrahydro-pyrimidin-5-yl\}-2-methyl4-(naphthalen-2-ylamino)-benzamide ( $1.80 \mathrm{~g}$, quant.) as an orange solid (mixture of diastereoisomers): LCMS $\left(t_{\mathrm{R}}=1.27 \mathrm{~min}\right.$, purity $=99 \%), \mathrm{ESI}^{+} \mathrm{m} / z 640.30(\mathrm{M}+\mathrm{H}){ }^{+} ;{ }^{1} \mathrm{H}$ NMR $\left(400 \mathrm{MHz}\right.$, DMSO- $\left.d_{6}\right)$ $\delta 8.93-8.77(\mathrm{~m}, 1 \mathrm{H}), 8.71(\mathrm{~s}, 1 \mathrm{H}), 8.43(\mathrm{~s}, 1 \mathrm{H}), 7.88-7.72(\mathrm{~m}, 3 \mathrm{H})$, $7.67-7.53(\mathrm{~m}, 2 \mathrm{H}), 7.49(\mathrm{t}, J=8.3 \mathrm{~Hz}, 1 \mathrm{H}), 7.43(\mathrm{ddd}, J=8.1,6.8,1.2 \mathrm{~Hz}$, 1H), 7.36-7.27 (m, 2H), 7.12-6.98 (m, 2H), $5.50(\mathrm{~m}, 0.5 \mathrm{H}), 5.22(\mathrm{~m}$, $0.5 \mathrm{H}), 4.60-4.70(\mathrm{~m}, 0.5 \mathrm{H}), 4.10-4.20(\mathrm{~m}, 0.5 \mathrm{H}), 3.70-3.80(\mathrm{~m}, 1 \mathrm{H})$, $3.55-3.65(\mathrm{~m}, 1 \mathrm{H}), 2.80-3.20(\mathrm{~m}, 0.5 \mathrm{H}), 2.72-2.56(\mathrm{~m}, 1.5 \mathrm{H}), 2.44(\mathrm{~s}$, $3 \mathrm{H}), 1.60-1.70(\mathrm{~m}, 2 \mathrm{H}), 1.40-1.26(\mathrm{~m}, 6 \mathrm{H}), 1.10-1.20(\mathrm{~m}, 5 \mathrm{H}) ;{ }^{13} \mathrm{C}$ NMR $\left(101 \mathrm{MHz}\right.$, DMSO- $\left.d_{6}\right) \delta 174.01,170.12,169.94,160.04,167.21$, 149.85, 145.42, 140.12, 138.30, 134.26, 129.47, 128.99, 128.73, 127.51, $126.51,126.41,123.48,120.60,118.21,113.97,112.84,111.56,102.12$, 65.49, 48.10, 37.30, 37.22, 26.89, 21.04, 20.82, 20.57, 19.31; HRMS: $(\mathrm{M}+\mathrm{H})^{+}$calculated for $\mathrm{C}_{35} \mathrm{H}_{37} \mathrm{~N}_{5} \mathrm{O}_{7} 640.2693$; found 640.27563 .

4.3.12. $N$-(3-(1-(((3S)-2-Hydroxy-5-oxotetrahydrofuran-3-yl) carbamoyl)cyclopropyl)-1-isopropyl-2,4-dioxo-1,2,3,4-

tetrahydropyrimidin-5-yl)-2-methyl-4-(naphthalen-2-ylamino) benzamide (16)

To a stirred solution of $N-\{3-[1-((S)-2-e t h o x y-5-0 x o-t e t r a h y d r o-$ furan-3-ylcarbamoyl)-cyclopropyl]-1-isopropyl-2,4-dioxo-1,2,3,4tetrahydro-pyrimidin-5-yl\}-2-methyl-4-(naphthalen-2-ylamino)benzamide (1.72 g, $2.69 \mathrm{mmol}, 1.00 \mathrm{eq}$.) in $\mathrm{MeCN}(34.4 \mathrm{~mL})$ was added $\mathrm{HCl}(\mathrm{aq})(2 \mathrm{M}, 13.4 \mathrm{~mL}, 26.9 \mathrm{mmol}, 10.0$ eq.) and the reaction mixture was stirred at room temperature for $5 \mathrm{~h}$. The volatiles were eliminated under a flow of nitrogen and the residue was treated with a saturated aqueous solution of $\mathrm{NaHCO}_{3}(\mathrm{pH} 8)$ then diluted with EtOAc. The organic phase was discarded and the $\mathrm{pH}$ of the aqueous phase was adjusted to 5 with $\mathrm{AcOH}$. The product was extracted in EtOAc $(3 \times 20 \mathrm{~mL})$, the organic phases were combined, dried over $\mathrm{MgSO}_{4}$ and concentrated to dryness. The resulting beige solid was crystallised from a mixture of EtOAc/toluene and the solid was recuperated by filtration and dried to a constant weight un a vacuum oven at $40^{\circ} \mathrm{C}$ to afford $N$-(3-(1-(((3S)-2-hydroxy-5oxotetrahydrofuran-3-yl)carbamoyl)cyclopropyl)-1-isopropyl-2,4dioxo-1,2,3,4-tetrahydro-pyrimidin-5-yl)-2-methyl-4-(naphthalen-2-ylamino)benzamide $(1.07 \mathrm{~g}, 65 \%)$ as an off-white solid: LCMS $\left(t_{\mathrm{R}}=6.47 \mathrm{~min}\right.$, purity $\left.=99 \%\right), \mathrm{ESI}^{-} \mathrm{m} / z 610.62(\mathrm{M}+\mathrm{H})^{+} ;{ }^{1} \mathrm{H}$ $\operatorname{NMR}\left(400 \mathrm{MHz}\right.$, DMSO- $\left.d_{6}\right) \delta 8.96-8.83(\mathrm{~m}, 1 \mathrm{H}), 8.71(\mathrm{~s}, 1 \mathrm{H}), 8.42(\mathrm{~s}$, $1 \mathrm{H}), 8.21$ ( $\mathrm{s} \mathrm{br}, 1 \mathrm{H}), 7.87-7.73(\mathrm{~m}, 3 \mathrm{H}), 7.58(\mathrm{~d}, J=2.3 \mathrm{~Hz}, 1 \mathrm{H}), 7.50$ $(\mathrm{d}, J=8.4 \mathrm{~Hz}, 1 \mathrm{H}), 7.43$ (ddd, $J=8.2,6.8,1.3 \mathrm{~Hz}, 1 \mathrm{H}), 7.36-7.27(\mathrm{~m}$, $2 \mathrm{H}$ ), 7.07 (dd, $J=8.3,2.3 \mathrm{~Hz}, 1 \mathrm{H}), 7.03(\mathrm{~d}, J=2.3 \mathrm{~Hz}, 1 \mathrm{H}), 5.41$ (s br, $1 \mathrm{H}), 4.78(\mathrm{~m}, 1 \mathrm{H}), 4.15(\mathrm{~s} \mathrm{br}, 1 \mathrm{H}), 2.93(\mathrm{~m}, 1 \mathrm{H}), 2.44(\mathrm{~s}, 3 \mathrm{H}), 2.32(\mathrm{~m}$, $1 \mathrm{H}), 1.65(\mathrm{~m}, 2 \mathrm{H}), 1.32$ (dd, $J=13.1,6.7 \mathrm{~Hz}, 6 \mathrm{H}), 1.16(\mathrm{~m}, 2 \mathrm{H}) ;{ }^{13} \mathrm{C}$ NMR (400 MHz, DMSO-d $\left.{ }_{6}\right) \delta 167.31,160.22,149.87,145.41,140.15$,
138.33, 134.28, 129.53, 129.02, 128.75, 128.48, 127.53, 126.53, $126.44,126.14,123.50,120.61,118.24,113.99,112.86,111.55$, 48.09, 39.07, 37.32, 20.98, 20.84, 20.60, 19.38; HRMS: $(\mathrm{M}+\mathrm{H})^{+}$calculated for $\mathrm{C}_{33} \mathrm{H}_{33} \mathrm{~N}_{5} \mathrm{O}_{7}$ 612.2380; found 612.2376; analytical chiral HPLC conditions (Chiralpak IC $4.6 \mathrm{~mm}^{*} 250 \mathrm{~mm} 5 \mu \mathrm{m}$ column; eluent, $n$ heptane/EtOH/TEA (80/20/0.5); flow rate, $1.0 \mathrm{~mL} / \mathrm{min}$.; run time, $50 \mathrm{~min} ; t_{\mathrm{R}}=12.287 \mathrm{~min}$ ( $S$ enantiomer), $t_{\mathrm{R}}=20.100 \mathrm{~min}$ ( $R$ enantiomer), e.r. $=95: 5(S / R)$.

\subsection{Checking the hydrolysis step for epimerization}

4.4.1. (S)-4-Hydroxy-3-(1-(3-isopropyl-5-(2-methyl-4(naphthalen-2-ylamino)benzamido)-2,6-dioxo-3,6-dihydropyrimidin-1(2H)-yl)cyclopropane-1-carboxamido)butanoic acid (29)

To a stirred solution of $N$-(3-(1-(((3S)-2-hydroxy-5oxotetrahydrofuran-3-yl)carbamoyl)cyclopropyl)-1-isopropyl-2,4dioxo-1,2,3,4-tetrahydropyrimidin-5-yl)-2-methyl-4-(naphthalen2-ylamino)benzamide $(\mathbf{1 6}, 85.0 \mathrm{mg}, 0.14 \mathrm{mmol})$ in $\mathrm{MeOH}(850 \mu \mathrm{L})$ was added portionwise $\mathrm{NaBH}_{4}(10.5 \mathrm{mg}, 0.28 \mathrm{mmol})$ at $0{ }^{\circ} \mathrm{C}$ and the reaction mixture was stirred at r.t. for $3 \mathrm{~h} 30 \mathrm{~min}$. The reaction mixture was concentrated to dryness and the residue partitioned between EtOAc $(50 \mathrm{~mL})$ and water $(5 \mathrm{~mL})$. The organic phase was washed with water $(1 \times 5 \mathrm{~mL})$, dried over $\mathrm{MgSO}_{4}$ and concentrated to dryness. The residue was purified by mass-triggered preparative LCMS to afford (S)-4-hydroxy-3-(1-(3-isopropyl-5-(2-methyl-4(naphthalen-2-ylamino)benzamido)-2,6-dioxo-3,6-dihydropyrimidin-1(2H)-yl)cyclopropane-1-carbox-amido)butanoic acid (29, $53.0 \mathrm{mg}, 60 \%)$ as a cream coloured solid: $\mathrm{LCMS}\left(t_{\mathrm{R}}=1.15 \mathrm{~min}\right.$, purity $=98 \%$, MS ES ${ }^{-} \mathrm{m} / \mathrm{z} 612.55(\mathrm{M}-\mathrm{H})^{-} ;{ }^{1} \mathrm{H}$ NMR $(400 \mathrm{MHz}$, DMSO- $\left.d_{6}\right) \delta 8.83(\mathrm{~s}, 1 \mathrm{H}), 8.71(\mathrm{~s}, 1 \mathrm{H}), 8.40(\mathrm{~s}, 1 \mathrm{H}), 7.92-7.67(\mathrm{~m}, 3 \mathrm{H})$, $7.58(\mathrm{~d}, J=2.1 \mathrm{~Hz}, 1 \mathrm{H}), 7.50-7.59(\mathrm{~m}, 2 \mathrm{H}), 7.43$ (ddd, $J=8.2,6.8$, $1.3 \mathrm{~Hz}, 1 \mathrm{H}), 7.37-7.28(\mathrm{~m}, 2 \mathrm{H}), 7.10-7.01(\mathrm{~m}, 2 \mathrm{H}), 4.76(\mathrm{~h}, J=6.9 \mathrm{~Hz}$, $1 \mathrm{H}), 4.04(\mathrm{~m}, 1 \mathrm{H}), 3.30-3.40(\mathrm{~m}, 1 \mathrm{H}), 3.28-3.07(\mathrm{~m}, 1 \mathrm{H}), 2.44(\mathrm{~s}$, $3 \mathrm{H}), 2.38-2.21(\mathrm{~m}, 1 \mathrm{H}), 1.75-1.52(\mathrm{~m}, 2 \mathrm{H}), 1.32(\mathrm{~m}, 6 \mathrm{H}), 1.11(\mathrm{~s}, 2 \mathrm{H})$; ${ }^{13} \mathrm{C}$ NMR (101 MHz, DMSO- $\left.d_{6}\right) \delta 172.78,169.06,167.23,160.13$, 149.86, 145.39, 140.14, 134.26, 138.31, 129.48, 129.00, 128.73, 128.33, 127.51, 126.52, 126.41, 126.11, 123.47, 120.60, 118.23, 113.91, 112.85, 111.53, 62.51, 59.80, 48.79, 48.06, 37.48, 20.59, 18.82, 14.14; HRMS: $(\mathrm{M}+\mathrm{H})^{+}$calculated for $\mathrm{C}_{33} \mathrm{H}_{35} \mathrm{~N}_{5} \mathrm{O}_{7}$ 614.2536; found 614.2606; $[\alpha]_{\mathrm{D}}^{20}=-10.0^{\circ}(\mathrm{c}=2.5 \mathrm{~g} / \mathrm{L}, \mathrm{ACN})$; analytical chiral HPLC conditions (Chiralpak ID $4.6 \mathrm{~mm}^{*} 250 \mathrm{~mm} 5 \mu \mathrm{m}$ column; eluent, $n$-heptane/ $\mathrm{EtOH} / \mathrm{AcOH}$ (80/20/0.1); flow rate, $1.5 \mathrm{~mL} / \mathrm{min}$.; run time, $50 \mathrm{~min}$; $t_{\mathrm{R}}=23.959 \mathrm{~min}$ ( $S$ enantiomer $), t_{\mathrm{R}}=30.891 \mathrm{~min}$ ( $R$ enantiomer $)$, e.r. $=95: 5(S / R)$.

4.4.2. (S,E)-4-((benzyloxy)imino)-3-(1-(3-isopropyl-5-(2-methyl4-(naphthalen-2-ylamino)benzamido)-2,6-dioxo-3,6-dihydropyrimidin-1(2H)-yl)cyclopropane-1-carboxamido)butanoic acid (30)

To a stirred solution of $N$-\{3-[1-((S)-2-hydroxy-5-oxo-tetrahydro-furan-3-ylcarbamoyl)-cyclopropyl]-1-isopropyl-2,4-dioxo1,2,3,4-tetrahydro-pyrimidin-5-yl\}-2-methyl-4-(naphthalen-2ylamino)-benzamide (16, $103 \mathrm{mg}, 0.17 \mathrm{mmol}, 1.00 \mathrm{eq}$.) and pyridine $(27.2 \mu \mathrm{L}, 0.34 \mathrm{mmol}, 2.00$ eq. $)$ in a mixture of THF $(2.58 \mathrm{~mL})$ and $\mathrm{MeOH}(2.58 \mathrm{~mL})$, was added 0 -benzylhydroxylamine $(52.3 \mu \mathrm{L}$, $0.51 \mathrm{mmol}, 3.00$ eq.) and the reaction mixture was heated at $80^{\circ} \mathrm{C}$ for $1 \mathrm{~h}$. The resulting solution was cooled to r.t. and partitioned between EtOAc $(20 \mathrm{~mL})$ and a saturated aqueous solution of $\mathrm{NH}_{4} \mathrm{Cl}$ $(5 \mathrm{~mL})$. The organic phase was retained and the aqueous phase was washed with EtOAc $(3 \times 5 \mathrm{~mL})$. The organic phases were combined, dried over $\mathrm{MgSO}_{4}$ and concentrated to dryness and the residue was purified by flash chromatography (silica gel, gradient of $0-5 \%$ $\mathrm{MeOH}$ in DCM) to afford (S,E)-4-((benzyloxy)imino)-3-(1-(3isopropyl-5-(2-methyl-4-(naphthalen-2-ylamino)benzamido)-2,6- 
dioxo-3,6-dihydro-pyrimidin-1(2H)-yl)cyclopropane-1carboxamido)-butanoic acid (30, $103 \mathrm{mg}, 83 \%$ ) as a cream coloured solid: LCMS $\left(t_{\mathrm{R}}=1.30 \mathrm{~min}\right.$, purity $\left.=100 \%\right), \mathrm{ESI}^{-} \mathrm{m} / \mathrm{z} 715.28(\mathrm{M}-\mathrm{H})^{-}$; ${ }^{1} \mathrm{H}$ NMR (400 MHz, DMSO-d $\left.d_{6}\right){ }^{1} \mathrm{H}$ NMR ( $400 \mathrm{MHz}$, DMSO-d $\left.d_{6}\right) \delta 12.35$ (br s, $1 \mathrm{H}), 8.99-8.84(\mathrm{~m}, 1 \mathrm{H}), 8.76(\mathrm{~s}, 1 \mathrm{H}), 8.46(\mathrm{~d}, J=2.5 \mathrm{~Hz}, 1 \mathrm{H})$, $8.22(\mathrm{~s}, 1 \mathrm{H}), 8.04-7.75(\mathrm{~m}, 3 \mathrm{H}), 7.63(\mathrm{~d}, J=2.5 \mathrm{~Hz}, 1 \mathrm{H}), 7.59-7.43(\mathrm{~m}$, 2H), 7.42-7.29 (m, 5H), 7.20-6.94 (m, 2H), $6.77(\mathrm{~d}, J=5.8 \mathrm{~Hz}, 1 \mathrm{H})$, $5.13(\mathrm{~s}, 0.5 \mathrm{H}), 5.04(\mathrm{~s}, 1.5 \mathrm{H}), 4.93-4.67(\mathrm{~m}, 1 \mathrm{H}), 4.05(\mathrm{~m}, 1 \mathrm{H})$, 2.81-2.62 (m, 2H), 2.49 (s, 3H), $1.68(\mathrm{~m}, 2 \mathrm{H}), 1.37(\mathrm{~m}, 6 \mathrm{H}), 1.26-1.11$ $(\mathrm{m}, 2 \mathrm{H}) ;{ }^{13} \mathrm{C}$ NMR (DMSO- $\left.d_{6}\right) \delta 171.50,169.25,167.24,149.74,145.38$, $140.14,138.32,137.31,134.26,129.49,128.99,128.72,128.41,128.33$, 127.86, 127.61, 127.51, 126.51, 126.41, 126.12, 123.47, 118.23, 120.59, $113.96,112.84,111.52,75.24,47.99,46.01,45.76,40.13,37.36,20.99$, 20.77, 20.58; HRMS $(\mathrm{M}+\mathrm{H})^{+}$calculated for $\mathrm{C}_{40} \mathrm{H}_{40} \mathrm{~N}_{6} \mathrm{O}_{7}$ 717.29585; found $717.30273 ;[\alpha]_{\mathrm{D}}^{20}=-10.0^{\circ}(\mathrm{c}=2.5 \mathrm{~g} / \mathrm{L}, \mathrm{ACN})$; analytical chiral HPLC conditions (Chiralpak ID $4.6 \mathrm{~mm}^{*} 250 \mathrm{mml} 5 \mu \mathrm{m}$ column; eluent, $n$-heptane/EtOH/AcOH (80/20/0.1); flow rate, $1.5 \mathrm{~mL} / \mathrm{min}$; run time, $50 \mathrm{~min} ; t_{\mathrm{R}}=27.857 \mathrm{~min}$ ( $S$ enantiomer), $t_{\mathrm{R}}=35.022 \mathrm{~min}$ $(R$ enantiomer $)$, e.r. $=93: 7(S / R)$.

4.5. $N-(3-(1-(((3 S)-2-H y d r o x y-3-m e t h y l-5-o x o t e t r a h y d r o-f u r a n-3-$ yl)amino)-1-oxopropan-2-yl)-1-methyl-2,4-dioxo-1,2,3,4tetrahydropyrimidin-5-yl)-4-(quinoxalin-2-ylamino)benzamide (36) was prepared in 6 steps

4.5.1. (S)-2-((((9H-Fluoren-9-yl)methoxy)carbonyl)amino)-4-(tertbutoxy)-2-methyl-4-oxo-butanoic acid (32)

To a stirred solution of $10 \% \mathrm{w} / \mathrm{v} \mathrm{Na} \mathrm{CO}_{3}$ ( $4.17 \mathrm{~g}, 39.4 \mathrm{mmol}, 4.00$ eq.), was added dropwise a solution of (S)-2-amino-2-methyl-succinic acid 4-tert-butyl ester (31, $2.00 \mathrm{~g}, 9.84 \mathrm{mmol}, 1.00$ eq.) in 1,4dioxanne $(50 \mathrm{~mL})$. After $10 \mathrm{~min}$, a solution of Fmoc-OSu (3.98 g, $11.81 \mathrm{mmol}, 1.20$ eq.) in 1,4-dioxanne $(40 \mathrm{~mL})$ was added to the reaction mixture and the resulting suspension was stirred at r.t. overnight. The reaction mixture was diluted with water $(20 \mathrm{~mL})$ and the $\mathrm{pH}$ adjusted to 6 with $1 \mathrm{~N} \mathrm{HCl}(\mathrm{aq})$. The product was extracted with EtOAc $(3 \times 50 \mathrm{~mL})$ and the organic phases were combined and washed with water $(3 \times 20 \mathrm{~mL})$, dried over $\mathrm{MgSO}_{4}$ and concentrated to dryness to afford the crude product. The crude product was purified by flash chromatography (silica gel, gradient $0-10 \% \mathrm{MeOH}$ in DCM) to afford (S)-2-(9H-fluoren-9-ylmethoxycarbonylamino)-2methyl-succinic acid 4-tert-butyl ester (32, 3.54 g, 85\%) as a white solid: LCMS $\left(t_{\mathrm{R}}=1.22 \mathrm{~min}\right.$, purity $\left.=99 \%\right), \mathrm{MS} \mathrm{ES}^{-} \mathrm{m} / \mathrm{z} 424(\mathrm{M}-\mathrm{H})^{-}$; ${ }^{1} \mathrm{H} \mathrm{NMR}\left(400 \mathrm{MHz}\right.$, DMSO- $\left.d_{6}\right) \delta 12.8$ (br s, $\left.1 \mathrm{H}\right), 7.90(\mathrm{~d}, J=7.4 \mathrm{~Hz}, 1 \mathrm{H})$, $7.71(\mathrm{dd}, J=7.7,2.6 \mathrm{~Hz}, 1 \mathrm{H}), 7.42(\mathrm{td}, J=7.5,1.1 \mathrm{~Hz}, 1 \mathrm{H}), 7.33(\mathrm{t}$, $J=7.5 \mathrm{~Hz}, 1 \mathrm{H}), 4.34-4.13(\mathrm{~m}, 3 \mathrm{H}), 2.87(\mathrm{~d}, J=14.3 \mathrm{~Hz}, 1 \mathrm{H}), 2.76-2.60$ (m, 1H), $1.43(\mathrm{~s}, 3 \mathrm{H}), 1.35(\mathrm{~s}, 9 \mathrm{H})$.

4.5.2. tert-Butyl (S)-3-((((9H-fluoren-9-yl)methoxy)carbonyl)amino)-4-hydroxy-3-methylbutanoate

To a stirred solution of (S)-2-(9H-fluoren-9-ylmethoxycarbonylamino)-2-methyl-succinic acid 4-tert-butyl ester (32, $2.00 \mathrm{~g}, 4.72 \mathrm{mmol}, 1.00$ eq.), $N$-methylmorpholine $(1.03 \mathrm{~mL}$, $9.44 \mathrm{mmol}, 2.00$ eq.) in $\mathrm{DCM}(40 \mathrm{~mL})$ at $0^{\circ} \mathrm{C}$, was added isobutyl chloroformate $(1.22 \mathrm{~mL}, 9.44 \mathrm{~mol}, 2.00$ eq.) and the solution was stirred at $0{ }^{\circ} \mathrm{C}$ for $1 \mathrm{~h}$. Solid sodium borohydride (534 mg, $14.2 \mathrm{~mol}$, 3.00 eq.) was added and the reaction mixture was stirred at $0{ }^{\circ} \mathrm{C}$ for $1 \mathrm{~h}$ and at r.t. overnight. The reaction mixture was washed with water $(3 \times 5 \mathrm{~mL})$, dried over $\mathrm{MgSO}_{4}$ and concentrated to dryness to afford $(S)-2-((((9 H$-fluoren-9-yl)methoxy)carbonyl)amino)-4-(tertbutoxy)-2-methyl-4-oxo-butanoic acid $(1.90 \mathrm{~g}, 98 \%)$ as a white solid: LCMS ( $t_{\mathrm{R}}=1.25 \mathrm{~min}$, purity $\left.=99 \%\right), \quad \mathrm{MS} \quad \mathrm{ESI}^{+} \mathrm{m} / \mathrm{z} 434$ $(\mathrm{M}+\mathrm{Na}){ }^{+} ;{ }^{1} \mathrm{H}$ NMR $\left(400 \mathrm{MHz}\right.$, DMSO- $\left.d_{6}\right) \delta 7.95-7.84(\mathrm{~m}, 1 \mathrm{H}), 7.73$ $(\mathrm{d}, J=7.5 \mathrm{~Hz}, 1 \mathrm{H}), 7.42(\mathrm{td}, J=7.5,1.2 \mathrm{~Hz}, 1 \mathrm{H}), 7.33(\mathrm{tt}, J=7.5,1.1 \mathrm{~Hz}$, $1 \mathrm{H}), 6.84(\mathrm{br} \mathrm{s}, 1 \mathrm{H}), 4.79(\mathrm{~s}, 1 \mathrm{H}), 4.21(\mathrm{~d}, J=4.6 \mathrm{~Hz}, 2 \mathrm{H}), 3.76(\mathrm{~m}, 2 \mathrm{H})$, 2.90-2.68 (m, 1H), $2.51(\mathrm{~m}, 1 \mathrm{H}), 1.36(\mathrm{~s}, 9 \mathrm{H}), 1.23(\mathrm{~s}, 3 \mathrm{H})$.
4.5.3. tert-Butyl (S)-3-amino-4-hydroxy-3-methylbutanoate (33)

To a stirred solution of (S)-3-(9H-fluoren-9-ylmethoxycarbonylamino)-4-hydroxy-3-methyl-butyric acid tert-butyl ester $(1.50 \mathrm{~g}, 3.65 \mathrm{mmol}, 1.00 \mathrm{eq}$.) in $\mathrm{MeCN}(15.0 \mathrm{~mL})$ at r.t., was added pyrrolidine ( $60.2 \mu \mathrm{L}, 0.73 \mathrm{mmol}, 0.20 \mathrm{eq}$.). The reaction mixture was stirred at r.t. for $24 \mathrm{~h}$ and concentrated to dryness. The residue was suspended in toluene and concentrated to dryness to afford tertbutyl (S)-3-amino-4-hydroxy-3-methylbutanoate (690 mg, quant.) as a pale yellow gum that was used without further purification: LCMS $\left(t_{\mathrm{R}}=1.25 \mathrm{~min}\right.$, purity $\left.=99 \%\right), \mathrm{ESI}^{+} \mathrm{m} / z 134\left(\mathrm{M}-\mathrm{C}_{4} \mathrm{H}_{9}+\mathrm{H}\right)^{+}$.

4.5.4. tert-Butyl (3S)-4-hydroxy-3-methyl-3-(2-(3-methyl-2,6dioxo-5-(4-(quinoxalin-2-ylamino)benzamido)-3,6-dihydropyrimidin-1(2H)-yl)propanamido)butanoate (35)

To a stirred solution of 2-\{3-methyl-2,6-dioxo-5-[4-(quinoxalin2-ylamino)-benzoylamino]-3,6-dihydro-2H-pyrimidin-1-yl\}-propionic acid $(34,120 \mathrm{mg}, 0.26 \mathrm{mmol}, 1.00$ eq.) and $N$-methylmorpholine ( $34.4 \mu \mathrm{L}, 0.31 \mathrm{mmol}, 1.20$ eq.) in DMF ( $2.40 \mathrm{~mL})$ at r.t., was added HATU $(119 \mathrm{mg}, 0.31 \mathrm{mmol}, 1.20$ eq.) and the reaction mixture was stirred for $1 \mathrm{~h} 30$ at r.t. then tert-butyl (S)-3-amino-4hydroxy-3-methylbutanoate (33, $98.7 \mathrm{mg}, 0.52 \mathrm{mmol}, 2.00 \mathrm{eq}$.) was added and the reaction mixture was heated at $80^{\circ} \mathrm{C}$ for $4 \mathrm{~h}$ and left to stir at r.t. for $48 \mathrm{~h}$. The reaction mixture was partitioned between EtOAc $(50 \mathrm{~mL})$ and water $(5 \mathrm{~mL})$. The organic phase was retained and the aqueous phases washed with EtOAC $(3 \times 10 \mathrm{~mL})$. The organic phases were combined, washed with water $(4 \times 10 \mathrm{~mL})$, dried over $\mathrm{MgSO}_{4}$ and concentrated to dryness. The residue was purified by flash chromatography (silica gel, gradient of $0-100 \%$ EtOAc in heptane) to afford tert-butyl (3S)-4-hydroxy-3-methyl-3(2-(3-methyl-2,6-dioxo-5-(4-(quinoxalin-2-ylamino)benzamido)3,6-dihydropyrimidin-1(2H)-yl)propanamido)butanoate $100 \mathrm{mg}, 45 \%)$ as a yellow solid as a mixture of diastereoisomers: LCMS $\left(t_{\mathrm{R}}=1.19 \mathrm{~min}\right.$, purity $\left.=74 \%\right), \mathrm{ESI}^{+} \mathrm{m} / z 576.2\left(\mathrm{M}-\mathrm{C}_{4} \mathrm{H}_{9}+\mathrm{H}\right)^{+}$, $632.4(\mathrm{M}+\mathrm{H})^{+} ;{ }^{1} \mathrm{H}$ NMR $\left(400 \mathrm{MHz}\right.$, DMSO- $\left.d_{6}\right) \delta 10.29(\mathrm{~s}, 1 \mathrm{H}), 9.15(\mathrm{~d}$, $J=6.3 \mathrm{~Hz}, 1 \mathrm{H}), 8.63(\mathrm{~s}, 1 \mathrm{H}), 8.35(\mathrm{~d}, J=10.3 \mathrm{~Hz}, 1 \mathrm{H}), 8.13$ (d, $J=8.7 \mathrm{~Hz}, 2 \mathrm{H}), 8.02-7.94(\mathrm{~m}, 3 \mathrm{H}), 7.91(\mathrm{dd}, J=8.1,1.5 \mathrm{~Hz}, 1 \mathrm{H}), 7.82$ $(\mathrm{dd}, J=8.4,1.3 \mathrm{~Hz}, 1 \mathrm{H}), 7.71$ (ddd, $J=8.4,6.9,1.5 \mathrm{~Hz}, 1 \mathrm{H}), 7.54(\mathrm{ddd}$, $J=8.3,6.9,1.4 \mathrm{~Hz}, 1 \mathrm{H}), 6.99(\mathrm{~d}, J=21.4 \mathrm{~Hz}, 1 \mathrm{H}), 5.28(\mathrm{~m}, 1 \mathrm{H}), 4.72(\mathrm{t}$, $J=6.1 \mathrm{~Hz}, 0.5 \mathrm{H}), 4.59(\mathrm{t}, J=6.1 \mathrm{~Hz}, 0.5 \mathrm{H}), 3.52(\mathrm{~m}, 2 \mathrm{H}), 3.42-3.34$ $(\mathrm{d}, J=1.9 \mathrm{~Hz}, 3 \mathrm{H}), 2.67-2.55(\mathrm{~m}, 1 \mathrm{H}), 2.46-2.23(\mathrm{~m}, 1 \mathrm{H}), 1.46$ (overlapping $\mathrm{d}, 3 \mathrm{H}), 1.40(\mathrm{~s}, 9 \mathrm{H}), 1.26(\mathrm{~s}, 3 \mathrm{H})$.

4.5.5. tert-Butyl (3S)-4-hydroxy-3-methyl-3-(2-(3-methyl-2,6dioxo-5-(4-(quinoxalin-2-ylamino)benzamido)-3,6-dihydropyrimidin-1(2H)-yl)propanamido)butanoate

To a stirred solution of tert-butyl (3S)-4-hydroxy-3-methyl-3(2-(3-methyl-2,6-dioxo-5-(4-(quinoxalin-2-ylamino)benzamido)3,6-dihydropyrimidin-1(2H)-yl)propanamido)butanoate (35, $100 \mathrm{mg}, 0.11 \mathrm{mmol}, 1.00$ eq. $)$ in $\mathrm{DCM}(1.40 \mathrm{~mL})$ at $0^{\circ} \mathrm{C}$, was added Dess-Martin periodinane (1,1,1-tris(acetyloxy)-1,1-dihydro-1,2benziodoxol-3-(1H)-one) $(56.4 \mathrm{mg}, 0.13 \mathrm{mmol}, 1.20$ eq.) and the reaction mixture was stirred overnight at r.t. The reaction mixture was diluted with DCM $(20 \mathrm{~mL})$, washed with $10 \% \mathrm{w} / \mathrm{v} \mathrm{NaHSO}_{4}(\mathrm{aq})$, dried over $\mathrm{MgSO}_{4}$ and concentrated to dryness to afford tert-butyl (3S)-4-hydroxy-3-methyl-3-(2-(3-methyl-2,6-dioxo-5-(4-(quinoxalin-2-ylamino)benzamido)-3,6-dihydropyrimidin-1(2H)-yl)propanamido)butanoate (100 $\mathrm{mg}$, quant.) as a complex mixture of diastereisomers: LCMS $\left(t_{\mathrm{R}}=1.22 \mathrm{~min}\right.$, purity $\left.=96 \%\right), \mathrm{MS} \mathrm{ESI}^{+} \mathrm{m} / z$ $630.4(\mathrm{M}+\mathrm{H})^{+} ;{ }^{1} \mathrm{H}$ NMR $\left(400 \mathrm{MHz}\right.$, DMSO-d $\left.d_{6}\right) \delta 10.30(\mathrm{~s}, 1 \mathrm{H}), 9.28$ (d, $J=16.0 \mathrm{~Hz}, 1 \mathrm{H}), 9.13(\mathrm{~d}, J=27.6 \mathrm{~Hz}, 1 \mathrm{H}), 8.63(\mathrm{~s}, 1 \mathrm{H}), 8.36$ (d, $J=3.9 \mathrm{~Hz}, 1 \mathrm{H}), 8.26(\mathrm{~d}, J=5.5 \mathrm{~Hz}, 1 \mathrm{H}), 8.18-7.95(\mathrm{~m}, 3 \mathrm{H}), 7.94-7.85$ $(\mathrm{m}, 4 \mathrm{H}), 7.85-7.76(\mathrm{~m}, 4 \mathrm{H}), 7.71$ (ddd, $J=8.4,7.0,1.6 \mathrm{~Hz}, 2 \mathrm{H})$, 7.60-7.47 (m, 2H), $5.31(\mathrm{~m}, 1 \mathrm{H}), 3.40(\mathrm{~d}, J=3.5 \mathrm{~Hz}, 3 \mathrm{H}), 2.81-2.59$ $(\mathrm{m}, 2 \mathrm{H}), 1.47$ (overlapping d, 3H), 1.40 (overlapping s, 9H), 1.28-1.22 (overlapping s, $3 \mathrm{H}$ ). 
4.5.6. $N$-(3-(1-(((3S)-2-Hydroxy-3-methyl-5-oxotetrahydro-furan3-yl)amino)-1-oxopropan-2-yl)-1-methyl-2,4-dioxo-1,2,3,4tetrahydropyrimidin-5-yl)-4-(quinoxalin-2-ylamino)benzamide (36)

To a stirred solution of tert-butyl (3S)-4-hydroxy-3-methyl-3(2-(3-methyl-2,6-dioxo-5-(4-(quinoxalin-2-ylamino)benzamido)3,6-dihydropyrimidin-1(2H)-yl)propanamido)butanoate (100 mg, $0.11 \mathrm{mmol}, 1.00$ eq. $)$ in DCM $(700 \mu \mathrm{L})$ at r.t., was added TFA $(60 \mu \mathrm{L}$, $0.78 \mathrm{mmol}, 7.00$ eq.) and the reaction mixture is stirred at r.t. for $1 \mathrm{~h}$. The reaction mixture was concentrated and the residue was purified by flash chromatography (silica gel, gradient of $0-5 \%$ $\mathrm{MeOH}$ in DCM) to afford $N$-(3-(1-(((3S)-2-Hydroxy-3-methyl-5oxotetrahydrofuran-3-yl)amino)-1-oxopropan-2-yl)-1-methyl2,4-dioxo-1,2,3,4-tetrahydropyrimidin-5-yl)-4-(quinoxalin-2ylamino)benzamide $(\mathbf{3 5}, 25.0 \mathrm{mg}, 36 \%)$ as a beige solid: LCMS $\left(t_{\mathrm{R}}=0.97 \mathrm{~min}\right.$, purity $\left.=96 \%\right), \mathrm{ESI}^{+} \mathrm{m} / z 574.23(\mathrm{M}+\mathrm{H})^{+} ;{ }^{1} \mathrm{H} \mathrm{NMR}$ $\left(400 \mathrm{MHz}, \mathrm{DMSO}-d_{6}\right) \delta 10.36(\mathrm{~s}, 1 \mathrm{H}), 9.16(\mathrm{~s}, 1 \mathrm{H}), 8.64(\mathrm{~s}, 1 \mathrm{H}), 8.34$ (d, $J=9.4 \mathrm{~Hz}, 1 \mathrm{H}), 8.14(\mathrm{~d}, J=8.6 \mathrm{~Hz}, 2 \mathrm{H}), 7.99(\mathrm{~d}, J=8.6 \mathrm{~Hz}, 2 \mathrm{H})$, $7.92(\mathrm{~d}, J=8.0 \mathrm{~Hz}, 1 \mathrm{H}), 7.82(\mathrm{~d}, J=8.0 \mathrm{~Hz}, 1 \mathrm{H}), 7.71(\mathrm{t}, J=7.7 \mathrm{~Hz}, 1 \mathrm{H})$, $7.54(\mathrm{t}, J=7.7 \mathrm{~Hz}, 1 \mathrm{H}), 5.28(\mathrm{br} \mathrm{m}, 1 \mathrm{H}), 3.38(\mathrm{~d}, J=3.7 \mathrm{~Hz}, 3 \mathrm{H})$, 2.52-2.49 (hidden m, 2H), 1.45 (br s, 3H), 1.31 (br s, 3H).

4.6. $N-(3-(1-(((3 R)-2-h y d r o x y-5-o x o t e t r a h y d r o f u r a n-3-y l)-$ carbamoyl)cyclopropyl)-1-isopropyl-2,4-dioxo-1,2,3,4tetrahydropyrimidin-5-yl)-2-methyl-4-(naphthalen-2-ylamino) benzamide (28) was prepared in 6 steps

\subsection{1. (R)-2-(((Allyloxy)carbonyl)amino)-4-(tert-butoxy)-4-oxo-} butanoic acid

To a cooled $\left(4^{\circ} \mathrm{C}\right)$ solution of D-aspartic acid 4-tert-butyl ester ( $15.0 \mathrm{~g}, 78.3 \mathrm{mmol})$ in $\mathrm{THF} / \mathrm{H}_{2} \mathrm{O}(50 \mathrm{~mL} / 150 \mathrm{~mL}$ ) were added sodium bicarbonate $(26.6 \mathrm{~g}, 317.1 \mathrm{mmol})$ and allyl chloroformate $(15.17 \mathrm{~mL}$, $57 \mathrm{~g}, 142.7 \mathrm{mmol}$ ). After $3 \mathrm{~h}$ of stirring at room temperature, the medium was extracted with EtOAc (three times). The aqueous layer was acidified with aqueous $\mathrm{HCl}(6 \mathrm{~N})$ until obtaining $\mathrm{pH} 2$. The aqueous layer was extracted (EtOAc, three times). The combined organic layer was dried over sodium sulphate, filtered and concentrated in vacuo to afford the title compound $(18.7 \mathrm{~g}, 87 \%$, as colorless oil): LCMS ESI ${ }^{-} \mathrm{m} / z 272.4(\mathrm{M}-\mathrm{H})^{-} ;{ }^{1} \mathrm{H}$ NMR $\left(\mathrm{CDCl}_{3}\right.$, $500 \mathrm{MHz}) \delta 5.96-5.87(\mathrm{~m}, 1 \mathrm{H}), 5.74(\mathrm{br} \mathrm{d}, J=8.5 \mathrm{~Hz}, 1 \mathrm{H}), 5.31(\mathrm{~m}$, $1 \mathrm{H}), 5.22(\mathrm{~m}, 1 \mathrm{H}), 4.65-4.55(\mathrm{~m}, 3 \mathrm{H}), 2.97(\mathrm{dd}, J=17.0 \mathrm{~Hz}$ and $4.0 \mathrm{~Hz}, 1 \mathrm{H}), 2.76(\mathrm{dd}, J=17.0 \mathrm{~Hz}$ and $5.0 \mathrm{~Hz}, 1 \mathrm{H}), 1.44(\mathrm{~s}, 9 \mathrm{H})$.

\subsection{2. tert-Butyl (R)-3-(((allyloxy)carbonyl)amino)-4-}

(methoxy(methyl)amino)-4-oxo-butanoate

To a cooled solution of (R)-2-(((allyloxy)carbonyl)amino)-4(tert-butoxy)-4-oxobutanoic acid $(18.7 \mathrm{~g}, 68.7 \mathrm{mmol})$ in DCM $(390 \mathrm{~mL})$ were added $\mathrm{N}, \mathrm{O}$-dimethylhydroxylamine hydrochloride $(8.00 \mathrm{~g}, 82.4 \mathrm{mmol}), 4$-methylmorpholine $(9.07 \mathrm{~mL}, 82.4 \mathrm{mmol})$ and 1,(3-dimethylaminopropyl)-3-ethylcarbodiimide hydro-chloride $(15.8 \mathrm{~g}, 82.4 \mathrm{mmol})$. After $3 \mathrm{~h}$ of stirring at room temperature, the medium was washed with aqueous $\mathrm{HCl}$ ( $1 \mathrm{~N}$, two times), with brine, dried over sodium sulphate, filtered and concentrated in vacuo. Purification of the residue by chromatography on silica gel (cyclohexane-EtOAc, gradient of $9 / 1$ to $7 / 3$ ) afforded the title compound (15.3 g, 71\%, as yellow oil): LCMS ESI ${ }^{+} \mathrm{m} / \mathrm{z} 317.3(\mathrm{M}+\mathrm{H})^{+} ;{ }^{1} \mathrm{H}$ NMR $\left(\mathrm{CDCl}_{3}, 500 \mathrm{MHz}\right) \delta 5.93-5.85(\mathrm{~m}, 1 \mathrm{H}), 5.59(\mathrm{br} \mathrm{d}, J=8.5 \mathrm{~Hz}, 1 \mathrm{H})$, $5.30(\mathrm{~m}, 1 \mathrm{H}), 5.19(\mathrm{~m}, 1 \mathrm{H}), 5.00(\mathrm{~m}, 1 \mathrm{H}), 4.56(\mathrm{~m}, 2 \mathrm{H}), 3.78(\mathrm{~s}, 3 \mathrm{H})$, $3.21(\mathrm{~s}, 3 \mathrm{H}), 2.70(\mathrm{dd}, J=15.0 \mathrm{~Hz}$ and $5.5 \mathrm{~Hz}, 1 \mathrm{H}), 2.54(\mathrm{dd}$, $J=15.0 \mathrm{~Hz}$ and $6.5 \mathrm{~Hz}, 1 \mathrm{H}), 1.43(\mathrm{~s}, 9 \mathrm{H})$.

4.6.3. tert-Butyl (R)-3-(((allyloxy)carbonyl)amino)-4-oxobutanoate

A solution of lithium aluminium hydride $(2 \mathrm{~N}$ in THF, $8.22 \mathrm{~mL}$, $16.4 \mathrm{mmol})$ was added dropwise to a cooled $\left(-78^{\circ} \mathrm{C}\right)$ solution of tert-butyl (R)-3-(((allyloxy)carbonyl)amino)-4-(methoxy-(methyl) amino)-4-oxobutanoate $(8.0 \mathrm{~g}, 25.3 \mathrm{mmol})$ in anhydrous THF $(107 \mathrm{~mL})$. The mixture was stirred at $-78^{\circ} \mathrm{C}$ for $3 \mathrm{~h}$, then aqueous $\mathrm{HCl}(1 \mathrm{~N})$ was slowly added to the medium, and the temperature was allowed to warm to $0^{\circ} \mathrm{C}$. The mixture was diluted with EtOAc. The aqueous layer was extracted (EtOAc). The combined organic layer was washed with aqueous $\mathrm{HCl}(1 \mathrm{~N})$, with brine, dried over sodium sulphate, filtered and concentrated in vacuo to afford the title compound (6.65 g, quantitative, as pale yellow oil): ${ }^{1} \mathrm{H}$ NMR $\left(\mathrm{CDCl}_{3}, 500 \mathrm{MHz}\right) \delta 9.66(\mathrm{~s}, 1 \mathrm{H}), 5.83(\mathrm{br} \mathrm{d}, 1 \mathrm{H}), 5.99-5.82(\mathrm{~m}, 1 \mathrm{H})$, $5.33(\mathrm{~m}, 1 \mathrm{H}), 5.24(\mathrm{~m}, 1 \mathrm{H}), 4.61(\mathrm{~m}, 2 \mathrm{H}), 4.36(\mathrm{~m}, 1 \mathrm{H}), 2.96(\mathrm{dd}$, $J=17.2 \mathrm{~Hz}$ and $4.5 \mathrm{~Hz}, 1 \mathrm{H}), 2.76(\mathrm{dd}, J=17.2 \mathrm{~Hz}$ and $5.1 \mathrm{~Hz}, 1 \mathrm{H}), 1.43$ (s, 9H).

\subsection{4. tert-Butyl (R)-3-(((allyloxy)carbonyl)amino)-4,4-diethoxy-} butanoate

To a freshly prepared solution of tert-butyl (R)-3-(((allyloxy) carbonyl)amino)-4-oxobutanoate $(6.65 \mathrm{~g}, 25.8 \mathrm{mmol})$ in absolute ethanol $(28 \mathrm{~mL})$ were added, under Argon atmosphere, triethyl orthoformate (12.9 mL, $77.5 \mathrm{mmol})$, $p$-toluenesulfonic acid (133 mg, $0.77 \mathrm{mmol}$ ) and $3 \AA$ molecular sieves. After stirring at room temperature for 3 days, the mixture was filtered over a pad of celite and rinsed with EtOH. The filtrate was concentrated under reduced pressure. Purification of the residue by flash chromatography on silica gel (cyclohexane-EtOAc, 9/1) afforded the title compound $(4.87 \mathrm{~g}, 50 \%)$ as colorless oil): ${ }^{1} \mathrm{H}$ NMR $\left(\mathrm{CDCl}_{3}, 400 \mathrm{MHz}\right)$ $\delta 5.83-6.00(\mathrm{~m}, 1 \mathrm{H}), 5.30(\mathrm{~d}, J=17.4 \mathrm{~Hz}, 1 \mathrm{H}), 5.23(\mathrm{br} \mathrm{d}, J=8.5 \mathrm{~Hz}$, $1 \mathrm{H}), 5.21(\mathrm{~m}, 1 \mathrm{H}), 4.56(\mathrm{~m}, 2 \mathrm{H}), 4.49(\mathrm{~m}, 1 \mathrm{H}), 4.22-4.11(\mathrm{~m}, 1 \mathrm{H})$, $3.65-3.78(\mathrm{~m}, 2 \mathrm{H}), 3.48-3.61(\mathrm{~m}, 2 \mathrm{H}), 2.56(\mathrm{dd}, J=15.6 \mathrm{~Hz}$ and $5.7 \mathrm{~Hz}, 1 \mathrm{H}), 2.45(\mathrm{dd}, J=15.6 \mathrm{~Hz}$ and $7.2 \mathrm{~Hz}, 1 \mathrm{H}), 1.44(\mathrm{~s}, 9 \mathrm{H}), 1.21(\mathrm{~m}$, $6 \mathrm{H}) ;{ }^{13} \mathrm{C}$ NMR (101 MHz, DMSO-d $\left.d_{6}\right) \delta 170.12,155.44,133.78,116.66$, 102.57, 79.66, 64.16, 63.39, 62.22, 50.44, 36.09, 27.69, 15.15; $[\alpha]_{\mathrm{D}}^{20}=+17.8^{\circ}(\mathrm{c}=18 \mathrm{~g} / \mathrm{L}, \mathrm{MeCN})$.

4.6.5. N-(3-(1-(((3R)-2-ethoxy-5-oxotetrahydrofuran-3-yl) carbamoyl)cyclopropyl)-1-isopropyl-2,4-dioxo-1,2,3,4tetrahydropyrimidin-5-yl)-2-methyl-4-(naphthalen-2-ylamino) benzamide

First Step: To a stirred solution of 1-\{3-isopropyl-5-[2-methyl-4(naphthalen-2-ylamino)-benzoylamino]-2,6-dioxo-3,6-dihydro$2 \mathrm{H}$-pyrimidin-1-yl\}-cyclopropanecarboxylic acid, $(283 \mathrm{mg}$, $0.55 \mathrm{mmol}, 1.00 \mathrm{eq}$.) and $N$-methyl morpholine $(72.9 \mu \mathrm{L}, 0.66 \mathrm{mmol}$, 1.20 eq.) in a mixture of DCM $(1.27 \mathrm{~mL})$ and DMF $(1.27 \mathrm{~mL})$, HATU (252 $\mathrm{mg}, 0.66 \mathrm{mmol}, 1.20 \mathrm{eq}$.) was added and the reaction mixture was stirred at r.t. for 45 min.

Second Step: In parallel, a solution of $((R)$-2-ethoxy-5-oxo-tetrahydro-furan-3-yl)-carbamic acid allyl ester, (190 mg, $0.83 \mathrm{mmol}, 1.40$ eq.) and 1,3-dimethylbarbituric acid (129 mg, $0.83 \mathrm{mmol}, 1.50$ eq.) in a mixture of DCM $(0.89 \mathrm{~mL})$ and $\mathrm{DMF}(0.63 \mathrm{~mL})$ at r.t. was degassed and purged with nitrogen then tetrakis(triphenylphosphine)palla$\operatorname{dium}(0)(25.5 \mathrm{mg}, 0.02 \mathrm{mmol}, 0.04$ eq.) was added. The reaction mixture was stirred at room temperature for $1 \mathrm{~h}$.

Third step: The product, (4S)-4-amino-5-ethoxydihydrofuran$2(3 H)$-one, from the Alloc deprotection step was added to the preactivated ester for the first step and the reaction mixture was stirred at room temperature overnight. The reaction mixture was treated with a saturated solution of $\mathrm{NaHCO}_{3}(\mathrm{aq})$ and the product extracted with EtOAc $(2 \times 20 \mathrm{~mL})$. The organic phases were combined and washed with water $(3 \times 5 \mathrm{~mL})$, dried over $\mathrm{MgSO}_{4}$ and concentrated to dryness. The crude product was purified by flash chromatography (silica gel, gradient of $0-5 \% \mathrm{MeOH}$ in DCM) to afford $N-\{3-[1-((R)-2-$ ethoxy-5-oxo-tetrahydro-furan-3-ylcarbamoyl)-cyclopropyl]-1isopropyl-2,4-dioxo-1,2,3,4-tetrahydro-pyrimidin-5-yl\}-2-methyl4-(naphthalen-2-ylamino)-benzamide (1.80 g, quant.) as an orange solid (mixture of diastereoisomers): LCMS $\left(t_{\mathrm{R}}=1.27 \mathrm{~min}\right.$, 
purity $=100 \%), \mathrm{ESI}^{+} \mathrm{m} / \mathrm{z} 640.20(\mathrm{M}+\mathrm{H})^{+} ;{ }^{1} \mathrm{H}$ NMR $(400 \mathrm{MHz}$, DMSO- $\left.d_{6}\right) \delta 8.96-8.75$ (overlapping $\left.\mathrm{s}, 1 \mathrm{H}\right), 8.70(\mathrm{~s}, 1 \mathrm{H}), 8.43(\mathrm{~s}, 1 \mathrm{H})$, $8.23(\mathrm{~d}, J=7.1 \mathrm{~Hz}, 0.5 \mathrm{H}), 8.05-7.91(\mathrm{~m}, 0.5 \mathrm{H}), 7.91-7.69(\mathrm{~m}, 3 \mathrm{H})$ 7.67-7.53 (m, 3H), 7.52-7.39 (m, 2H), 7.37-7.27 (m, 2H), 7.12-7.00 $(\mathrm{m}, 2 \mathrm{H}), 5.50(\mathrm{~d}, J=5.3 \mathrm{~Hz}, 0.5 \mathrm{H}), 5.22(\mathrm{~d}, J=6.0 \mathrm{~Hz}, 0.5 \mathrm{H}), 4.90-4.51$ $(\mathrm{m}, 1 \mathrm{H}), 4.04$ (overlapping signals, $0.5 \mathrm{H}), 3.84-3.47$ (overlapping signals, $2 \mathrm{H}), 2.99(\mathrm{~m}, 0.5 \mathrm{H}), 2.68-2.53(\mathrm{~m}, 1 \mathrm{H}), 2.44(\mathrm{~s}, 3 \mathrm{H}), 2.39-2.28$ $(\mathrm{m}, 1 \mathrm{H}), 1.83-1.53(\mathrm{~m}, 2 \mathrm{H}), 1.48-1.24(\mathrm{~m}, 6 \mathrm{H}), 1.23-1.06(\mathrm{~m}, 5 \mathrm{H})$.

\subsection{6. $\mathrm{N}-(3-(1-(((3 R)-2-H y d r o x y-5-o x o t e t r a h y d r o f u r a n-3-y l)$}

carbamoyl)cyclopropyl)-1-isopropyl-2,4-dioxo-1,2,3,4tetrahydropyrimidin-5-yl)-2-methyl-4-(naphthalen-2-ylamino) benzamide (28)

To a stirred solution of $\mathrm{N}$-\{3-[1-((S)-2-ethoxy-5-oxo-tetrahydrofuran-3-ylcarbamoyl)-cyclopropyl]-1-isopropyl-2,4-dioxo-1,2,3,4tetrahydro-pyrimidin-5-yl\}-2-methyl-4-(naphthalen-2-ylamino)benzamide $(280 \mathrm{mg}, 0.44 \mathrm{mmol}, 1.00$ eq.) in $\mathrm{MeCN}(5.60 \mathrm{~mL})$ was added $\mathrm{HCl}(\mathrm{aq})(2 \mathrm{M}, 2.19 \mathrm{~mL}, 4.38 \mathrm{mmol}, 10.0$ eq.) and the reaction mixture was stirred at room temperature for $5 \mathrm{~h}$. The volatiles were eliminated under a flow of nitrogen and the residue was treated with a saturated aqueous solution of $\mathrm{NaHCO}_{3}(\mathrm{pH} 8)$ then diluted with EtOAc. The organic phase was discarded and the $\mathrm{pH}$ of the aqueous phase was adjusted to 5 with $\mathrm{AcOH}$. The product was extracted in EtOAc $(3 \times 5 \mathrm{~mL})$, the organic phases were combined, dried over $\mathrm{MgSO} 4$ and concentrated to dryness. The resulting beige solid was crystallised from a mixture of EtOAc/toluene and the solid was recuperated by filtration and dried to a constant weight un a vacuum oven at $40^{\circ} \mathrm{C}$ to afford $N$-(3-(1-(((3R)-2-hydroxy-5oxotetrahydrofuran-3-yl)carbamoyl)cyclopropyl)-1-isopropyl-2,4dioxo-1,2,3,4-tetrahydro-pyrimidin-5-yl)-2-methyl-4-(naphthalen-2-ylamino)benzamide $(1.07 \mathrm{~g}, 65 \%)$ as an off-white solid: LCMS $\left(t_{\mathrm{R}}=1.19 \mathrm{~min}\right.$, purity $\left.=99 \%\right), \mathrm{ESI}^{+} \mathrm{m} / z 612.36(\mathrm{M}+\mathrm{H})^{+} ;{ }^{1} \mathrm{H}$ NMR $\left(400 \mathrm{MHz}\right.$, DMSO- $\left.d_{6}\right) \delta 8.96-8.83(\mathrm{~m}, 1 \mathrm{H}), 8.71(\mathrm{~s}, 1 \mathrm{H}), 8.42(\mathrm{~s}$, $1 \mathrm{H}), 8.21(\mathrm{br} \mathrm{s}, 1 \mathrm{H}), 7.87-7.73(\mathrm{~m}, 3 \mathrm{H}), 7.58(\mathrm{~d}, J=2.3 \mathrm{~Hz}, 1 \mathrm{H}), 7.50$ $(\mathrm{d}, J=8.4 \mathrm{~Hz}, 1 \mathrm{H}), 7.43(\mathrm{ddd}, J=8.2,6.8,1.3 \mathrm{~Hz}, 1 \mathrm{H}), 7.36-7.27(\mathrm{~m}$, $2 \mathrm{H}$ ), 7.07 (dd, $J=8.3,2.3 \mathrm{~Hz}, 1 \mathrm{H}), 7.03(\mathrm{~d}, J=2.3 \mathrm{~Hz}, 1 \mathrm{H}), 5.41$ (br s, $1 \mathrm{H}), 4.78(\mathrm{~m}, 1 \mathrm{H}), 4.15(\mathrm{~s} \mathrm{br}, 1 \mathrm{H}), 2.93(\mathrm{~m}, 1 \mathrm{H}), 2.44(\mathrm{~s}, 3 \mathrm{H}), 2.32(\mathrm{~m}$, 1H), 1.65 (m, 2H), 1.32 (dd, $J=13.1,6.7 \mathrm{~Hz}, 6 \mathrm{H}), 1.16(\mathrm{~m}, 2 \mathrm{H})$.

4.7. $\mathrm{N}-(3-(1-(((4 R)-2-h y d r o x y-6-o x o t e t r a h y d r o-2 H-p y r a n-4-y l)$ carbamoyl)cyclopropyl)-1-isopropyl-2,4-dioxo-1,2,3,4tetrahydropyrimidin-5-yl)-2-methyl-4-(naphthalen-2-ylamino)benzamide (37) was prepared in 7 steps

\subsection{1. tert-Butyl (R)-3-((((9H-fluoren-9-yl)methoxy)-carbonyl)-} amino)-5-(methoxy (methyl)amino)-5-oxopentanoate

To a cooled solution of Fmoc- $\beta$-homoaspartic acid(O- $t \mathrm{Bu})(20.0 \mathrm{~g}$, $47.0 \mathrm{mmol})$ in $\mathrm{DCM}(268 \mathrm{~mL})$ were added $\mathrm{N}, \mathrm{O}$-dimethylhydroxylamine hydrochloride $(5.5 \mathrm{~g}, 56.4 \mathrm{mmol}), 4$-methylmorpholine $(6.2 \mathrm{~mL}$, $56.4 \mathrm{mmol}$ ) and 1,(3-dimethylaminopropyl)-3-ethylcarbodiimide hydrochloride ( $10.8 \mathrm{~g}, 56.4 \mathrm{mmol})$. After $2 \mathrm{~h}$ of stirring at room temperature, the medium was washed with aqueous $\mathrm{HCl}(1 \mathrm{~N}$, two times), with brine, dried over sodium sulphate, filtered and concentrated in vacuo. Purification of the residue by chromatography on silica gel (cyclohexane-EtOAc, gradient of $8 / 2$ to $6 / 4$ ) afforded the title compound (22.6 g, quantitative): $\mathrm{LCMS}\left(t_{\mathrm{R}}=3.75 \mathrm{~min}\right.$, purity $\left.=100 \%\right), \mathrm{ESI}^{+}$ $\mathrm{m} / z$ 469.2 $(\mathrm{M}+\mathrm{H})^{+} ;{ }^{1} \mathrm{H} \mathrm{NMR}\left(\mathrm{CDCl}_{3}\right) \delta 7.75(\mathrm{~d}, J=7.5 \mathrm{~Hz}, 2 \mathrm{H}), 7.57-7.60$ $(\mathrm{m}, 2 \mathrm{H}), 7.39(\mathrm{t}, J=7.5 \mathrm{~Hz}, 2 \mathrm{H}), 7.30(\mathrm{td}, J=7.5 \mathrm{~Hz}$ and $1.0 \mathrm{~Hz}, 2 \mathrm{H}), 5.95$ $(\mathrm{d}, J=7.8 \mathrm{~Hz}, 1 \mathrm{H}), 4.40-4.35(\mathrm{~m}, 3 \mathrm{H}), 4.21(\mathrm{~m}, 1 \mathrm{H}), 3.70(\mathrm{~s}, 3 \mathrm{H}), 3.21(\mathrm{~s}$, $3 \mathrm{H}), 2.97-2.94(\mathrm{~m}, 1 \mathrm{H}), 2.73-2.64(\mathrm{~m}, 2 \mathrm{H}), 2.61(\mathrm{dd}, J=15.7 \mathrm{~Hz}$ and $7.0 \mathrm{~Hz}, 1 \mathrm{H}), 1.44(\mathrm{~s}, 9 \mathrm{H})$.

\subsection{2. tert-Butyl (R)-3-amino-5-(methoxy(methyl)amino)-5-oxo-} pentanoate

To a cooled $\left(0^{\circ} \mathrm{C}\right)$ solution of tert-butyl $(R)-3-((((9 H$-fluoren-9- yl)methoxy)carbonyl)amino)-5-(methoxy(methyl)amino)-5oxopentanoate $(22.6 \mathrm{~g}, 48.2 \mathrm{mmol})$ in DCM $(377 \mathrm{~mL})$ was added dropwise DBU ( $7.92 \mathrm{~mL}, 5.0 \mathrm{mmol}$ ). After $1 \mathrm{~h} 30$ of stirring at room temperature, the medium was concentrated under vacuum. Purification of the residue by chromatography on silica gel (DCM $\mathrm{MeOH} / \mathrm{NH}_{3}(7 \mathrm{~N})$, gradient of $97 / 3$ to $\left.95 / 5\right)$ afforded the title compound $\left(8.11 \mathrm{~g}, 68 \%\right.$, as yellow oil): ${ }^{1} \mathrm{H}$ NMR $\left(\mathrm{CDCl}_{3}\right) \delta 3.70-3.63(\mathrm{~m}$, $1 \mathrm{H}), 3.68(\mathrm{~s}, 3 \mathrm{H}), 3.17(\mathrm{~s}, 3 \mathrm{H}), 2.64-2.60(\mathrm{~m}, 1 \mathrm{H}), 2.57-2.52(\mathrm{~m}, 1 \mathrm{H})$, $2.45(\mathrm{dd}, J=15.8 \mathrm{~Hz}$ and $5.0 \mathrm{~Hz}, 1 \mathrm{H}), 2.39(\mathrm{dd}, J=15.8 \mathrm{~Hz}$ and $8.0 \mathrm{~Hz}$, $1 \mathrm{H}), 1.45(\mathrm{~s}, 9 \mathrm{H})$.

\subsection{3. tert-Butyl (R)-3-(((allyloxy)carbonyl)amino)-5-(methoxy-} methyl)amino)-5-oxo-pentanoate

To a cooled $\left(4{ }^{\circ} \mathrm{C}\right)$ solution of tert-butyl (R)-3-amino-5(methoxy(methyl)amino)-5-oxopentanoate $(8.11 \mathrm{~g}, 32.9 \mathrm{mmol})$ in $\mathrm{THF} / \mathrm{H}_{2} \mathrm{O}(20 \mathrm{~mL} / 60 \mathrm{~mL})$ were added sodium bicarbonate $(41.1 \mathrm{~g}$, $131.6 \mathrm{mmol})$ and allyl chloroformate $(6.30 \mathrm{~mL}, 59.2 \mathrm{mmol})$. After $1 \mathrm{~h}$ 30 of stirring at room temperature, the medium was extracted with EtOAc $(3 \times 20 \mathrm{~mL})$. The combined organic layer was dried over sodium sulphate, filtered and concentrated in vacuo. Purification of the residue by chromatography on silica gel (cyclohexane-EtOAc, gradient of $8 / 2$ to $6 / 4$ ) afforded the title compound $(9.58 \mathrm{~g}, 88 \%$ ) as a colorless oil: ${ }^{1} \mathrm{H}$ NMR $\left(\mathrm{CDCl}_{3}\right) \delta 5.93-5.83(\mathrm{~m}, 2 \mathrm{H}), 5.29(\mathrm{~d}$, $J=17.3 \mathrm{~Hz}, 1 \mathrm{H}), 5.18(\mathrm{~m}, 1 \mathrm{H}), 4.54(\mathrm{~m}, 2 \mathrm{H}), 4.32-4.36(\mathrm{~m}, 1 \mathrm{H}), 3.67$ $(\mathrm{s}, 3 \mathrm{H}), 3.17(\mathrm{~s}, 3 \mathrm{H}), 2.91-2.86(\mathrm{~m}, 1 \mathrm{H}), 2.72-2.65(\mathrm{~m}, 2 \mathrm{H}), 2.59(\mathrm{dd}$, $J=15.8 \mathrm{~Hz}$ and $6.9 \mathrm{~Hz}, 1 \mathrm{H}), 1.44(\mathrm{~s}, 9 \mathrm{H})$.

\subsection{4. tert-Butyl (R)-3-(((allyloxy)carbonyl)amino)-5-oxo- pentanoate}

A solution of lithium aluminium hydride $(2 \mathrm{~N}$ in THF, $5.90 \mathrm{~mL}$, $11.8 \mathrm{mmol})$ was added dropwise to a cooled $\left(-78^{\circ} \mathrm{C}\right)$ solution of tert-butyl (R)-3-(((allyloxy)carbonyl)amino)-5-(methoxy-(methyl) amino)-5-oxopentanoate $(6.0 \mathrm{~g}, 18.1 \mathrm{mmol})$ in anhydrous THF $(80 \mathrm{~mL})$. The mixture was stirred at $-78^{\circ} \mathrm{C}$ for $2 \mathrm{~h} 30$, then aqueous $\mathrm{HCl}(1 \mathrm{~N})$ was slowly added to the medium, and the temperature was allowed to warm to $0{ }^{\circ} \mathrm{C}$. The mixture was diluted with EtOAc. The aqueous layer was extracted (EtOAc, two times). The combined organic layer was washed with aqueous $\mathrm{HCl}(1 \mathrm{~N})$, with brine, dried over sodium sulphate, filtered and concentrated in vacuo to afford the title compound $(4.7 \mathrm{~g}, 95 \%$, as pale yellow oil $):{ }^{1} \mathrm{H} \mathrm{NMR}\left(\mathrm{CDCl}_{3}\right)$ $\delta 9.75(\mathrm{t}, J=1.4 \mathrm{~Hz}, 1 \mathrm{H}), 5.94-5.86(\mathrm{~m}, 1 \mathrm{H}), 5.48(\mathrm{br} \mathrm{s}, 1 \mathrm{H}), 5.29(\mathrm{~m}$, $1 \mathrm{H}), 5.20(\mathrm{~m}, 1 \mathrm{H}), 4.54(\mathrm{~d}, J=4.9 \mathrm{~Hz}, 2 \mathrm{H}), 4.37-4.40(\mathrm{~m}, 1 \mathrm{H}), 2.83$ (dd, $J=17.5 \mathrm{~Hz}$ and $5.5 \mathrm{~Hz}, 1 \mathrm{H}$ ), 2.74 (ddd, $J=17.5 \mathrm{~Hz}, 6.0 \mathrm{~Hz}$ and $1.4 \mathrm{~Hz}, 1 \mathrm{H}), 2.58(\mathrm{~d}, J=5.8 \mathrm{~Hz}, 2 \mathrm{H}), 1.44(\mathrm{~s}, 9 \mathrm{H})$.

\subsection{5. tert-Butyl (R)-3-(((allyloxy)carbonyl)amino)-5,5-diethoxy- pentanoate}

To a freshly prepared solution of tert-butyl ( $R)-3-((($ allyloxy) carbonyl)amino)-5-oxopentanoate $(4.7 \mathrm{~g}, 17.3 \mathrm{mmol})$ in absolute ethanol $(20 \mathrm{~mL})$ were added, under Argon atmosphere, triethyl orthoformate ( $11.5 \mathrm{~mL}, 69.3 \mathrm{mmol}), p$-toluenesulfonic acid $(90 \mathrm{mg}$, $0.52 \mathrm{mmol}$ ) and $3 \AA$ molecular sieves. After stirring at room temperature overnight, the mixture was filtered over a pad of Celite and rinsed with EtOH. The filtrate was concentrated under reduced pressure and co-evaporated with toluene (three times). Purification of the residue by flash chromatography on silica gel (cyclohexaneEtOAc, gradient of 9/1 to 8/2) afforded the title compound (4.85 g, $81 \%$, as pale yellow oil): ${ }^{1} \mathrm{H}$ NMR $\left(400 \mathrm{MHz}\right.$, DMSO- $\left.d_{6}\right) \delta 7.13(\mathrm{~d}$, $J=8.8 \mathrm{~Hz}, 1 \mathrm{H}), 5.92-5.85(\mathrm{~m}, 1 \mathrm{H}), 5.27-5.24(\mathrm{~m}, 1 \mathrm{H}), 5.17-5.14(\mathrm{~m}$, $1 \mathrm{H}), 4.47-4.44(\mathrm{~m}, 3 \mathrm{H}), 3.90-3.84(\mathrm{~m}, 1 \mathrm{H}), 3.59-3.50(\mathrm{~m}, 2 \mathrm{H})$, 3.43-3.73 (m, 2H), 2.36-2.28 (m, 2H), $1.65(\mathrm{~m}, 2 \mathrm{H}), 1.37(\mathrm{~s}, 9 \mathrm{H}), 1.09$ $(\mathrm{m}, 6 \mathrm{H}) ;{ }^{13} \mathrm{C}$ NMR $(101 \mathrm{MHz}$, DMSO-d $) \delta 169.97,155.13,133.83$, 116.63, 100.06, 79.73, 64.09, 61.11, 60.48, 45.22, 41.12, 38.26, 27.67, 15.29; $[\alpha]_{\mathrm{D}}^{20}=+13.4^{\circ}(\mathrm{c}=10 \mathrm{~g} / \mathrm{L}, \mathrm{EtOH})$. HRMS: $(\mathrm{M}+\mathrm{H})^{+}$calculated for $\mathrm{C}_{17} \mathrm{H}_{31} \mathrm{NO}_{6}$ 345.2151; found $368.2083(\mathrm{M}+\mathrm{Na})^{+}, 713.4276$ 
$(2 \mathrm{M}+\mathrm{Na})^{+}, 244.1206$ cyclized warhead eg., $\left(\mathrm{M}\left(-\mathrm{OEt},-\mathrm{C}_{4} \mathrm{H}_{9}\right)+\mathrm{H}\right)^{+}$.

4.7.6. tert-Butyl (R)-5,5-Diethoxy-3-(1-(3-isopropyl-5-(2-methyl4-(naphthalen-2-ylamino)-benzamido)-2,6-dioxo-3,6-

dihydropyrimidin-1(2H)-yl)cyclopropane-1-carboxamido)-

pentanoate

Step 1: To a stirred solution 1-\{3-isopropyl-5-[2-methyl-4(naphthalen-2-ylamino)-benzoylamino]-2,6-dioxo-3,6-dihydro$2 \mathrm{H}$-pyrimidin-1-yl\}-cyclopropanecarboxylic acid (100 $\mathrm{mg}$, $0.20 \mathrm{mmol}, 1.00$ eq.) and $N$-methylmorpholine $(25.7 \mu \mathrm{L}, 0.23 \mathrm{mmol}$, 1.20 eq.) in a mixture of DCM $(700 \mu \mathrm{L})$ and DMF $(1.00 \mathrm{~mL})$, was added HATU ( $89.0 \mathrm{mg}, 0.23 \mathrm{mmol}, 1.20$ eq.) and the reaction mixture was stirred at room temperature for $30 \mathrm{~min}$.

Step 2: In a separate flask, a solution of ( $R$ )-3-allyloxycarbonylamino-5,5-diethoxy-pentanoic acid tert-butyl ester (101 $\mathrm{mg}, 0.29 \mathrm{mmol}, 1.50$ eq.) and 1,3-dimethylbarbituric acid ( $45.7 \mathrm{mg}, 0.29 \mathrm{mmol}, 1.50$ eq.) in a mixture of DCM $(700 \mu \mathrm{L})$ and DMF $(500 \mu \mathrm{L})$ was degassed and purged with nitrogen then tetrakis(triphenylphosphine)palladium $(0) \quad(9.02 \mathrm{mg}, \quad 0.01 \mathrm{mmol}, 0.04$ eq.) was added. The reaction mixture was stirred at r.t. for $30 \mathrm{~min}$. Step 3: The solution from the Alloc deprotection containing tertbutyl $(R)$-3-amino-5,5-diethoxypentanoate was added dropwise to the activated ester formed in Step 1 and the resulting solution was stirred for $2 \mathrm{~h}$ at r.t. The reaction mixture was diluted with EtOAc $(30 \mathrm{~mL})$ and washed with a saturated aqueous solution of $\mathrm{NaHCO}_{3}$. The aqueous phase was washed with $\operatorname{EtOAc}(2 \times 10 \mathrm{~mL})$. The organic phases were combined, dried over $\mathrm{MgSO}_{4}$ and concentrated to dryness. The residue was purified by flash chromatography (silica gel, gradient of $0-100 \%$ EtOAc in heptane) to afford tert-butyl $(R)$ 5,5-diethoxy-3-(1-(3-isopropyl-5-(2-methyl-4-(naphthalen-2ylamino)-benzamido)-2,6-dioxo-3,6-dihydropyrimidin-1(2H)-yl) cyclopropane-1-carboxamido)-pentanoate (140 mg, 95\%) as a yellow solid: $\mathrm{LCMS}\left(t_{\mathrm{R}}=1.44 \mathrm{~min}\right.$, purity $\left.=100 \%\right)$, $\mathrm{MS} \mathrm{ES}^{-} \mathrm{m} / z 754.48$ $(\mathrm{M}-\mathrm{H})^{-} ;{ }^{1} \mathrm{H}$ NMR $\left(400 \mathrm{MHz}\right.$, DMSO- $\left.d_{6}\right) \delta 8.83(\mathrm{~d}, J=6.3 \mathrm{~Hz}, 1 \mathrm{H}), 8.72$ $(\mathrm{s}, 1 \mathrm{H}), 8.42(\mathrm{~d}, J=6.3 \mathrm{~Hz}, 1 \mathrm{H}), 7.89-7.71(\mathrm{~m}, 3 \mathrm{H}), 7.69-7.55(\mathrm{~m}, 2 \mathrm{H})$, 7.53-7.38 (m, 2H), 7.38-7.28 (m, 2H), 7.13-6.99 (m, 2H), 4.77 (p, $J=6.7 \mathrm{~Hz}, 1 \mathrm{H}), 4.46(\mathrm{dd}, J=8.1,3.1 \mathrm{~Hz}, 1 \mathrm{H}), 3.49$ (overlapping $\mathrm{m}, 4 \mathrm{H})$, $2.65-2.53(\mathrm{~m}, 1 \mathrm{H}), 2.44(\mathrm{~s}, 3 \mathrm{H}), 2.39-2.17(\mathrm{~m}, 1 \mathrm{H}), 1.81-1.50(\mathrm{~m}, 2 \mathrm{H})$, $1.47-1.18$ (overlapping signals, $17 \mathrm{H}), 1.10(\mathrm{~m}, 6 \mathrm{H})$.

4.7.7. $\mathrm{N}-(3-(1-(((4 S)-2-h y d r o x y-6-o x o t e t r a h y d r o-2 H-p y r a n-4-y l)$ carbamoyl)cyclopropyl)-1-isopropyl-2,4-dioxo-1,2,3,4tetrahydropyrimidin-5-yl)-2-methyl-4-(naphthalen-2-ylamino)benzamide (37)

To a stirred solution of $(R)$-5,5-diethoxy-3-[(1-\{3-isopropyl-5[2-methyl-4-(naphthalen-2-ylamino)-benzoylamino]-2,6-dioxo3,6-dihydro-2H-pyrimidin-1-yl\}-cyclopropanecarbonyl)-amino]pentanoic acid tert-butyl ester ( $200 \mathrm{mg}, 0.26 \mathrm{mmol}, 1.00 \mathrm{eq}$.$) in$ DCM $(6.00 \mathrm{~mL})$ at $0{ }^{\circ} \mathrm{C}$, was added TFA $(6.00 \mathrm{~mL}, 78.4 \mathrm{mmol})$ dropwise over $2 \mathrm{~min}$. The reaction mixture was stirred at r.t. for $2 \mathrm{~h}$. The reaction mixture was carefully added to a mixture of ice and a saturated solution of $\mathrm{NaHCO}_{3}(\mathrm{aq})$. The $\mathrm{pH}$ of the reaction mixture was brought up to 8 with $1 \mathrm{M} \mathrm{NaOH}$ (aq) then lowered to 5 with $\mathrm{AcOH}$ and the product extracted with $\mathrm{DCM}(2 \times 10 \mathrm{~mL})$. The organic extracts were combined, washed with water $(2 \times 2 \mathrm{~mL})$, dried over $\mathrm{MgSO}_{4}$ and concentrated to dryness. The residue was purified by flash chromatography (silica gel, gradient of $2-10 \% \mathrm{MeOH}$ in DCM) to afford a yellow solid. The solid was triturated with heptane and stirred overnight, recuperated by filtration and dried to a constant weight to afford $\mathrm{N}$-(3-(1-(((4S)-2-hydroxy-6-oxotetrahydro- $2 \mathrm{H}$ pyran-4-yl)carbamoyl)cyclopropyl)-1-isopropyl-2,4-dioxo-1,2,3,4tetrahydropyrimidin-5-yl)-2-methyl-4-(naphthalen-2-ylamino)benzamide (134 mg, $80 \%$ ) as an off-white solid: LCMS $\left(t_{\mathrm{R}}=6.57 \mathrm{~min}\right.$, purity $\left.=98.4 \%\right), \mathrm{ESI}^{+} \mathrm{m} / z 626.25(\mathrm{M}+\mathrm{H})^{+} ;{ }^{1} \mathrm{H} \mathrm{NMR}$ $\left(400 \mathrm{MHz}, \mathrm{DMSO}-d_{6}\right) \delta 9.52(\mathrm{~s}, 1 \mathrm{H}), 8.84(\mathrm{~s}, 1 \mathrm{H}), 8.72(\mathrm{~s}, 1 \mathrm{H}), 8.41(\mathrm{~s}$,
1H), $7.99(\mathrm{~s}, 1 \mathrm{H}), 7.71-7.88(\mathrm{~m}, 3 \mathrm{H}), 7.58(\mathrm{~d}, J=2.4 \mathrm{~Hz}, 1 \mathrm{H}), 7.50(\mathrm{~d}$, $J=8.6 \mathrm{~Hz}, 1 \mathrm{H}), 7.43$ (ddd, $J=8.2,6.8,1.3 \mathrm{~Hz}, 1 \mathrm{H}), 7.26-7.37(\mathrm{~m}, 2 \mathrm{H})$, 6.94-7.03 (m, 2H), $4.76(\mathrm{~s}, 1 \mathrm{H}), 4.46(\mathrm{~s}, 1 \mathrm{H}), 2.30-2.50(\mathrm{~m}, 2 \mathrm{H}), 2.44$ (s, 3H), $2.38(\mathrm{~d}, J=10.9 \mathrm{~Hz}, 2 \mathrm{H}), 1.61(\mathrm{~m}, 2 \mathrm{H}), 1.28-1.38(\mathrm{~m}, 6 \mathrm{H}), 1.11$ $(\mathrm{s}, 2 \mathrm{H}) ;{ }^{13} \mathrm{C}$ NMR $\left(101 \mathrm{MHz}\right.$, DMSO-d $\left.d_{6}\right) \delta 169.04,167.23,160.07$, 149.74, 148.66, 145.39, 140.14, 138.32, 135.06, 134.26, 129.48, 128.99, $128.72,128.33,127.51,126.51,126.40,126.10,123.46,120.60,118.24$, $113.92,112.85,111.52,48.08,42.61,37.37,31.30,28.41,22.14,20.59$, 14.00; HRMS $(\mathrm{M}+\mathrm{H})^{+}$calculated for $\mathrm{C}_{34} \mathrm{H}_{35} \mathrm{~N}_{5} \mathrm{O}_{7}$ 626.2536; found 626.2605

4.8. N-(3-(1-(((4S)-2-Hydroxy-6-oxotetrahydro-2H-pyran-4-yl) carbamoyl)cyclopropyl)-1-isopropyl-2,4-dioxo-1,2,3,4tetrahydropyrimidin-5-yl)-2-methyl-4-(naphthalen-2-ylamino) benzamide (38) was prepared in 7 steps

4.8.1. tert-Butyl (S)-3-((((9H-fluoren-9-yl)methoxy)-carbonyl)amino)-5-(methoxy (methyl)amino)-5-oxopentanoate

To a cooled solution of $(S)-3-((((9 H-$ fluoren-9-yl)methoxy $)$ carbonyl)amino)-5-(tert-butoxy)-5-oxopentanoic acid (5.0 g, $11.7 \mathrm{mmol})$ in DCM $(67 \mathrm{~mL})$ were added $\mathrm{N}, \mathrm{O}$-dimethylhydroxylamine hydrochloride $(1.38 \mathrm{~g}, 14.1 \mathrm{mmol})$, 4-methylmorpholine (1.55 mL, $14.1 \mathrm{mmol})$ and 1,(3-dimethylaminopropyl)-3ethylcarbodiimide hydrochloride $(2.70 \mathrm{~g}, 14.1 \mathrm{mmol})$. After $2 \mathrm{~h} 30$ of stirring at room temperature, the medium was washed with aqueous $\mathrm{HCl}$ ( $1 \mathrm{~N}$, three times), with saturated aqueous sodium bicarbonate, with brine, dried over sodium sulphate, filtered and concentrated in vacuo to afford the title compound $(4.98 \mathrm{~g}, 90 \%)$ as a pale yellow paste: $\mathrm{LCMS}\left(t_{\mathrm{R}}=3.75 \mathrm{~min}\right.$, purity $\left.=100 \%\right), \mathrm{ESI}^{+} \mathrm{m} / \mathrm{z}$ $469.2(\mathrm{M}+\mathrm{H})^{+} ;{ }^{1} \mathrm{H}$ NMR $\left(\mathrm{CDCl}_{3}\right) \delta 7.75(\mathrm{~d}, J=7.5 \mathrm{~Hz}, 2 \mathrm{H}), 7.60-7.57$ $(\mathrm{m}, 2 \mathrm{H}), 7.39(\mathrm{t}, J=7.5 \mathrm{~Hz}, 2 \mathrm{H}), 7.30(\mathrm{td}, J=7.5 \mathrm{~Hz}$ and $1.0 \mathrm{~Hz}, 2 \mathrm{H})$, $5.95(\mathrm{~d}, J=7.8 \mathrm{~Hz}, 1 \mathrm{H}), 4.40-4.35(\mathrm{~m}, 3 \mathrm{H}), 4.21(\mathrm{~m}, 1 \mathrm{H}), 3.70(\mathrm{~s}, 3 \mathrm{H})$, $3.21(\mathrm{~s}, 3 \mathrm{H}), 2.97-2.94(\mathrm{~m}, 1 \mathrm{H}), 2.73-2.68(\mathrm{~m}, 2 \mathrm{H}), 2.61$ (dd, $J=15.7 \mathrm{~Hz}$ and $7.0 \mathrm{~Hz}, 1 \mathrm{H}), 1.44(\mathrm{~s}, 9 \mathrm{H})$.

\subsection{2. tert-Butyl (S)-3-amino-5-(methoxy(methyl)amino)-5- oxopentanoate}

To a solution of tert-butyl (S)-3-((((9H-fluoren-9-yl)methoxy) carbonyl)amino)-5-(methoxy(methyl)amino)-5-oxopentanoate $(4.95 \mathrm{~g}, 10.5 \mathrm{mmol})$ in DCM $(80 \mathrm{~mL})$ was added dropwise DBU $(1.73 \mathrm{~mL}, 11.6 \mathrm{mmol})$. After $2 \mathrm{~h} 30$ of stirring at room temperature, the medium was concentrated under vacuum. Purification of the residue by chromatography on silica gel (DCM - MeOH/NH3 (7 N), 95/5) afforded the title compound $(2.45 \mathrm{~g}, 75 \%)$ as a yellow oil; ${ }^{1} \mathrm{H}$ NMR $\left(\mathrm{CDCl}_{3}, 500 \mathrm{MHz}\right) \delta 3.63-3.68(\mathrm{~m}, 1 \mathrm{H}), 3.68(\mathrm{~s}, 3 \mathrm{H}), 3.17(\mathrm{~s}$, $3 \mathrm{H}), 2.64-2.60(\mathrm{~m}, 1 \mathrm{H}), 2.57-2.52(\mathrm{~m}, 1 \mathrm{H}), 2.45(\mathrm{dd}, J=15.8 \mathrm{~Hz}$ and $5.0 \mathrm{~Hz}, 1 \mathrm{H}), 2.39(\mathrm{dd}, J=15.8 \mathrm{~Hz}$ and $8.0 \mathrm{~Hz}, 1 \mathrm{H}), 1.45(\mathrm{~s}, 9 \mathrm{H})$.

\subsection{3. tert-Butyl (S)-3-(((allyloxy)carbonyl)amino)-5-(methoxy-} (methyl)amino)-5-oxopentanoate

To a cooled $\left(4{ }^{\circ} \mathrm{C}\right)$ solution of tert-butyl (S)-3-amino-5(methoxy(methyl)amino)-5-oxopentanoate $(2.45 \mathrm{~g}, 9.95 \mathrm{mmol})$ in $\mathrm{THF} / \mathrm{H}_{2} \mathrm{O}(6 \mathrm{~mL} / 18 \mathrm{~mL})$ were added sodium bicarbonate $(3.34 \mathrm{~g}$, $39.79 \mathrm{mmol})$ and allyl chloroformate $(1.90 \mathrm{~mL}, 17.9 \mathrm{mmol})$. After $2 \mathrm{~h} 30$ of stirring at room temperature, the medium was extracted with EtOAc (three times). The combined organic layer was dried over sodium sulphate, filtered and concentrated in vacuo. Purification of the residue by chromatography on silica gel (cyclohexaneEtOAc, gradient of $8 / 2$ to $4 / 6$ ) afforded the title compound ( $1.32 \mathrm{~g}$, $40 \%)$ as a colorless oil: $\operatorname{LCMS}\left(t_{\mathrm{R}}=3.00 \mathrm{~min}\right.$, purity $\left.=100 \%\right), \mathrm{ESI}^{+} \mathrm{m} /$ $z 331.1(\mathrm{M}+\mathrm{H})^{+} ;{ }^{1} \mathrm{H}$ NMR $\left(\mathrm{CDCl}_{3}\right) \delta 5.83-5.93(\mathrm{~m}, 2 \mathrm{H}), 5.29(\mathrm{~d}$, $J=17.3 \mathrm{~Hz}, 1 \mathrm{H}), 5.18(\mathrm{~m}, 1 \mathrm{H}), 4.54(\mathrm{~m}, 2 \mathrm{H}), 4.36-4.32(\mathrm{~m}, 1 \mathrm{H}), 3.67$ $(\mathrm{s}, 3 \mathrm{H}), 3.17(\mathrm{~s}, 3 \mathrm{H}), 2.91-2.86(\mathrm{~m}, 1 \mathrm{H}), 2.72-2.65(\mathrm{~m}, 2 \mathrm{H}), 2.59$ (dd, $J=15.8 \mathrm{~Hz}$ and $6.9 \mathrm{~Hz}, 1 \mathrm{H}), 1.44(\mathrm{~s}, 9 \mathrm{H})$. 
4.8.4. tert-Butyl (S)-3-(((allyloxy)carbonyl)amino)-5-oxopentanoate

A solution of lithium aluminium hydride $(2 \mathrm{~N}$ in THF, $1.28 \mathrm{~mL}$, $2.56 \mathrm{mmol})$ was added dropwise to a cooled $\left(-78^{\circ} \mathrm{C}\right)$ solution of tert-butyl (S)-3-(((allyloxy)carbonyl)amino)-5-(methoxy-(methyl) amino)-5-oxopentanoate $(1.30 \mathrm{~g}, 3.93 \mathrm{mmol})$ in anhydrous THF $(16 \mathrm{~mL})$. The mixture was stirred at $-78^{\circ} \mathrm{C}$ for $3 \mathrm{~h}$, then aqueous $\mathrm{HCl}$ $(1 \mathrm{~N})$ was slowly added to the medium, and the temperature was allowed to warm to $0{ }^{\circ} \mathrm{C}$. The mixture was diluted with EtOAc. The aqueous layer was extracted (EtOAc, two times). The combined organic layer was washed with aqueous $\mathrm{HCl}(1 \mathrm{~N})$, with brine, dried over sodium sulphate, filtered and concentrated in vacuo to afford the title compound $(1.05 \mathrm{~g}, 98 \%)$ as a pale yellow oil: ${ }^{1} \mathrm{H}$ NMR $\left(\mathrm{CDCl}_{3}\right) \delta 9.75(\mathrm{t}, J=1.4 \mathrm{~Hz}, 1 \mathrm{H}), 5.86-5.94(\mathrm{~m}, 1 \mathrm{H}), 5.48($ br s, $1 \mathrm{H})$, $5.29(\mathrm{~m}, 1 \mathrm{H}), 5.20(\mathrm{~m}, 1 \mathrm{H}), 4.54(\mathrm{~d}, J=4.9 \mathrm{~Hz}, 2 \mathrm{H}), 4.40-4.37(\mathrm{~m}$, $1 \mathrm{H}), 2.83(\mathrm{dd}, J=17.5 \mathrm{~Hz}$ and $5.5 \mathrm{~Hz}, 1 \mathrm{H}), 2.74$ (ddd, $J=17.5 \mathrm{~Hz}$, $6.0 \mathrm{~Hz}$ and $1.4 \mathrm{~Hz}, 1 \mathrm{H}), 2.58(\mathrm{~d}, J=5.8 \mathrm{~Hz}, 2 \mathrm{H}), 1.44(\mathrm{~s}, 9 \mathrm{H})$.

4.8.5. tert-Butyl (S)-3-(((allyloxy)carbonyl)amino)-5,5-diethoxypentanoate

To a freshly prepared solution of tert-butyl (S)-3-(((allyloxy) carbonyl)amino)-5-oxopentanoate $(1.05 \mathrm{~g}, 3.87 \mathrm{mmol})$ in absolute ethanol (3.5 mL) were added, under an argon atmosphere, triethyl orthoformate ( $1.61 \mathrm{~mL}, 9.68 \mathrm{mmol}), p$-toluenesulfonic acid (13 mg, $0.077 \mathrm{mmol}$ ) and $3 \AA$ molecular sieves. After stirring at room temperature overnight, the mixture was filtered over a pad of celite and rinsed with $\mathrm{EtOH}$. The filtrate was concentrated under reduced pressure and co-evaporated with toluene (three times). Purification of the residue by flash chromatography on silica gel (cyclohexaneEtOAc, gradient of 9/1 to 75/25) afforded the title compound $(1.07 \mathrm{~g}$, $80 \%$ ) as a pale yellow oil; LCMS ( $t_{\mathrm{R}}=3.53 \mathrm{~min}$, purity $\left.=100 \%\right), \mathrm{ESI}^{+}$ $\mathrm{m} / \mathrm{z} 300.1(\mathrm{M}(-\mathrm{OEt})+\mathrm{H}){ }^{+} ;{ }^{1} \mathrm{H}$ NMR (DMSO- $\left.d_{6}, 400 \mathrm{MHz}\right) \delta 7.13(\mathrm{~d}$, $J=8.8 \mathrm{~Hz}, 1 \mathrm{H}), 5.92-5.84(\mathrm{~m}, 1 \mathrm{H}), 5.27-5.24(\mathrm{~m}, 1 \mathrm{H}), 5.17-5.14(\mathrm{~m}$, $1 \mathrm{H}), 4.47-4.44(\mathrm{~m}, 3 \mathrm{H}), 3.90-3.84(\mathrm{~m}, 1 \mathrm{H}), 3.59-3.50(\mathrm{~m}, 2 \mathrm{H})$, 3.43-3.37 (m, 2H), 2.36-2.28 (m, 2H), $1.65(\mathrm{~m}, 2 \mathrm{H}), 1.37(\mathrm{~s}, 9 \mathrm{H}), 1.09$ $(\mathrm{m}, 6 \mathrm{H}) ;{ }^{13} \mathrm{C}$ NMR (101 MHz, DMSO- $\left.d_{6}\right) \delta 169.97,155.13,133.83$, 116.63, 100.06, 79.73, 64.09, 61.11, 60.48, 45.22, 41.12, 38.26, 27.67, 15.29; $[\alpha]_{\mathrm{D}}^{20}=-12.8^{\circ}(\mathrm{c}=10 \mathrm{~g} / \mathrm{L}, \mathrm{EtOH}) ;$ HRMS: $(\mathrm{M}+\mathrm{H})+$ calculated for $\mathrm{C}_{17} \mathrm{H}_{31} \mathrm{NO}_{6}$ 345.2151; found $368.2082(\mathrm{M}+\mathrm{Na})^{+}, 713.4275$ $(2 \mathrm{M}+\mathrm{Na})^{+}, 244.1205$ cyclized warhead eg., $\left(\mathrm{M}\left(-\mathrm{OEt},-\mathrm{C}_{4} \mathrm{H}_{9}\right)+\mathrm{H}\right)^{+}$.

4.8.6. tert-Butyl (S)-5,5-diethoxy-3-(1-(3-isopropyl-5-(2-methyl-4(naphthalen-2-ylamino)-benzamido)-2,6-dioxo-3,6-

dihydropyrimidin-1(2H)-yl)cyclopropane-1-carboxamido)pentanoate

Step 1: To a stirred solution of 1-\{3-Isopropyl-5-[2-methyl-4(naphthalen-2-ylamino)-benzoylamino]-2,6-dioxo-3,6-dihydro$2 \mathrm{H}$-pyrimidin-1-yl\}-cyclopropanecarboxylic acid (100 mg, $0.20 \mathrm{mmol}, 1.00$ eq.) and $N$-methylmorpholine $(25.7 \mu \mathrm{L}, 0.23 \mathrm{mmol}$, 1.20 eq.) in a mixture of DCM $(700 \mu \mathrm{L})$ and $\mathrm{DMF}(1.00 \mathrm{~mL})$, was added HATU $(89.0 \mathrm{mg}, 0.23 \mathrm{mmol}, 1.20$ eq.) and the reaction mixture was stirred at room temperature for $30 \mathrm{~min}$.

Step 2: In parallel, a stirred solution of (S)-3-allyloxycarbonylamino5,5-diethoxy-pentanoic acid tert-butyl ester ( $101 \mathrm{mg}, 0.29 \mathrm{mmol}, 1.50$ eq.) and 1,3-dimethylbarbituric acid ( $45.7 \mathrm{mg}, 0.29 \mathrm{mmol}, 1.50 \mathrm{eq}$.) in a mixture of DCM $(700 \mu \mathrm{L})$ and $\mathrm{DMF}(500 \mu \mathrm{L})$ was degassed and purged with nitrogen then tetrakis(triphenylphosphine)palladium $(0)$ (9.02 $\mathrm{mg}, 0.01 \mathrm{mmol}, 0.04$ eq.) was added. The reaction mixture was agitated for $30 \mathrm{~min}$.

Step 3: The solution containing tert-butyl (S)-3-amino-5,5diethoxypentanoate was added dropwise to the pre-activated ester for Step 1 and the resulting solution was stirred for $2 \mathrm{~h}$ at r.t. The reaction mixture was diluted with EtOAc $(50 \mathrm{~mL})$ and washed with a saturated aqueous solution of $\mathrm{NaHCO}_{3}$. The aqueous phase was washed with EtOAc $(3 \times 10 \mathrm{~mL})$. The organic phases were combined, dried over $\mathrm{MgSO}_{4}$ and concentrated to dryness. The residue was purified by flash chromatography (silica gel, gradient of $0-100 \%$ EtOAc in heptane) to afford tert-butyl (S)-5,5-diethoxy-3(1-(3-isopropyl-5-(2-methyl-4-(naphthalen-2-ylamino)-benzamido)-2,6-dioxo-3,6-dihydropyrimidin-1(2H)-yl)cyclopropane-1carboxamido)-pentanoate (141 $\mathrm{mg}, 96 \%$ ) as a beige solid: LCMS $\left(t_{\mathrm{R}}=1.44 \mathrm{~min}\right.$, purity $\left.=100 \%\right), \mathrm{MS} \mathrm{ESI}^{-} \mathrm{m} / z 754.63(\mathrm{M}-\mathrm{H})^{-} ;{ }^{1} \mathrm{H}$ NMR $\left(400 \mathrm{MHz}, \mathrm{DMSO}-\mathrm{d}_{6}\right) \delta 8.83(\mathrm{~d}, J=6.4 \mathrm{~Hz}, 1 \mathrm{H}), 8.72(\mathrm{~s}, 1 \mathrm{H}), 8.42(\mathrm{~d}$, $J=6.4 \mathrm{~Hz}, 1 \mathrm{H}), 7.88-7.72(\mathrm{~m}, 3 \mathrm{H}), 7.69-7.52(\mathrm{~m}, 4 \mathrm{H}), 7.52-7.39(\mathrm{~m}$, $1 \mathrm{H}), 7.38-7.27(\mathrm{~m}, 2 \mathrm{H}), 7.12-7.00(\mathrm{~m}, 2 \mathrm{H}), 5.83-5.57(\mathrm{~m}, 1 \mathrm{H})$, 5.17-4.95 (m, 1H), 4.90-4.59 (m, 1H), $4.46(\mathrm{~m}, 1 \mathrm{H}), 4.16$ (overlapping $\mathrm{m}, 1 \mathrm{H}), 3.70-3.35$ (overlapping $\mathrm{m}, 4 \mathrm{H}), 2.75-2.55(\mathrm{~m}, 1 \mathrm{H})$, $2.44(\mathrm{~s}, 3 \mathrm{H}), 2.37-2.15(\mathrm{~m}, 1 \mathrm{H}), 1.78-1.53(\mathrm{~m}, 2 \mathrm{H}), 1.46-1.18$ (overlapping $\mathrm{m}, 17 \mathrm{H}), 1.10(\mathrm{~m}, 6 \mathrm{H})$.

4.8.7. N-(3-(1-(((4S)-2-Hydroxy-6-oxotetrahydro-2H-pyran-4-yl) carbamoyl)cyclopropyl)-1-isopropyl-2,4-dioxo-1,2,3,4tetrahydropyrimidin-5-yl)-2-methyl-4-(naphthalen-2-ylamino) benzamide (38)

To a stirred solution of tert-butyl (S)-5,5-diethoxy-3-(1-(3isopropyl-5-(2-methyl-4-(naphthalen-2-ylamino)-benzamido)2,6-dioxo-3,6-dihydropyrimidin-1(2H)-yl)cyclopropane-1carboxamido)-pentanoate $(260 \mathrm{mg}, 0.34 \mathrm{mmol}, 1.00$ eq.) in DCM $(7.8 \mathrm{~mL})$ was added TFA $(7.8 \mathrm{~mL})$ at $0{ }^{\circ} \mathrm{C}$. The reaction mixture is stirred at r.t. for $2 \mathrm{~h}$. The reaction mixture was carefully added to a mixture of ice and a saturated solution of $\mathrm{NaHCO}_{3}(\mathrm{aq})$. The $\mathrm{pH}$ of the reaction mixture was lowered to 5 with $\mathrm{AcOH}$ and the product extracted with DCM $(2 \times 5 \mathrm{~mL})$. The organic extracts were combined, washed with water $(2 \times 2 \mathrm{~mL})$, dried over $\mathrm{MgSO}_{4}$ and concentrated to dryness. The residue was purified by flash chromatography (silica gel, gradient of $2-10 \% \mathrm{MeOH}$ in DCM) to afford a yellow solid. The solid was triturated with heptane and stirred overnight, recuperated by filtration and dried to a constant weight to afford $\mathrm{N}$-(3-(1-(((4S)-2-hydroxy-6-oxotetrahydro-2H-pyran-4yl)carbamoyl)cyclopropyl)-1-isopropyl-2,4-dioxo-1,2,3,4-

tetrahydropyrimidin-5-yl)-2-methyl-4-(naphthalen-2 -ylamino) benzamide (129 mg, 59\%) as a pale yellow solid: LCMS $\left(t_{\mathrm{R}}=5.96 \mathrm{~min}\right.$, purity $\left.=98 \%\right)$, MS ESI ${ }^{+} \mathrm{m} / z 624.57(\mathrm{M}-\mathrm{H})^{-} ;{ }^{1} \mathrm{H}$ NMR $\left(400 \mathrm{MHz}, \mathrm{DMSO}-d_{6}\right) \delta 9.53(\mathrm{~s}, 1 \mathrm{H}), 8.84(\mathrm{~s}, 1 \mathrm{H}), 8.71(\mathrm{~s}, 1 \mathrm{H}), 8.40(\mathrm{~s}$, $1 \mathrm{H}), 7.70-7.90(\mathrm{~m}, 3 \mathrm{H}), 7.58(\mathrm{~d}, J=2.3 \mathrm{~Hz}, 1 \mathrm{H}), 7.50(\mathrm{~d}, J=8.5 \mathrm{~Hz}$, $1 \mathrm{H}), 7.42(\mathrm{~d}, J=7.4 \mathrm{~Hz}, 1 \mathrm{H}), 7.24-7.38(\mathrm{~m}, 2 \mathrm{H}), 6.94-7.13(\mathrm{~m}, 2 \mathrm{H})$, $4.76(\mathrm{~s}, 1 \mathrm{H}), 4.42(\mathrm{~s}, 1 \mathrm{H}), 2.40-2.50(\mathrm{~m}, 2 \mathrm{H}), 2.44(\mathrm{~s}, 3 \mathrm{H}), 2.32(\mathrm{~s}, 2 \mathrm{H})$, $1.69-1.81(\mathrm{~m}, 2 \mathrm{H}), 1.32(\mathrm{dd}, J=11.0,6.7 \mathrm{~Hz}, 6 \mathrm{H}), 1.11(\mathrm{~s}, 2 \mathrm{H}) ;{ }^{13} \mathrm{C}$ NMR $\left(101 \mathrm{MHz}\right.$, DMSO- $\left.d_{6}\right) \delta$ 169.04, 167.23, 160.07, 149.74, 148.66 , $145.39,140.14,138.32,135.06,134.26,129.48,128.99,128.72,128.33$, $127.51,126.51,126.40,126.10,123.46,120.60,118.24,113.92,112.85$, $111.52,48.08,42.61,37.37,31.30,28.41,22.14,20.59,14.00$; HRMS: $(\mathrm{M}+\mathrm{H})^{+}$calculated for $\mathrm{C}_{34} \mathrm{H}_{35} \mathrm{~N}_{5} \mathrm{O}_{7} 626.2536$; found, 626.2600.

4.9. $\mathrm{N}-(3-(1-(((3 S)-2-H y d r o x y-6-o x o t e t r a h y d r o-2 H-p y r a n-3-y l)$ amino)-1-oxopropan-2-yl)-1-isopropyl-2,4-dioxo-1,2,3,4tetrahydropyrimidin-5-yl)-2-methyl-4-(naphthalen-2-ylamino) benzamide (39) prepared in 6 steps

\subsection{1. (S)-2-(((Allyloxy)carbonyl)amino)-5-(tert-butoxy)-5-oxo- pentanoic acid}

To a cooled $\left(4^{\circ} \mathrm{C}\right)$ solution of L-glutamic acid 4-tert-butyl ester $(15.0 \mathrm{~g}, 73.8 \mathrm{mmol})$ in $\mathrm{THF} / \mathrm{H}_{2} \mathrm{O}(45 \mathrm{~mL} / 135 \mathrm{~mL})$ were added sodium bicarbonate $(24.8 \mathrm{~g}, 295.2 \mathrm{mmol}$ ) and allyl chloroformate $(14.12 \mathrm{~mL}$, $132.8 \mathrm{mmol}$ ). After $3 \mathrm{~h}$ of stirring at room temperature, the medium was acidified with aqueous $\mathrm{HCl}(6 \mathrm{~N})$ until obtaining $\mathrm{pH} 2$. The aqueous layer was extracted (EtOAc). The organic layer was dried over sodium sulphate, filtered and concentrated under reduced pressure. The residue was dissolved in saturated aqueous sodium bicarbonate. The aqueous layer was extracted (EtOAc). The organic 
layer was washed with saturated aqueous sodium bicarbonate. The basic layers were combined, acidified with aqueous $\mathrm{HCl}(6 \mathrm{~N})$ until obtaining pH 2 and extracted (EtOAc). The organic layer was dried over sodium sulphate, filtered and concentrated under vacuum to afford the title compound $(19.7 \mathrm{~g}, 93 \%)$ as a colorless oil: ${ }^{1} \mathrm{H}$ NMR $\left(\mathrm{CDCl}_{3}, 500 \mathrm{MHz}\right) \delta 5.95-5.87(\mathrm{~m}, 1 \mathrm{H}), 4.58(\mathrm{~m}, 2 \mathrm{H}), 5.49(\mathrm{br} \mathrm{d}$, $J=7.5 \mathrm{~Hz}, 1 \mathrm{H}), 5.23-5.20(\mathrm{~m}, 1 \mathrm{H}), 5.32-5.29(\mathrm{~m}, 1 \mathrm{H}), 4.39-4.36(\mathrm{~m}$, $1 \mathrm{H}), 2.47-2.42(\mathrm{~m}, 2 \mathrm{H}), 2.23-2.17(\mathrm{~m}, 1 \mathrm{H}), 2.03-1.95(\mathrm{~m}, 1 \mathrm{H}), 1.44$ ( $\mathrm{s}, 9 \mathrm{H})$.

\subsection{2. tert-Butyl (S)-4-(((allyloxy)carbonyl)amino)-5-(methoxy-} (methyl)amino)-5-oxopentanoate

To a cooled solution of (S)-2-(((allyloxy)carbonyl)amino)-5(tert-butoxy)-5-oxopentanoic acid $(19.7 \mathrm{~g}, 68.5 \mathrm{mmol})$ in DCM $(390 \mathrm{~mL})$ were added $\mathrm{N}, \mathrm{O}$-dimethylhydroxylamine hydrochloride ( $8.02 \mathrm{~g}, 82.2 \mathrm{mmol})$, 4-methylmorpholine ( $9.04 \mathrm{~mL}, 82.2 \mathrm{mmol})$ and 1,(3-dimethylaminopropyl)-3-ethylcarbodiimide hydro-chloride $(15.7 \mathrm{~g}, 82.2 \mathrm{mmol})$. After $3 \mathrm{~h}$ of stirring at room temperature, the medium was washed with aqueous $\mathrm{HCl}(1 \mathrm{~N})$, with brine, dried over sodium sulphate, filtered and concentrated in vacuo. Purification of the residue by chromatography on silica gel (cyclohexane-EtOAc, gradient of $9 / 1$ to $6 / 4$ ) afforded the title compound ( $16.5 \mathrm{~g}, 72 \%$, as colorless oil): ${ }^{1} \mathrm{H}$ NMR $\left(\mathrm{CDCl}_{3}, 500 \mathrm{MHz}\right) \delta 5.86-5.93(\mathrm{~m}, 1 \mathrm{H}), 5.48$ (br d, $J=8.5 \mathrm{~Hz}, 1 \mathrm{H}), 5.31-5.27(\mathrm{~m}, 1 \mathrm{H}), 5.21-5.18(\mathrm{~m}, 1 \mathrm{H})$, 4.75-4.70 (m, 1H), 4.57-4.50 (m, 2H), $3.77(\mathrm{~s}, 3 \mathrm{H}), 3.20(\mathrm{~s}, 3 \mathrm{H}), 2.31$ $(\mathrm{t}, J=7.5 \mathrm{~Hz}, 2 \mathrm{H}), 2.06-2.00(\mathrm{~m}, 1 \mathrm{H}), 1.89-1.84(\mathrm{~m}, 1 \mathrm{H}), 1.42(\mathrm{~s}, 9 \mathrm{H})$.

\subsection{3. tert-Butyl (S)-4-(((allyloxy)carbonyl)amino)-5-} oxopentanoate

A solution of lithium aluminium hydride $(2 \mathrm{~N}$ in THF, $4.92 \mathrm{~mL}$, $9.8 \mathrm{mmol})$ was added dropwise to a cooled $\left(-78^{\circ} \mathrm{C}\right)$ solution of tert-butyl (S)-4-(((allyloxy)carbonyl)amino)-5-(methoxy-(methyl) amino)-5-oxopentanoate $(5.0 \mathrm{~g}, 15.1 \mathrm{mmol})$ in anhydrous THF $(67 \mathrm{~mL})$. The mixture was stirred at $-78^{\circ} \mathrm{C}$ for $3 \mathrm{~h} 30$, then aqueous $\mathrm{HCl}(1 \mathrm{~N})$ was slowly added to the medium, and the temperature was allowed to warm to $0^{\circ} \mathrm{C}$. The mixture was diluted with EtOAc. The aqueous layer was extracted (EtOAc, two times). The combined organic layer was washed with aqueous $\mathrm{HCl}(1 \mathrm{~N})$, with brine, dried over sodium sulphate, filtered and concentrated in vacuo to afford the title compound ( $4.27 \mathrm{~g}$, quantitative, as yellow oil): ${ }^{1} \mathrm{H}$ NMR $\left(\mathrm{CDCl}_{3}, 500 \mathrm{MHz}\right): \delta 9.59(\mathrm{~s}, 1 \mathrm{H}), 5.95-5.87(\mathrm{~m}, 1 \mathrm{H}), 5.50(\mathrm{br} \mathrm{s}, 1 \mathrm{H})$, $5.31(\mathrm{~d}, J=17 \mathrm{~Hz}, 1 \mathrm{H}), 5.23-5.21(\mathrm{~m}, 1 \mathrm{H}), 4.58(\mathrm{~d}, J=5.5 \mathrm{~Hz}, 2 \mathrm{H})$, $4.31-4.28(\mathrm{~m}, 1 \mathrm{H}), 2.40-2.26(\mathrm{~m}, 2 \mathrm{H}), 2.25-2.21(\mathrm{~m}, 1 \mathrm{H}), 1.93-1.86$ $(\mathrm{m}, 1 \mathrm{H}), 1.42(\mathrm{~s}, 9 \mathrm{H})$.

\subsection{4. tert-Butyl (S)-4-(((allyloxy)carbonyl)amino)-5,5-}

\section{diethoxypentanoate}

To a freshly prepared solution of tert-butyl (S)-4-(((allyloxy) carbonyl)amino)-5-oxopentanoate $(4.27 \mathrm{~g}, 15.7 \mathrm{mmol})$ in absolute ethanol $(25 \mathrm{~mL})$ were added, under Argon atmosphere, triethyl orthoformate ( $15.7 \mathrm{~mL}, 94.4 \mathrm{mmol}), p$-toluenesulfonic acid ( $81 \mathrm{mg}$, $0.47 \mathrm{mmol}$ ) and $3 \AA$ molecular sieves. After stirring at room temperature overnight, the mixture was filtered over a pad of Celite and rinsed with EtOH. The filtrate was concentrated under reduced pressure and co-evaporated with toluene (three times). Purification of the residue by flash chromatography on silica gel (cyclohexaneEtOAc, gradient $9 / 1$ to $8 / 2$ ) afforded the title compound $(4.40 \mathrm{~g}$, $81 \%$, as pale yellow oil ): $\mathrm{LCMS}\left(t_{\mathrm{R}}=3.63 \mathrm{~min}\right.$, purity $\left.=98 \%\right), \mathrm{ESI}^{+} \mathrm{m} /$ $z 300.1(\mathrm{M}-\mathrm{OEt})+\mathrm{H})^{+} ;{ }^{1} \mathrm{H}$ NMR $\left(500 \mathrm{MHz}, \mathrm{CDCl}_{3}\right) \delta 5.95-5.83(\mathrm{~m}$, $1 \mathrm{H}), 5.30(\mathrm{dq}, J=17.2 \mathrm{~Hz}$ and $1.6 \mathrm{~Hz}, 1 \mathrm{H}), 5.19(\mathrm{dq}, J=10.4 \mathrm{~Hz}$ and $1.4 \mathrm{~Hz}, 1 \mathrm{H}), 4.90(\mathrm{~d}, J=9.5 \mathrm{~Hz}, 1 \mathrm{H}), 4.55(\mathrm{~m}, 2 \mathrm{H}), 4.36(\mathrm{~d}, J=2.8 \mathrm{~Hz}$, $1 \mathrm{H}), 3.80-3.74(\mathrm{~m}, 1 \mathrm{H}), 3.73-3.66(\mathrm{~m}, 2 \mathrm{H}), 3.55-3.48(\mathrm{~m}, 2 \mathrm{H}), 2.30$ $(\mathrm{t}, J=7.5 \mathrm{~Hz}, 2 \mathrm{H}), 1.96-1.88(\mathrm{~m}, 1 \mathrm{H}), 1.73-1.68(\mathrm{~m}, 1 \mathrm{H}), 1.43(\mathrm{~s}, 9 \mathrm{H})$, $1.21(\mathrm{~m}, 6 \mathrm{H}) ;{ }^{13} \mathrm{C}$ NMR $\left(101 \mathrm{MHz}, \mathrm{DMSO}-d_{6}\right) \delta 172.03,155.88,133.85$, 116.59, 103.03, 79.41, 64.17, 62.79, 61.79, 52.19, 31.42, 27.75, 24.36,
15.26; $[\alpha]_{\mathrm{D}}^{20}=-25.5^{\circ}(\mathrm{c}=10 \mathrm{~g} / \mathrm{L}, \mathrm{EtOH}) ; \operatorname{HRMS}(\mathrm{M}+\mathrm{H})^{+}$calculated for $\mathrm{C}_{17} \mathrm{H}_{31} \mathrm{NO}_{6} 345.2151$; found $368.2083(\mathrm{M}+\mathrm{Na})^{+}, 713.4276$ $(2 \mathrm{M}+\mathrm{Na})^{+}, 244.1206$ cyclized warhead eg., $\left(\mathrm{M}\left(-\mathrm{OEt},-\mathrm{C}_{4} \mathrm{H}_{9}\right)+\mathrm{H}\right)^{+}$.

4.9.5. tert-Butyl (4S)-5,5-diethoxy-4-(2-(3-isopropyl-5-(2-methyl4-(naphthalen-2-ylamino)-benzamido)-2,6-dioxo-3,6-dihydropyrimidin-1(2H)-yl)propanamido)pentanoate

Step 1: To a stirred solution 2-(3-isopropyl-5-(2-methyl-4(naphthalen-2-ylamino)benzamido)-2,6-dioxo-3,6-

dihydropyrimidin-1(2H)-yl)propanoic acid $(130 \mathrm{mg}, 0.26 \mathrm{mmol}$, 1.00 eq.) and pentafluorophenol (52.6 mg, $0.29 \mathrm{mmol}, 1.10$ eq.) in a mixture of DCM $(2.6 \mathrm{~mL})$ and DMF $(1.3 \mathrm{~mL})$, was added 1-ethyl-3(3-dimethylaminopropyl)carbodiimide hydrochloride $(54.8 \mathrm{mg}$, $0.29 \mathrm{mmol}, 1.10$ eq.) and the reaction mixture was stirred at room temperature for $30 \mathrm{~min}$.

Step 2: In a separate flask, a solution of (R)-3allyloxycarbonylamino-5,5-diethoxy-pentanoic acid tert-butyl ester (135 mg, $0.39 \mathrm{mmol}, 1.50$ eq.) and 1,3-dimethylbarbituric acid $(64.9 \mathrm{mg}, 0.42 \mathrm{mmol}, 1.60$ eq.) in a mixture of DCM $(2.6 \mathrm{~mL})$ and DMF $(650 \mu \mathrm{L})$ was degassed and purged with nitrogen then tetrakis(triphenylphosphine)palladium(0) $(12.0 \mathrm{mg}, \quad 0.01 \mathrm{mmol}, \quad 0.04$ eq.) was added. The reaction mixture was stirred at r.t. for $30 \mathrm{~min}$.

Step 3: The solution from the Alloc deprotection containing tertbutyl $(R)$-3-amino-5,5-diethoxypentanoate was added dropwise to the activated ester formed in Step 1 and the resulting solution was stirred for $2 \mathrm{~h}$ at r.t. The reaction mixture was diluted with DCM $(30 \mathrm{~mL})$ and washed with a saturated aqueous solution of $\mathrm{NaHCO}_{3}$. The aqueous phase was washed with $\mathrm{DCM}(2 \times 10 \mathrm{~mL})$. The organic phases were combined, dried over $\mathrm{MgSO}_{4}$ and concentrated to dryness. The residue was purified by flash chromatography (silica gel, gradient of $0-50 \%$ EtOAc in heptane) to afford tert-butyl (4S)5,5-diethoxy-4-(2-(3-isopropyl-5-(2-methyl-4-(naphthalen-2ylamino)-benzamido)-2,6-dioxo-3,6-dihydropyrimidin-1(2H)-yl) propanamido)pentanoate $(160 \mathrm{mg}, 83 \%)$ as a beige solid: LCMS $\left(t_{\mathrm{R}}=1.44 \mathrm{~min}\right.$, purity $\left.=100 \%\right), \mathrm{ESI}^{+} \mathrm{m} / z 744.46(\mathrm{M}+\mathrm{H})^{+} ;{ }^{1} \mathrm{H} \mathrm{NMR}$ $\left(\right.$ DMSO$\left._{-} d_{6}\right) \delta 8.94(\mathrm{~d}, J=17.4 \mathrm{~Hz}, 1 \mathrm{H}), 8.77(\mathrm{~d}, J=4.5 \mathrm{~Hz}, 1 \mathrm{H})$, $7.77-7.94(\mathrm{~m}, 3 \mathrm{H}), 7.58-7.65(\mathrm{~m}, 2 \mathrm{H}), 7.55(\mathrm{dd}, J=8.5,1.8 \mathrm{~Hz}, 1 \mathrm{H})$, $7.48(\mathrm{t}, J=7.5 \mathrm{~Hz}, 1 \mathrm{H}), 7.38$ (ddd, $J=10.4,7.3,4.5 \mathrm{~Hz}, 2 \mathrm{H}), 7.03-7.16$ $(\mathrm{m}, 2 \mathrm{H}), 5.29(\mathrm{qd}, J=6.9,4.4 \mathrm{~Hz}, 1 \mathrm{H}), 4.55(\mathrm{ddd}, J=13.4,7.9,3.4 \mathrm{~Hz}$, $1 \mathrm{H}), 3.40-3.71(\mathrm{~m}, 4 \mathrm{H}), 2.50(\mathrm{~d}, J=1.9 \mathrm{~Hz}, 3 \mathrm{H}), 2.26-2.40(\mathrm{~m}, 2 \mathrm{H})$, $1.57-1.86(\mathrm{~m}, 2 \mathrm{H}), 1.49$ (dd, $J=17.7,5.9 \mathrm{~Hz}, 3 \mathrm{H}), 1.35-1.44(\mathrm{~s}, 9 \mathrm{H})$, $1.41(\mathrm{~m}, 6 \mathrm{H}), 1.06-1.22(\mathrm{~m}, 6 \mathrm{H})$.

4.9.6. $N-(3-(1-(((3 S)-2-H y d r o x y-6-o x o t e t r a h y d r o-2 H-p y r a n-3-y l)$ amino)-1-oxopropan-2-yl)-1-isopropyl-2,4-dioxo-1,2,3,4tetrahydropyrimidin-5-yl)-2-methyl-4-(naphthalen-2-ylamino) benzamide (39)

To a stirred solution of (S)-5,5-diethoxy-4-(2-\{3-isopropyl-5-[2methyl-4-(naphthalen-2-ylamino)-benzoylamino]-2,6-dioxo-3,6dihydro-2H-pyrimidin-1-yl\}-propionylamino)-pentanoic acid tertbutyl ester (105 mg, $0.14 \mathrm{mmol}, 1.00$ eq.) in DCM $(3.15 \mathrm{~mL}$ ) was added trifluoroacetic acid $(3.15 \mathrm{~mL}, 41.1 \mathrm{mmol})$ at $0{ }^{\circ} \mathrm{C}$ and the reaction mixture was stirred at room temperature for $2 \mathrm{~h}$. The reaction mixture was treated with a saturated aqueous solution of $\mathrm{NaHCO}_{3}$ (aq) to bring the pH to 5 and extracted with DCM $(2 \times 5 \mathrm{~mL})$. The organic phases were combined, dried over $\mathrm{MgSO}_{4}$ and concentrated to dryness. The crude product was purified by flash chromatography (silica gel, gradient of $0-10 \% \mathrm{MeOH}$ in DCM). After concentration of the fractions to dryness, the gum was triturated in EtOAc and the solid was collected and dried in a vacuum oven at $40^{\circ} \mathrm{C}$ to afford $\mathrm{N}$-(3-(1-(((3S)-2-hydroxy-6-oxotetrahydro2H-pyran-3-yl)amino)-1-oxopropan-2-yl)-1-isopropyl-2,4-dioxo1,2,3,4-tetrahydropyrimidin-5-yl)-2-methyl-4-(naphthalen-2-ylamino)benzamide $(32.0 \mathrm{mg}, 35 \%)$ as a pale pink solid: LCMS $\left(t_{\mathrm{R}}=5.57 \mathrm{~min}\right.$, purity $\left.=93 \%\right), \mathrm{ESI}^{+} \mathrm{m} / z 612.35(\mathrm{M}+\mathrm{H})^{+} ;{ }^{1} \mathrm{H} \mathrm{NMR}$ 
$\left(\right.$ DMSO- $\left.d_{6}\right) \delta 12.18(\mathrm{~s}, 1 \mathrm{H}), 9.40(\mathrm{~d}, J=17.4 \mathrm{~Hz}, 1 \mathrm{H}), 8.94(\mathrm{~d}, J=5.6 \mathrm{~Hz}$, $1 \mathrm{H}), 8.72(\mathrm{~s}, 1 \mathrm{H}), 8.42(\mathrm{~s}, 1 \mathrm{H}), 7.72-7.88(\mathrm{~m}, 3 \mathrm{H}), 7.58(\mathrm{~d}, J=2.2 \mathrm{~Hz}$, $1 \mathrm{H}), 7.50(\mathrm{~d}, J=8.5 \mathrm{~Hz}, 1 \mathrm{H}), 7.43(\mathrm{t}, J=7.5 \mathrm{~Hz}, 1 \mathrm{H}), 7.27-7.38(\mathrm{~m}$, $2 \mathrm{H}), 7.05$ (dd, $J=18.0,5.4 \mathrm{~Hz}, 2 \mathrm{H}), 5.40(\mathrm{p}, J=6.8 \mathrm{~Hz}, 1 \mathrm{H}), 4.78(\mathrm{td}$, $J=6.8,3.2 \mathrm{~Hz}, 1 \mathrm{H}), 2.44(\mathrm{~s}, 3 \mathrm{H}), 2.15-2.33(\mathrm{~m}, 2 \mathrm{H}), 1.96-2.02(\mathrm{~m}$, $1 \mathrm{H}), 1.77-1.60(\mathrm{~m}, 1 \mathrm{H}), 1.45(\mathrm{~d}, J=6.9 \mathrm{~Hz}, 3 \mathrm{H}), 1.33(\mathrm{q}, J=7.4,6.4 \mathrm{~Hz}$, $6 \mathrm{H}) ;{ }^{13} \mathrm{C}$ NMR $\left(101 \mathrm{MHz}\right.$, DMSO-d $\left.d_{6}\right) \delta 169.60,167.47,159.04,156.32$, 149.35, 145.38, 145.04, 140.15, 134.26, 131.60, 129.56, 128.99, 128.72, $127.50,126.52,126.40,123.46,120.60,118.21,113.60,111.53,112.81$, 100.54, 95.21, 58.72, 58.29, 58.04, 20.93, 20.69, 20.57, 13.95, 13.87; HRMS: $(\mathrm{M}+\mathrm{H})^{+}$calculated for $\mathrm{C}_{33} \mathrm{H}_{35} \mathrm{~N}_{5} \mathrm{O}_{7}$ 614.2536; found 614.2603.

\section{Acknowledgments}

The authors would like to acknowledge the support of our CRO partners, Spirochem, Santai Labs and Syngene.

\section{References}

[1] D.R. Mcllwain, T. Berger, T.W. Mak, Cold Spring Harb. Perspect. Biol. (2013) $1-28$.

[2] a) S.M. Tuchayi, E. Makrantonaki, R. Ganceviciene, C. Dessinioti, S.R. Feldman, C.C. Zouboulis, Nat. Rev. Disease Primers 1 (2015) 1-20; b) C. Dessinioti, A. Katsambas, Clin. Dermatol. 28 (2010) $2-7$.

[3] a) M. Kistowska, S. Gehrke, D. Jankovic, K. Kerl, A. Fettelschoss, L. Feldmeyer, G. Fenini, A. Kolios, A. Navarini, R. Ganceviciene, J. Schauber, E. Contassot, L.E. French, J. Invest. Dermatol. 134 (2014) 677-685;

b) E. Contassot, L.E. French, J. Invest. Dermatol. 134 (2014) 310-313.

[4] H.-L. Kelhälä, R. Palatsi, N. Fyhrquist, S. Lehtimäki, J.P. Väyrynen, M. Kallioinen, M.E. Kubin, D. Greco, K. Tasanen, H. Alenius, B. Bertino, I. Carlavan, B. Mehul, S. Déret, P. Reiniche, P. Martel, C. Marty, U. Blume-Peytavi, J.J. Voegel,
A. Lauerma, PLoS One 9 (2014) 1-18.

[5] a) T. O'Brien, S.D. Linton, Design of Caspase Inhibitors as Potential Clinica Agents, CRC Press/Taylor \& Francis Group, Boca Raton, 2009, pp. 82-132;

b) M. Poreba, A. Strozyk, G.S. Salvesen, M. Drag, Cold Spring Harb. Perspect. Biol. (2013) 1-20;

d) N.A. Thornberry, H.G. Bull, J.R. Calaycay, K.T. Chapman, A.D. Howard, M.J. Kostura, D.K. Miller, S.M. Molineaux, J.R. Weidner, J. Aunins, K.O. Elliston, J.M. Ayala, F.J. Casano, J. Chin, G.J.-F. Ding, L.A. Egger, E.P. Gaffney, G. Limjuco, O.C. Palyha, S.M. Raju, A.M. Rolando, J.P. Salley, T.-T. Yamin, T.D. Lee, J.E. Shively, M. MacCross, R.A. Mumford, J.A. Schmidt, M.J. Tocci, Nature 356 (1992) 768-774.

[6] a) B. Howley, H.O. Fearnhead, J. Cell Mol. Med. 12 (2008) 1502-1516; b) S.H. MacKenzie, J.L. Schipper, A.C. Clark, Curr. Opin. Drug Discov. Dev 13 (2010) 568-576

c) W. Wannamaker, R. Davies, M. Namchuk, J. Pollard, P. Ford, G. Ku, C. Decker, P. Charifson, P. Weber, U.A. Germann, K. Kuida, J.C. Randle, J. Pharmacol. Exp. Therapeut. 321 (2007) 509-516.

[7] J.-F. Fournier, L. Clary, S. Chambon, L. Dumais, C.S. Harris, C. Millois, R. Pierre, S. Talano, E. Thoreau, J. Aubert, M. Aurelly, C. Bouix-Peter, A. Brethon, L. Chantalat, O. Christin, G. El-Bazbouz, A.-L. Ghilin, T. Isabet, C. Lardy, A.P. Luzy, C. Mathieu, K. Mebrouk, D. Orifla, J. Pascau, K. Reverse, R. Roche, V. Rodeschini, L.F. Hennequin, J. Med. Chem. 61 (2018) 4030-4051.

[8] A. Brethon, C. Bouix-Peter, L. Clary, J.-F. Fournier, C.S. Harris, C. Lardy, D. Roche, V. Rodeschini, S. Talano, Tetrahedron Lett. 57 (2016) 5924-5927.

[9] L. Trottet, H. Maibach, Dermal Drug Selection and Development - an Industrial Perspective, Springer, 2017, pp. 29-48.

[10] a) R.-K. Chang, A. Raw, R. Lionberger, L. Yu, C.J. Mugglestonea, AAPS J. 15 (2013) 41-52;

b) S. Mariz, M.E. Lanec, Int. J. Pharm. 435 (2012) 22-26.

[11] J.-G. Boiteau, C. Bouix-Peter, S. Chambon, L. Clary, S. Daver, L. Dumais, J. F. Fournier, C.S. Harris, K. Mebrouk, C. Millois, R. Pierre, N. Rodeville, S. Talano, L. Tomas, Tetrahedron Lett. 57 (2016) 2367-2371.

[12] T. O'Brien, B.T. Fahr, M.M. Sopko, JW Lam, N.D, Waal, B.C Raimundo, H.E. Purkey, P. Pham, M.J. Romanowski, Acta Crystallogr. Sect. F Struct. Biol. Cryst. Commun. 61 (2005) 451-458.

[13] T.T. Talele, J. Med. Chem. 59 (2016) 8712-8756.

[14] K. Ramadas, N. Srinivasan, Synth. Commun. 22 (1992) 3189-3195.

[15] A. El-Faham, R. Subirós-Funosas, R. Prohens, F. Albericio, Chem. Eur J. 15 (2009) 9404-9416.

[16] Data supporting the chiral degradation study looking notably at the effect of changing excipients, temperature and $\mathrm{pH}$ is included in the supporting information.

[17] Wos, J. A.; Wang, Y.; Oppong, K. A.; O'Neill, S. V.; Laufersweiler, M. C.; Soper, D. L.; De, B.; Demuth, T. P. WO 2003103677. 